

\section{DISCLAIMER}

This document was prepared as an account of work sponsored by the United States Government. While this document is believed to contain correct information, neither the United States Government nor any agency thereof, nor The Regents of the University of California, nor any of their employees, makes any warranty, express or implied, or assumes any legal responsibility for the accuracy, completeness, or usefulness of any information, apparatus, product, or process disclosed, or represents that its use would not infringe privately owned rights. Reference herein to any specific commercial product, process, or service by its trade name, trademark, manufacturer, or otherwise, does not necessarily constitute or imply its endorsement, recommendation, or favoring by the United States Government or any agency thereof, or The Regents of the University of California. The views and opinions of authors expressed herein do not necessarily state or reflect those of the United States Government or any agency thereof, or The Regents of the University of California.

Ernest Orlando Lawrence Berkeley National Laboratory is an equal opportunity employer. 


\section{DISCLAIMER}

Portions of this document may be illegible in electronic image products. Images are produced from the best available original document. 


\title{
Ultrafast Studies of Electron Dynamics at Metal-Dielectric Interfaces
}

\author{
by \\ Nien-Hui Ge \\ Ph.D. Thesis \\ Department of Chemistry \\ University of California, Berkeley \\ and \\ Chemical Sciences Division \\ Lawrence Berkeley National Laboratory \\ University of California \\ Berkeley, California 94720
}

October, 1998

This work was supported by the Director, Office of Energy Research, Office of Basic Energy Sciences, Chemical Sciences Division, of the U.S. Department of Energy under Contract No. DE-AC03-76SF00098. 


\section{Ultrafast Studies of Electron Dynamics at}

\section{Metal-Dielectric Interfaces}

Copyright 1998

by

Nien-Hui Ge

The U.S. Department of Energy has the right to use this document for any purpose whatsoever, including the right to reproduce all or any part thereof. 


\begin{abstract}
by

Nien-Hui Ge

Doctor of Philosophy in Chemistry

University of California at Berkeley

Professor Charles B. Harris, Chair
\end{abstract}

Ultrafast Studies of Electron Dynamics at Metal-Dielectric Interfaces

Femtosecond time- and angle-resolved two-photon photoemission spectroscopy has been used to study fundamental aspects of excited electron dynamics at metaldielectric interfaces, including layer-by-layer evolution of electronic structure and twodimensional electron localization.

On bare $\mathrm{Ag}(111)$, the lifetimes of image states are dominated by their position with respect to the projected bulk band structure. The $n=2$ state has a shorter lifetime than the $n=1$ state due to degeneracy with the bulk conduction band. As the parallel momentum of the $n=1$ image electron increases, the lifetime decreases. With decreasing temperatures, the $n=1$ image electrons, with zero or nonzero parallel momentum, all become longer lived. 
Adsorption of one to three layers of n-heptane results in an approximately exponential increase in lifetime as a function of layer thickness. This results from the formation of a tunneling barrier through which the interfacial electrons must decay, consistent with the repulsive bulk electron affinity of n-alkanes. The lifetimes of the higher quantum states indicate that the presence of the monolayer significantly reduces coupling of the image states to the bulk band structure. These results are compared with predictions of a dielectric continuum model.

The study of electron lateral motion shows that optical excitation creates interfacial electrons in quasifree states for motion parallel to the n-heptane/ $\operatorname{Ag}(111)$ interface. These initially delocalized electrons decay into a localized state within a few hundred femtoseconds. The localized electrons then decay back to the metal by tunneling through the adlayer potential barrier. The localization time depends strongly on the electron's initial parallel momentum and exhibits a non-Arrhenius temperature dependence. The experimental findings are consistent with a 2-D selftrapping process in which electrons become localized by interacting with the topmost plane of the alkane layer. The energy dependence of the self-trapping rate has been modeled with an electron transfer theory. This analysis shows that self-trapping involves inter- and intramolecular vibrational modes of the overlayer and the nonArrhenius temperature dependence is a result of a strong quantum contribution from the intramolecular modes. These results for a model interface contribute to the fundamental understanding of electron behavior at the interface between metals and 
molecular solids. 
To my parents

and

Yung-Ya 


\section{Contents}

List of Figures vi

List of Tables viii

1 Introduction 1

2 Image States on Bare Metal Surfaces 5

2.1 Hydrogenic Model . . . . . . . . . . . . . . . . . . . 6

2.2 Multiple Reflection Theory . . . . . . . . . . . . . . . . . 9

2.3 Effective Masses . . . . . . . . . . . . . . . . . . . . 15

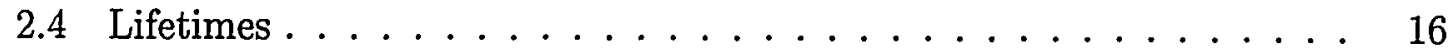

3 Experimental 22

3.1 Principles of Two-Photon Photoemission

3.2 High-Resolution Angle-Resolved TPPE

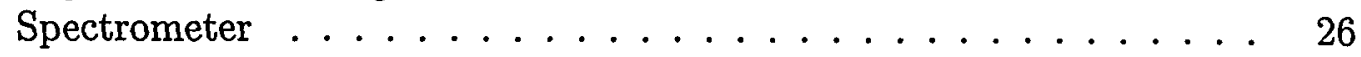

3.3 Extension to Femtosecond Time Resolution . . . . . . . . . . . 29

3.3.1 Pulse Compression ................. . . 29

3.3.2 Femtosecond Ti:Sapphire Laser System . . . . . . . . . . 36

3.4 Sample Preparation . . . . . . . . . . . . . . 40

4 Electron Dynamics on a Bare Ag(111) Surface 42

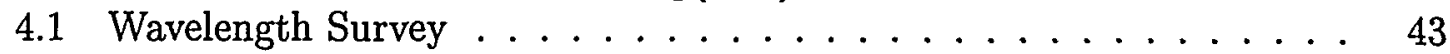

4.2 Dispersion Relation . . . . . . . . . . . . . . . . . . . . . . 49

4.3 Lifetime Measurements . . . . . . . . . . . . . . . 52

4.4 Temperature and Angle Dependence of Image State Dynamics . . . . 57

5 Layer-by-Layer Evolution of Electronic Structure 65

5.1 Previous Studies of Image Electrons at Metal-Alkane Interfaces . . . 67

5.2 Femtosecond Results for n-Alkanes/Ag(111) . . . . . . . . . 68 
5.3 Analysis and Discussion $\ldots \ldots \ldots \ldots \ldots \ldots \ldots$

5.4 Dielectric Continuum Model . . . . . . . . . . . . . . 77

5.5 Implications and Comparison to Electron Attractive Overlayers . . . . . . . . . . . . . . 86

6 Lateral Motion of the Interfacial Electrons: Dynamic Localization 91

6.1 Previous Studies with Picosecond Lasers . . . . . . . . . . . . . 92

6.2 Results of Femtosecond Time-Resolved Study . . . . . . . . . . . . 95

6.3 Survey of Possible Mechanisms . . . . . . . . . . . . . . . 100

6.4 Small Polaron Formation . . . . . . . . . . . . . . . . . . . . 106

6.5 Electron Transfer Theory . . . . . . . . . . . . . . . . . . . . 112

6.5.1 Spin-Boson Hamiltonian . . . . . . . . . . . . . . . . . 113

6.5.2 Rate Constant and Partition Function . . . . . . . . . . 116

6.5.3 Partition Function and Equivalent Ising Model . . . . . . . 120

6.5 .4 Limiting Cases . . . . . . . . . . . . . . . . . . . . . . 127

6.5 .5 Remarks . . . . . . . . . . . . . . . . 136

6.6 Analysis of Self-Trapping Dynamics . . . . . . . . . . . . . 137

6.7 Beyond the Single Quantum Mode Assumption . . . . . . . . . . . . . . . . . . . . 142

$\begin{array}{lll}7 \text { Conclusion } & 148\end{array}$

$\begin{array}{ll}\text { Bibliography } & 150\end{array}$ 


\section{List of Figures}

2.1 Hydrogenic model of image states . . . . . . . . . . . . 7

2.2 Schematic diagram for the multiple reflection theory . . . . . . . . . 11

2.3 Surface projected bulk band structure for $\operatorname{Ag}(111) \ldots \ldots \ldots \ldots$

3.1 Schematic diagram for angle-resolved two-photon photoemission . . . 23

3.2 High-resolution angle-resolved TPPE spectrometer $\ldots \ldots \ldots .28$

3.3 Schematic setup for a fiber-grating pulse compressor $\ldots \ldots \ldots 33$

3.4 Results of pulse compression . . . . . . . . . . . . . . 35

3.5 Femtosecond time- and angle-resolved TPPE spectrometer . . . . . 38

3.6 Autocorrelation of the laser pulses at $600 \mathrm{~nm} \ldots \ldots \ldots$. . . . . 39

4.1 TPPE kinetic energy (1-2.7 eV) spectra of $\mathrm{Ag}(111)$ at a series of wavelengths . . . . . . . . . . . . . . . . . . 44

4.2 Excitation scheme of bichromatic TPPE at a $\mathrm{Ag}(111)$ surface $\ldots .45$

4.3 TPPE kinetic energy $(2.5-5.8 \mathrm{eV})$ spectra of $\mathrm{Ag}(111)$ at a series of

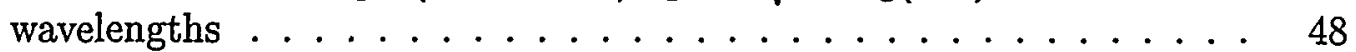

4.4 Angle-resolved TPPE studies of $\mathrm{Ag}(111) \ldots \ldots \ldots \ldots$

4.5 Dispersion data of the $n=1$ image state and the feature $D$ on $\operatorname{Ag}(111) 51$

4.6 TPPE kinetic energy spectra of $\mathrm{Ag}(111)$ at a series of pump-probe delays 53

4.7 The dynamics of the $n=1$ and surface states on $\operatorname{Ag}(111)$. The timedependence of the surface state serves as an instrument function for the time-resolution of our apparatus. . . . . . . . . . 54

4.8 The dynamics of the $n=1$ and 2 image states on $\operatorname{Ag}(111) \ldots \ldots$

4.9 TPPE spectra from $\mathrm{Ag}(111)$ for various temperatures . . . . . . 58

4.10 The TPPE peak positions of the $n=1$ image state and the surface state on $\mathrm{Ag}(111)$ as a function of temperature . . . . . . . . 59

4.11 Temperature and angle dependence of the $n=1$ image state dynamics on $\operatorname{Ag}(111) \ldots \ldots \ldots \ldots \ldots \ldots$ 
5.1 TPPE binding energy spectra for bare $\mathrm{Ag}(111)$, monolayer, and bilayer n-heptane $/ \operatorname{Ag}(111) \ldots \ldots \ldots . \ldots \ldots 96$

5.2 Dynamics of the $n=1,2$, and 3 states for monolayer n-heptane/ $\mathrm{Ag}(111) 70$

5.3 Population dynamics in the $n=1$ state for bare $\operatorname{Ag}(111)$, monolayer, and bilayer $n$-heptane $\operatorname{Ag}(111) \quad \ldots \ldots 73$

5.4 Model potential for bilayer n-octane/ $\operatorname{Ag}(111)$ calculated from the dielectric continuum model . . . . . . . . . . . . . . . . . 80

5.5 Experimental and calculated binding energies of the $n=1$ states as a function of n-octane coverage .............. 81

5.6 Probability density for the $n=1$ and 2 image states for bilayer noctane $/ \mathrm{Ag}(111)$ calculated from the dielectric continuum model . . .

6.1 TPPE processes and the projected bulk band structure for bilayer nheptane $/ \operatorname{Ag}(111) \ldots \ldots . \ldots . \ldots . . \ldots 96$

6.2 Femtosecond angle-resolved TPPE spectra for bilayer n-heptane/ $\mathrm{Ag}(111)$ taken at $120 \mathrm{~K}$ and a pump-probe delay time of $0 \mathrm{fs}$ and $1670 \mathrm{fs}$. . . $\quad 97$

6.3 Ultrafast time-resolved TPPE traces for delocalized and localized state for bilayer n-heptane on $\mathrm{Ag}(111)$ at various angles and $120 \mathrm{~K} \quad \ldots .98$

6.4 Temperature dependence of the dynamics of the delocalized and localized state for bilayer $n$-heptane $/ \mathrm{Ag}(111)$ at $16^{\circ}$. . . . . . . . . . . . 101

6.5 Temperature dependence of the dynamics of the delocalized state for bilayer $n$-heptane $/ \mathrm{Ag}(111)$ at various angles . . . . . . . . . . 104

6.6 Temperature dependence of the dynamics of the $n=2$ image state for bilayer $n$-heptane/Ag(111) at various angles . . . . . . . . . 105

6.7 Configuration coordinate diagram for self-trapping of an electron . . . 108

6.8 Free energy surfaces for the nonadiabatic and adiabatic limits . . . . 115

6.9 A model potential: $a q^{2}+b q^{4}$, where $a>0 \ldots \ldots$. . . . . . 117

6.10 Quantum path for the spin $\sigma_{n}(\tau) \ldots \ldots \ldots \ldots . \ldots \ldots$

6.11 Test of the electron transfer theory under the single quantum mode approximation .......................... 131

6.12 Effects of the strength of electron coupling on the electron transfer reaction rate in the normal and the inverted region . . . . . . 133

6.13 Oscillatory dependence of rate on the thermodynamic driving force . 135

6.14 Self-trapping rate as a function of exothermicity at $120 \mathrm{~K}$ and temperature dependence of self-trapping rate at $-\Delta \mathcal{E}=0.14 \mathrm{eV}$. Data and model analysis . . . . . . . . . . . . . . . 140

6.15 Self-trapping rate as a function of exothermicity at $50 \mathrm{~K} \ldots \ldots$ 
viii

\section{List of Tables}

4.1 Temperature and angle dependence of the $n=1$ image state dynamics

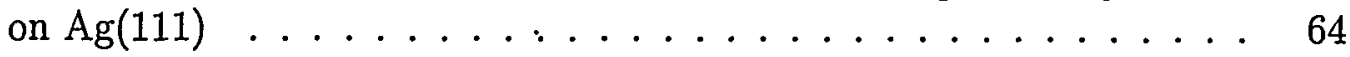

5.1 Comparison of measured image state lifetimes with values calculated from dielectric continuum model for lifetimes for $n$-heptane/ $\mathrm{Ag}(111)$

5.2 Experimental and calculated binding energies of the $n=1$ states as a function of n-octane coverage ............. 81 


\section{Acknowledgements}

I am grateful for having had so many wonderful people to work with during my years at Berkeley. I want to thank my advisor, Professor Charles Harris, for creating an exciting research environment, and providing me with the freedom to pursue my own ideas as well as the invaluable guidance and support at various pivotal points of my graduate career. Dr. Robert Lingle first brought me into the group, collaborated closely and fruitfully with me during his years at Berkeley, and he has since become a good friend and continued to provide me with advice and help. I have learned much from our discussions on subjects both scientific and theological. Robert Jordan taught me countless lessons in experiments. His patience and willingness to help younger students to grow make working with him and being his friend a very enjoyable experience. Jason McNeill has been a great source of ideas and help in data collection, analysis, and discussions because of his expertise and insight in experiment, theory, and computer. Chung Wong has been a masterful experimentalist as a coworker, and a good friend providing me with much laughter and occasional admonitions. Many of the data on localization would not have been possible without our joint efforts. Kelly Gaffney joined our team work during the later years of my graduate work, and has helped from doing experiments to beneficial discussions. The younger students Andre Miller and Simon Liu have also brought their share of enthusiasm to the surface projects and are pursuing some of the related questions raised in this work.

There have been many others in the group that I did not work with directly, but 
who supported me in many ways and made the group such an enjoyable working environment. Jennifer Hane, Steve Gadd, Jason King, Tim Lian, Steve Bromberg, Matt Asplund, Carsten Vala, Haw Yang, Ken Kotz, Matt Wilkens, and Preston Snee have been great members and I would like to thank all of them. Vijaya Narasimhn has been invaluable in the day-to-day operations of the group as well as always offering her encouragement.

I am grateful to Dr. Xueyu Song, former postdoctoral fellow in the Chandler group at Berkeley and now professor at Iowa State University, for always taking the time to discuss electron transfer theory with me. His input has been very helpful in the interpretation of data.

I have learned a great deal from my interaction with many Berkeley professors. Discussions with Professors William Miller, David Chandler, Steve Louie, Peter Yu, Yuen-Ron Shen, and Gabor Somorjai on my research has given me much inspiration. Dr. John Porter, former professor at Berkeley, was extremely helpful in teaching me the technique of electropolishing during my initial work here.

I want to thank my family for their love and support over all the years. My parents have always encouraged me in all I did and provided me with the best education possible. I can't thank them enough for all they have given me. My sisters, Suehui and Yichun, have been greatly supportive since we were little. I would like to thank my husband Yung-Ya. For almost eleven years we have gone through the challenge in college and graduate school together. I would not have been able to accomplish 
this work without his love and support.

This work was supported by the Director, Office of Energy Research, Office of Basic Energy Sciences, Chemical Sciences Division of the U. S. Department of Energy, under Contract No. DE-AC03-76SF00098. I would also like to acknowledge National Science Foundation support for specialized equipment used in some experiments described in this dissertation. 


\section{Chapter 1}

\section{Introduction}

The energy levels, transport properties, and dynamics of excited electrons at surfaces and interfaces are involved in many problems of fundamental interest and technological significance. For instance, hot photoexcited electrons at surfaces can induce electronic transition to dissociative states, thereby causing fragmentation and/or desorption of adsorbates [1]. Recent progress in organic light-emitting devices [2] has also heightened the demand for a deeper understanding of electronic processes at metalorganic interfaces, in which electron trapping, scattering, and the potential barrier at the interface can drastically affect carrier transport properties and the performance of devices. In order to develop a microscopic understanding of the behavior of electrons in complex media, a systematic study of the energy levels and dynamics of electrons associated with model interfaces is required.

The development of ultrahigh vacuum technology and surface preparation meth- 
ods over the past 30 years [3] has enabled researchers to grow ultrathin atomic and molecular adlayers on single crystal substrates. This offers an opportunity to investigate properties associated with Ångstrom scale materials in a well controlled fashion. To fully explore excited state dynamics of electrons at interfaces, experimental techniques must provide band-structure specificity, as well as time resolution. The conventional means of determining the band structure of excited surface electronic states is angle-resolved inverse photoemission spectroscopy [4]. This technique has been very successful, but has limited energy resolution and cannot be time-resolved. Scanning tunneling spectroscopy $[5,6]$ provides information on unoccupied energy levels with atomic resolution, but also has limited energy resolution and cannot provide time or momentum resolution. The method of two-photon photoemission (TPPE) $[7,8]$ has an order of magnitude better energy resolution than either of the aforementioned techniques and the ability to achieve time resolution when combined with ultrafast laser sources. TPPE is thus particular suited to study carrier dynamics at interfaces.

This dissertation investigates ultrafast dynamics of electrons at metal-dielectric interfaces with an emphasis on physisorbed systems. These studies were motivated by a desire to understand the following fundamental questions: Firstly, how do the dielectric properties and the electronic structure of the overlayer affect the interfacial potential? Secondly, how does the electron coupling to the metal substrate change as the dielectric layer increases in width in a layer-by-layer fashion? Lastly, electrons in metals are usually free-electron-like, but electrons in dielectric solids are usually 
localized. How does the transition of the electronic behavior occur at the interface and what do we expect the spatial extent of interfacial electrons to be?

To shed light on these questions, the electronic properties of interfaces have been explored from the vantage point of a unique type of surface electronic state called image states. It consists of an electron quantum-mechanically bound in the region of space just outside the material surface. As successive layers of adsorbates are grown on the surface, the evolution of the potential and the electronic structure of the interface can be studied by measuring the changes in the binding energy, effective mass, and lifetime in image state. TPPE offers exceedingly high resolution for studying such changes in these excited states at the interface.

This thesis is organized as follows. Relevant background information about image states on bare metal surfaces, including theoretical models and experimental findings is introduced in Chapter 2. Chapter 3 describes the experimental techniques used in this study. The extension of the original high-resolution angle-resolved TPPE spectrometer into the femtosecond time resolution by means of pulse compression constituted my initial work. This endeavor ended up in favor of Ti:sapphire laser systems which was utilized as the light source for the projects discussed in this thesis. Chapter 4 describes the first experiments done using our femtosecond TPPE spectrometer. The dynamics of image electrons on bare $\operatorname{Ag}(111)$ surface were investigated. This work established a scientific base to judge the significance of novel femtosecond results obtained on metal-dielectric systems. The results on physisorbed n-alkane/ $\operatorname{Ag}(111)$ 
interfaces is presented in Chapter 5 and 6 . Two aspects of the dynamics are discussed in detail. Firstly, the layer dependent electron binding energy and lifetime are analyzed and compared to a dielectric continuum model. These results show that the electron affinity of the overlayer material plays an important role in determining the electronic structure and dynamics. Secondly, by momentum-resolving the electron dynamics, the process of electron localization was investigated in real time. The data indicate that an initially delocalized electron at the interface forms a small polaron and a theoretical treatment yields information about the electron-lattice interaction energy and the lattice vibrational modes that mediate polaron formation. 


\section{Chapter 2}

\section{Image States on Bare Metal}

\section{Surfaces}

Electronic surface states play an important role in a wide variety of physical problems, affecting properties such as surface energy, chemical reactivity, film growth, surface conductivity, and adhesion. Most surface states arise from the loss of periodicity of the bulk, which results in states that are localized in the vicinity of the surface of a crystal $[9,10,11]$ Image states are a class of surface states that arises from the abrupt change of polarizability at an interface rather than from the loss of periodicity. An electron near the surface are bound by the Coulomb attraction to its image charge. In fact the Coulomb tail of the image potential allows an infinite Rydberg series of states to exist.

The existence of image states was first theoretical predicted $[12,13]$ and experi- 
mentally observed on the surface of liquid helium [14]. The first evidence for image potential states on bare metals was obtained by inverse photoemission $[15,16,17]$. Steinmann, Himpsel, and coworkers first studied this class of surface states by twophoton photoemission (TPPE) with nanosecond lasers [7]. This and subsequent TPPE work led to confirmation of the multiple reflection theory $[18,19]$ for image state binding energies on different single-crystal metal faces. In the recent years, however, the studies of image states in the presence of overlayers $[8,20]$ provided a new tool for characterizing and studying many important properties of interfaces on the nanometer scale.

In this chapter, some basic physics of image states on metals is elaborated since the interface electronic states discussed in the later chapters are derived from them. Reviews of this field has recently been given by Fauster and Steinmann [8] and Osgood and Wang [21].

\subsection{Hydrogenic Model}

For a bare metal, an electron in the vacuum is attracted to the polarization it induces in the surface (Fig. 2.1). This problem is often solved in elementary electrostatics by the method of images $[22,23]$, in which the electron at a distance $z$ from a surface interacts with a fictitious image charge of an opposite sign located at $-z$. This simple charge configuration mimics the boundary conditions for an electric field in the presence of a perfect conductor and thus provides the unique solution to 


\section{Surface \\ Polarization}
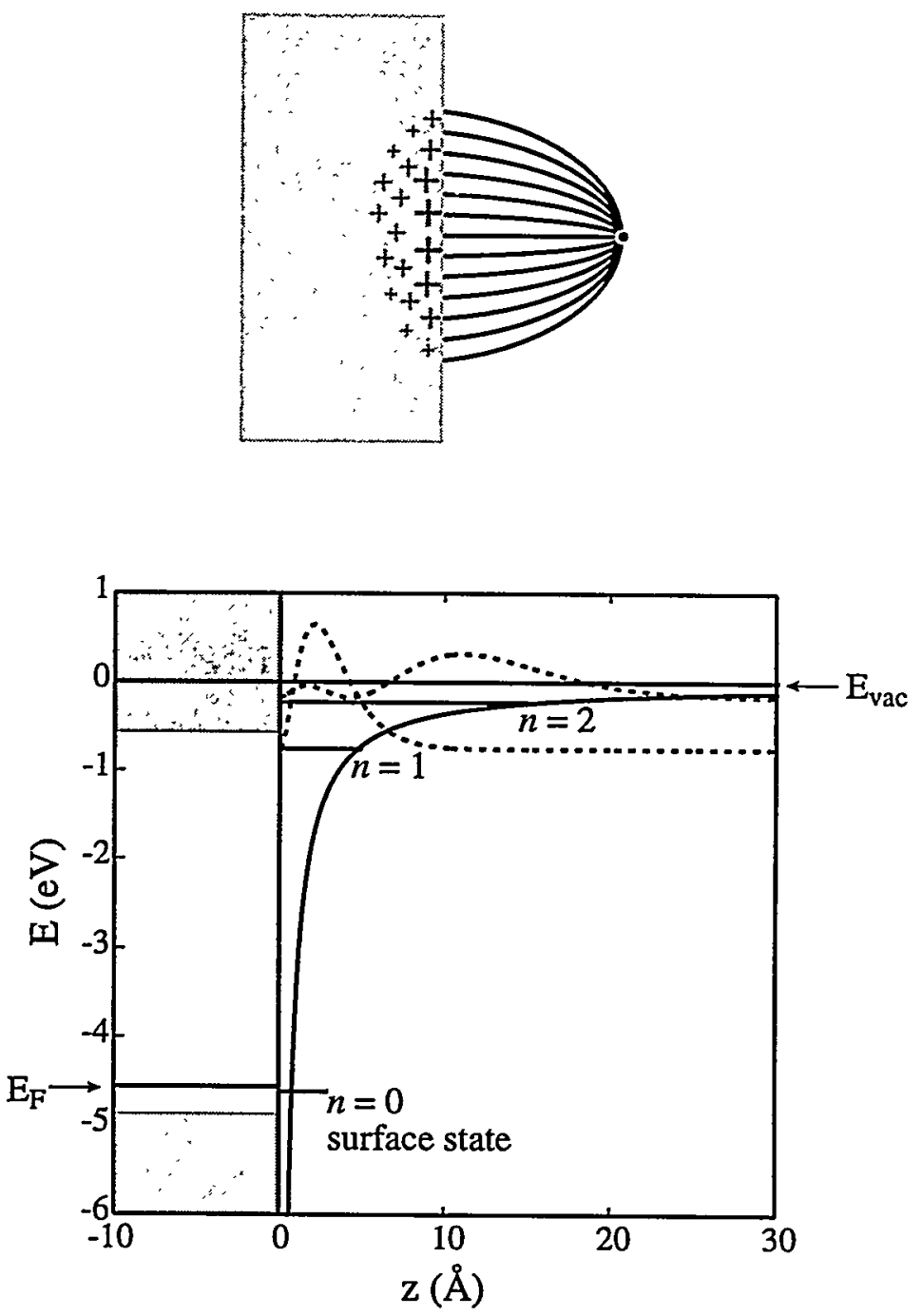

Figure 2.1: An electron near a surface is bound by an image potential which the electron induces by polarizing the material. Image states form a Rydberg series of states that converge to the vacuum level. The square of the hydrogenic wavefunction is shown for the lowest two states. The $\mathrm{Ag}(111)$ band structure is shown here for $k_{\|}=0$. 
Poisson's equation: a 1-D Coulomb potential

$$
V(z)=-\frac{e^{2}}{4 z} .
$$

For the idealized case of a completely reflective metal surface with a infinite high barrier, the situation corresponds to the hydrogen-atom problem in one dimension. The solution of the Schrödinger equation separates into a product of a 2-D wavefunction for free motion across the surface and a 1-D wavefunction of perpendicular motion:

$$
\Psi=\psi(z) \mathrm{e}^{\mathbf{i} \mathbf{k}_{\|} \cdot \mathbf{r}},
$$

and the total energy is the sum of the two eigenenergies

$$
E=E_{n}+\frac{\hbar^{2} k_{\|}^{2}}{2 m_{\mathrm{e}}}
$$

Here $m_{\mathrm{e}}$ is the free electron. The image potential in the $z$ direction results in the formation of a Rydberg series of image potential states converging to the vacuum level (Fig. 2.1) with binding energy

$$
E_{n}=-\frac{0.85}{n^{2}} \mathrm{eV}
$$


where $n=1,2, \ldots$ is the usual principal quantum number and the angular momentum is zero in this case. The wavefunction, $\psi(z)$, of an image state electron is equal to $z$ times the radial part of the wavefunction for the hydrogen-like atoms with an effective nuclear charge of $1 / 4$. The maximum of the wavefunction for the $n=1$ and 2 states is 2 and $11 \AA$ away from the surface.

\subsection{Multiple Reflection Theory}

The above simple picture of image states predicts a binding energy independent of metal substrates, a description contradicts the experimental findings. Real image potential states have a range of energies from $-0.533 \mathrm{eV}$ for $\mathrm{Ag}(100)$ to $-0.83 \mathrm{eV}$ for $\mathrm{Cu}(111)$ [8]. A more realistic and widely used treatment relating the metal band structure and the image potential to the image electron eigenenergies on bare metal surfaces emerges from the multiple reflection theory (MRT) $[24,18]$ or phase-shift analysis. The MRT formalism is simply a way to solve the matching condition between the exponentially decaying wave function in the substrate region and the hydrogenic wave function in the vacuum. Reviews on applications of MRT have been written by by Smith [25] and by Echenique and Pendry [26].

In this model, the image potential state is represented as a plane wave that propagates between the perfectly reflecting barrier of the metal crystal band gap and the image potential in the vacuum (Fig. 2.2 on page 11). Bound states occur when the 
total accumulated phase is an integral multiple of $2 \pi$ :

$$
\phi_{c}+\phi_{b}=2 \pi n
$$

Here $\phi_{c}$ and $\phi_{b}$ are the phase shifts accumulated in reflection from the crystal boundary and from the image potential barrier, respectively. The problem of calculating image state eigenenergies reduces to that of obtaining expressions for $\phi_{c}$ and $\phi_{b}$ as a function of energy. The eigenenergies are then the energy roots of Eqn. 2.5.

For a pure image potential, the barrier phase change may be written as $[24,27]$

$$
\phi_{b}=\left[\left(\frac{3.4 \mathrm{eV}}{E}\right)^{1 / 2}-1\right] \pi
$$

where $E$ is the electron energy with respect to the vacuum level. If $\phi_{c}$ can be treated as constant over the range of the Rydberg series, by inserting Eqn. 2.6 into Eqn 2.5, one finds the electron biuding energy takes the form of [24]

$$
\begin{aligned}
& E_{n}=-\frac{0.85 \mathrm{eV}}{(n+a)^{2}}, \\
& a=\frac{1}{2}\left(1-\frac{\phi_{c}}{\pi}\right)
\end{aligned}
$$

where $a$ is called the quantum defect parameter.

Clearly, the quantum defect depends on the crystal phase shift $\phi_{c}$, so is the binding energy of the image electrons. For the infinite-crystal-barrier case, $\phi_{c}=\pi$ and $a=0$, 


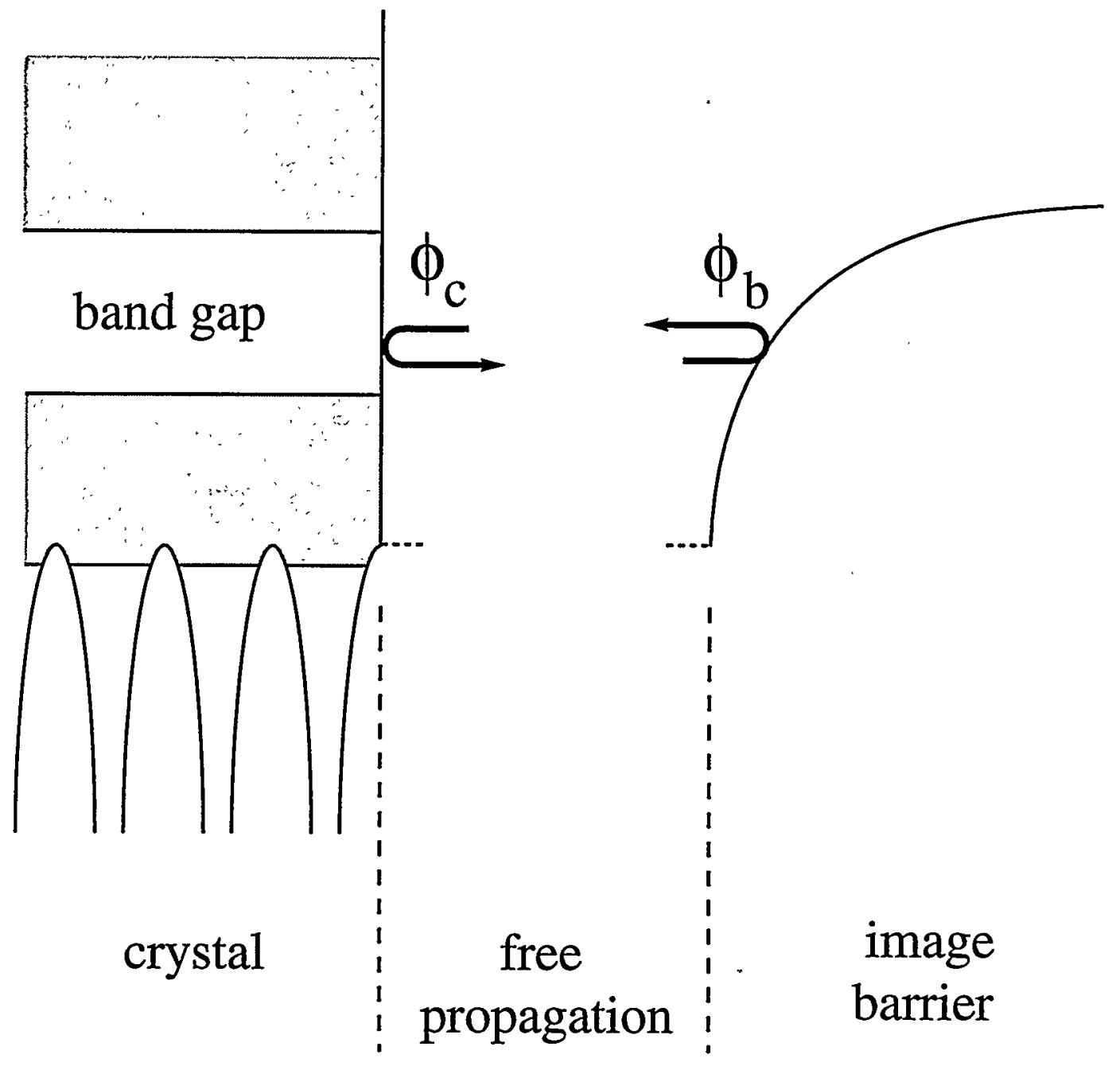

Figure 2.2: Schematic of electron scattering between between the crystal plane and the image potential barrier. 
the hydrogenic model is obtained. For certain crystallographic faces of various metals, $\phi_{c}$ depends on the the energetic position of the vacuum level, and hence the image potential states, relative to the projected bulk band structure [28]. Many metals exhibit a Shockley-inverted or s-p gap, where the gap is p-like at the bottom and s-like at the top, then $\phi_{c}$ varies from 0 at the bottom to $\pi$ at the top of the gap. Therefore, depending on the alignment of the vacuum level with the crystal band gap, the quantum defect can vary from 0 to 0.5 , leading to a decrease in binding energy going from the top to the bottom of the band gap.

An explicit account of the variation of $\phi_{c}$ with $E$ has been derived [19] with the metal band structure being described by the nearly-free-electron two-band model $[10,29]$. In this model, the crystal potential of the ion core is expanded in a Fourier series in multiples of the reciprocal lattice vector $\mathbf{g}$ by the following:

$$
V(z)=\sum_{m} V_{m \mathrm{~g}} \mathrm{e}^{\mathrm{i} m \mathrm{~g} \cdot \mathrm{r}}
$$

Keeping only the first two terms $V_{\mathrm{g}}, V_{-\mathrm{g}}$, the electron energies $\varepsilon$ with respect to the inner crystal potential for the two-band NFE model are given by solving the secular equation

$$
\left|\begin{array}{cc}
\left(\hbar^{2} / 2 m^{*}\right) \mathbf{k}^{2}-\varepsilon & V_{\mathbf{g}} \\
V_{\mathbf{g}} & \left(\hbar^{2} / 2 m^{*}\right)(\mathbf{k}-\mathbf{g})^{2}-\varepsilon
\end{array}\right|=0
$$


where $V_{\mathrm{g}}=V_{-\mathrm{g}}$ for crystals with inversion symmetry. At the zone boundary at $\mathrm{g} / 2$, there is an energy gap of width $2\left|V_{g}\right|$. Within the gap, the solutions corresponding to real $\varepsilon$ still exist, provided the wave vector $k_{z}$ is set to a complex quantity with the real and imaginary parts equal to $p$ and $q$, respectively. The imaginary part $q$ causes the wave to decay exponentially into the metal. For the case that $\mathrm{g}$ is along the surface normal $z$, Eqn 2.10 on the preceding page yields the standard results [19]

$$
\begin{aligned}
p & =g_{z} / 2 \\
\left(\hbar^{2} / 2 m^{*}\right) q^{2} & =\left(4 \varepsilon E_{g}+V_{g}^{2}\right)^{1 / 2}-\left(\varepsilon+E_{g}\right) \\
E_{g} & =\left(\hbar^{2} / 2 m^{*}\right) p^{2} \\
\sin (2 \delta) & =-\left(\hbar^{2} / 2 m^{*}\right)\left(p q / V_{g}\right)
\end{aligned}
$$

where $E_{g}$ is the midgap energy, and $m^{*}$ is the effective mass along the surface normal. The wavefunction in the crystal is then given as

$$
\psi=e^{q z} \cos (p z+\delta)
$$

This solution for the crystal substrate corresponds to an evanescent wave decaying into the metal substrate in the metal band gap. For a Shockley-inverted band gap and taking the origin of coordinates on a surface atom, this case corresponds to $V_{g}>0$. On the $z>z_{0}$ side of the terminating surface plane, consider a wavefunction of the 
the following form based on the idea of MRT:

$$
\psi=\mathrm{e}^{-\mathrm{i} \kappa z}+\phi_{c} \mathrm{e}^{\mathrm{i} \kappa z}
$$

where $\kappa$ is the wave vector of the plane wave. Matching the wavefunction of Eqn. 2.15 on the preceding page to that of Eqn. 2.16, one obtains

$$
\phi_{c}=2 \tan ^{-1}\left[\frac{p}{\kappa} \tan \left(p z_{0}+\delta\right)+\frac{q}{\kappa}\right] .
$$

Having the explicit $E$ dependence of $\phi_{b}$ and $\phi_{c}$ (Eqn. 2.6 on page 10 and Eqn. 2.17), the energies of the image states can be solved from Eqn. 2.5 on page 10. In fact, in the case of a Shockley-inverted gap and on an (111) surface, this theory also accounts for the existence of an additional $(n=0)$ surface state lying at an energy well below the lowest state $(n=1)$ of the image-potential Rydberg series. The wavefunction matching calculation determines the location of the maximum of the wavefunction in the image potential. For image states located near the top of a gap and $\phi_{c}$ is close to $\pi$, the maximum of the wavefunction is closer to the surface and resides in the steep part of the Coulomb potential well. Thus, the image electron will be more bound. Also, penetration of the evanescent wave into the metal should be maximum for states near the top of the gap and minimum for those near the center. This behavior has been discussed by Fauster [30] ${ }^{1}$ as well as in Ref $[8,21]$. This implies that, all other

\footnotetext{
${ }^{1}$ In this approach, a 1-D scattering model is employed. The complex band structure obtained is
} 
factors being equal, an $n=1$ image potential state near the top of the gap will overlap more strongly with bulk states and thus its lifetime would be shorter than one closer to the center.

\subsection{Effective Masses}

While the image states are localized perpendicular to the surface, parallel to the surface the electrons are delocalized and display free-electron-like dispersion (Eqn. 2.3 on page 8 ) if the crystal potential has no variation in the surface plane. In reality, the metal project bulk band structure does influence the lateral motion of the image electron. This effect can be taken into account by MRT through the dependence of $\phi_{c}$ and $\phi_{b}$ on $k_{\|}[26]$. Equation 2.5 on page 10 becomes $\phi_{c}\left(E, k_{\|}\right)+\phi_{b}\left(E, k_{\|}\right)=2 \pi n$. Expanding the equation to first order in $k_{\|}^{2}$ gives

$$
\begin{gathered}
E=E_{n}\left(k_{\|}=0\right)+\frac{\hbar^{2} k_{\|}^{2}}{2 m^{*}}, \\
m^{*}=\frac{\hbar^{2}}{d^{2} / d k_{\|}^{2}},
\end{gathered}
$$

where the deviation of the effective mass, $m^{*}$, from the free electron mass, $m_{\mathrm{e}}$, is a measure of the lateral variation of the surface potential. For image states lies near the middle of the band gap, theoretical predictions $[26,31]$ and extensive experimental studies on various bare metal surfaces $[8,31,32]$ found that $m^{*} \approx m_{\mathrm{e}}$. On the other

almost identical to the solution of the two-band model. 
hand, the effect the metal surface projected band structure on $m^{*}$ is clearly seen on the (111) surface where the $n=0$ surface state and $n=1$ image state are close to the bottom and the top of the band gap (Fig. 2.3). The width of the projected gap shrinks on moving away from the zone center $\bar{\Gamma}$ at $k_{\|}=0$. The lower edge of the gap disperses more rapidly than free-electron-like, while the upper edge is relatively flat. These characteristics are shared respectively by the $n=0$ and $n=1$ state with an effective mass intermediate between that of a free electron and the projected bulk band edge that the state crosses [19]. A geometrical construction based on the band gap dispersion [31] satisfactorily explains the experimentally observed effective masses, such as $m^{*}=1.3 m_{\mathrm{e}}$ for $\mathrm{Ag}(111)$ [31].

\subsection{Lifetimes}

The lifetime of the image electrons depends mainly on the electron's ability to couple to the substrate empty band structure. For typical metals, the Auger excitation of bulk electron-hole pairs provides the dominant relaxation process [33]. Because the wavefunctions are largely outside the metal surface, image electrons can be significantly longer-lived than electronic excitations in the metal [34,35]. This property makes them an ideal probe of the metal surface potential.

Theoretically, the decay of the image states can be modeled by introducing an imaginary component $V_{\mathrm{oi}}$ in the surface potential. Following Echenique and Pendry 


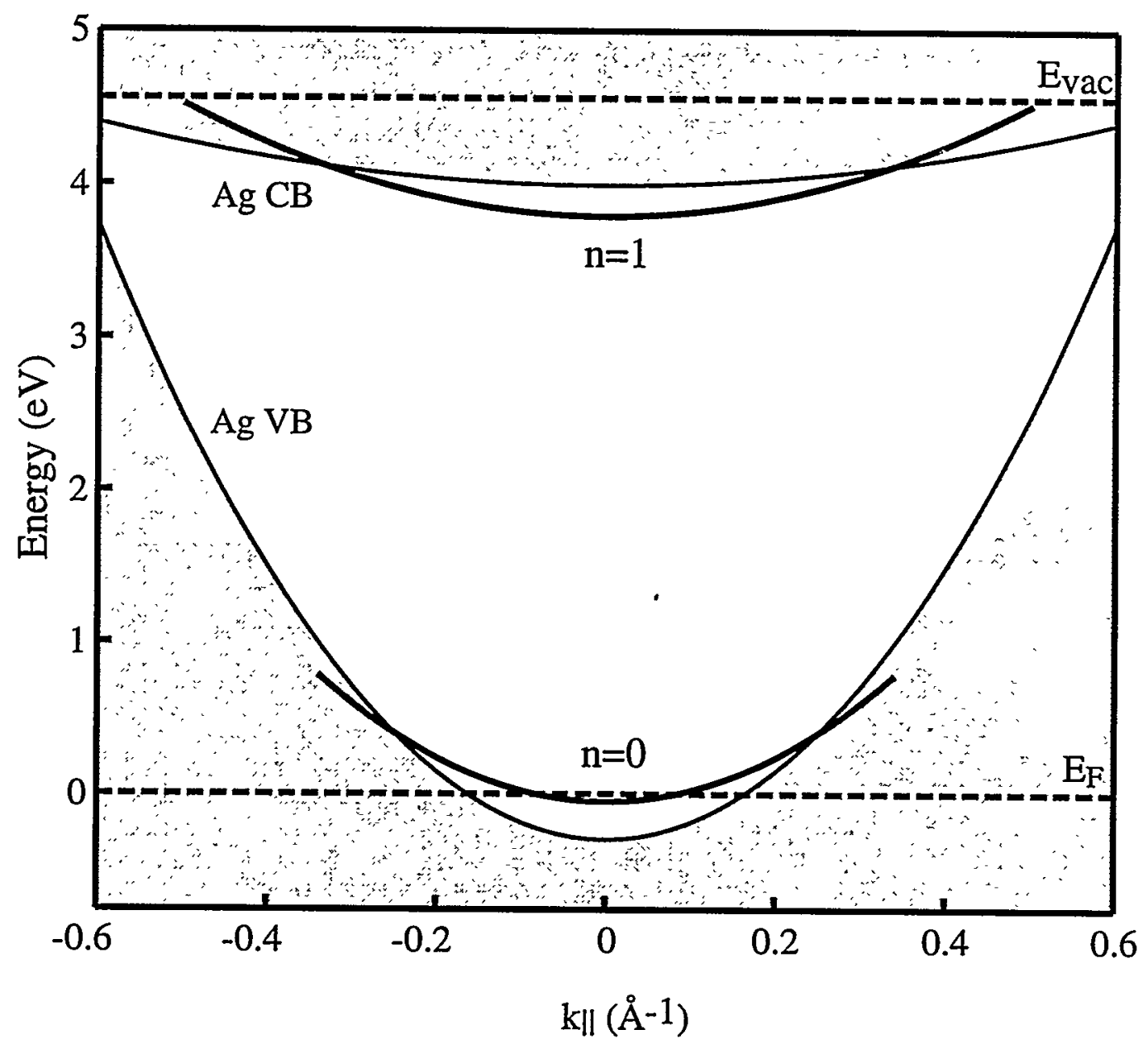

Figure 2.3: Surface projected bulk band structure for $\operatorname{Ag}(111)$. The $n=0$ and $n=1$ surface states are also displayed. 
[18], the energy width of the image state can be written as

$$
\frac{1}{2 \tau}=\frac{-V_{\mathrm{o}} \frac{\partial \phi_{c}}{\partial E}}{\frac{\partial}{\partial E}\left(\phi_{c}+\phi_{b}\right)},
$$

where $\tau$ is the image state lifetime. From Eqn. 2.5 on page 10 and Eqn. 2.7 on page 10 we know that

$$
\begin{aligned}
\frac{\partial}{\partial E}\left(\phi_{c}+\phi_{b}\right) & =2 \pi \frac{\partial n}{\partial E} \\
& =\frac{\pi}{0.85 \mathrm{eV}}(n+a)^{3}
\end{aligned}
$$

Therefore, the lifetime has a $(n+a)^{3}$ dependence, varying as $n^{3}$ for large $n$ and somewhat less strongly for small $n$, provided that the entire image Rydberg series is located in the projected bulk band gap and $\phi_{c}$ is more or less a constant over this energy range. This dependence on $n$ is a result of the decreasing spatial overlap with the bulk $[18,36]$ and has been confirmed by the first time-resolved TPPE experiments on $\operatorname{Ag}(100)[37,38,39]$ and a recent study using time-resolved TPPE in combination with the coherent excitation of several higher quantum states on $\mathrm{Cu}(100)$ [40].

A series of papers by Echenique and co-workers $[33,41,36]$ provide quantitative evaluation of the lifetime of image states within the GW approximation of many-body theory [42]. The lifetimes are evaluated from the knowledge of the imaginary part of the electron self-energy of the excited quasiparticle. Several important trends were drawn from these calculations. First, the calculation included both the interband 
and intraband decay processes. The interband relaxation concerns the decay of the image state with $k_{\|}=0$ into empty bulk states via excitation of some quasiparticles, mainly an electron-hole pair excitation. When the image state has nonzero $k_{\|}$, an additional contribution to lifetime shortening arises from intraband momentum scattering in which the electron decays into another image state with different $k_{\|}$. This extra linewidth broadening has been predicted to vary as $k_{\|}^{4}[33]$ when the scattering mechanism is a bulk Auger process. Second, the half-width of the image state depends strongly on the penetration of the wavefunction into the metal. Practically speaking, they exhibit a proportional relation. This effect is more important for the (111) noble-metal surfaces, where penetration of the wavefunction can not be realistically neglected like on the (100) surfaces [41]. Third, they found that the $n^{3}$-dependence of lifetime is quickly obtained beyond $n>5$ and confirmed the prediction in Ref. [18] that all members in the Rydberg series could be resolved with enough experimental resolution [36].

Although the penetration of the image state wavefunction into the bulk metal plays a very important role in determining the image state lifetime, one should not predict the lifetime behavior on two different crystallographic surfaces solely based on bulk penetration. For example, the $n=1$ image state lifetime on $\mathrm{Cu}(111)$ [43] is comparable to that on $\mathrm{Cu}(100)$ [40] but its wavefunction overlap with bulk is much larger on $\mathrm{Cu}(111)$ due to its close location to the top of band gap. Also, the existence of the $n=0$ surface state on $\mathrm{Cu}(111)$ provides an additional decay channel. In a 
recent lifetime calculation [44] it has been shown that these two factors that favor a faster decay are counterbalanced by the band gap on $\mathrm{Cu}(111)$ extending below the Fermi level, making the available phase space highly restricted.

The image potential always exists outside a metal surface, but quasi-stationary surface states result when their energies lie in a relative gap in the projected bulk band structure. This is the case for the entire Rydberg series for $\mathrm{Ag}(100), \mathrm{Cu}(100)$, and $\operatorname{Pd}(111)$, where the vacuum level is near the center of the gap. The higher quantum states $n \geq 2$ are degenerate with the bulk conduction band for $\mathrm{Ag}(111)$ and $\mathrm{Cu}(111)$ and they becomes surface resonances. These states can nevertheless be observed as relatively narrow features in TPPE spectroscopy. Apparently the effect on lifetime of degeneracy with the bulk conduction band is mitigated by the fact that the probability density for the higher quantum states is located farther out in the vacuum, reducing the coupling to the bulk states. Lifetime measurements on $\operatorname{Ag}(111)[39,45]$ and $\operatorname{Cu}(111)[43]$ have shown that the $n=2$ image potential state has a shorter lifetime than $n=1\left(\tau_{n=2}<\tau_{n=1} \leq 20 \mathrm{fs}\right)$, clearly opposite the usual trend of increasing lifetime with $n$. Here the relative placement of the image states with respect to the bulk band structure of the metal substrate, i.e. in or out of the gap, dominates the variation in image potential state lifetimes. The $n=2$ image states get broadened due to the direct coupling to energetically degenerated bulk band states. This elastic broadening has been calculated in Ref. [41].

The success of lifetime measurements on the bare surface suggests that lifetimes 
can be measured in more complex interfaces built on metal substrates, potentially involving a wide range of phenomena in nanometer-scale materials such as quantum confinement, electron tunneling, dielectric screening, localization, and magnetic ordering. Energy, effective mass, and lifetime measurements on image potential band structures at interfaces should yield detailed information about the potential near the interface, coupling across the adlayer, and the effective masses of carriers. This is the focus of the present work. 


\section{Chapter 3}

\section{Experimental}

\subsection{Principles of Two-Photon Photoemission Spectroscopy}

Unoccupied electronic structure and dynamics at interfaces can be studied by time- and angle-resolved TPPE. Angle-resolved photoemission is a well-established technique useful for determining band gaps and valence (occupied) band structure [46]. In general, photoemission requires the use of a photon whose energy is greater than the substrate work function, ejecting an electron from an occupied level below the Fermi energy. In TPPE $[7,47]$, one photon excites the electron from an occupied bulk or crystal-induced surface state into an unoccupied state (the state of interest) between the vacuum and Fermi levels; a second photon photoejects the electron into the vacuum for detection (Fig. 3.1A). The difference between the measured electron 
(A)

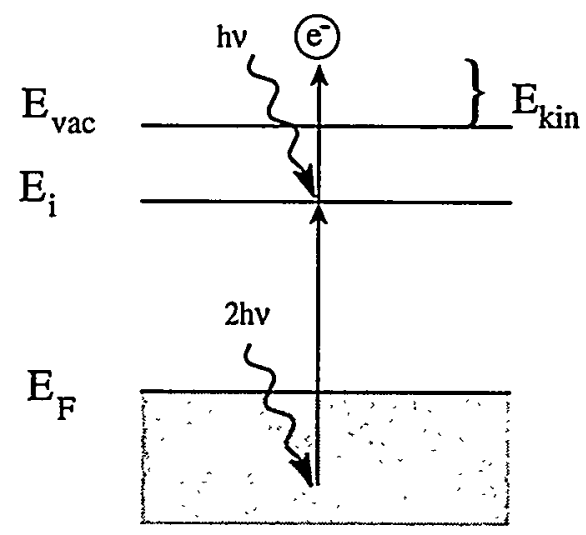

(B)
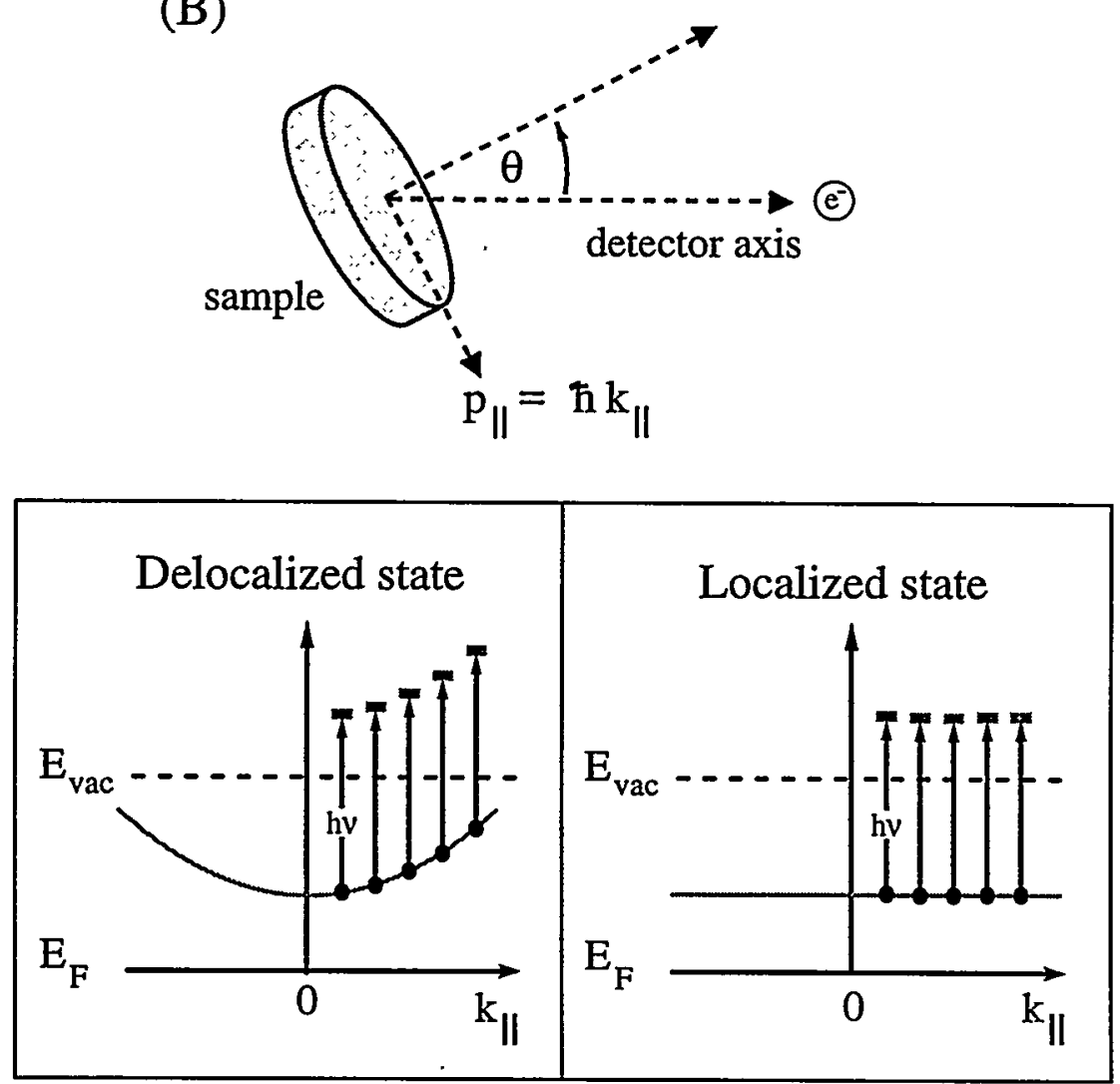

Figure 3.1: (A) Schematic energy diagram for two-photon photoemission (TPPE). (B) Schematic diagram for the photoejection process of angle-resolved TPPE. The dependence of photoelectron kinetic energy on parallel momentum $\hbar k_{\|}$is illustrated for delocalized and localized states. 
kinetic energy and the probe photon energy yields the binding energy of the intermediate state. TPPE thus differs from ordinary photoemission in that the kinetic energy of the photoemitted electron is determined by the photon energy and the binding energy of the intermediate state, rather than the initial state.

The interfacial band structure of the intermediate state can be mapped out by measuring the angle dependence of the photoelectron kinetic energy (Fig. 3.1B). For a well-ordered interface, the component of the electron wave vector parallel to the interface, $k_{\|}$, is conserved in the photoemission process [48]. Therefore, $k_{\|}$can be obtained from the kinetic energy, $E_{\text {kin }}$, of the photoemitted electron and the emission angles, $\theta$, with respect to the surface normal by

$$
k_{\|}=\left(2 m_{\mathrm{e}} E_{\mathrm{kin}} / \hbar^{2}\right)^{1 / 2} \sin \theta .
$$

For electrons that are delocalized parallel to the interface and behaving like free electron gas [49], the angle-resolved TPPE data will exhibit a dispersive band. The energy of these electrons is given by

$$
E=E_{0}+E_{\|}=E_{0}+\frac{\hbar^{2} k_{\|}^{2}}{2 m^{*}} .
$$

Here $k_{\|}$serves as a good quantum number and $E_{0}$ is the onset of the interfacial band at $k_{\|}=0$. The fact that the electrons are not truly free is accounted for through the use of an effective mass, $m^{*}$. The deviation of $m^{*}$ from $m_{\mathrm{e}}$ is a measure of the 
coupling of the electron with the bulk or an overlayer. Spatially localized electrons, on the other hand, result in non-dispersive features in the spectra. A localized state is a superposition of many $k_{\|}$plane waves; therefore photoelectrons can be ejected over a range of angles. The energy of the localized state, however, is independent of $k_{\|}$. Therefore, angle-resolved TPPE will yields a flat energy band as a function of angle.

In TPPE, the excited state dynamics can be time-resolved by varying the time delay between the first pump pulse and the second probe pulse, provided the laser pulse width is short enough. The decay of the photoelectron signal with increasing delay time is a direct measurement of the lifetime. Alternatively, the width of a peak in a TPPE spectrum could be used as an indication of the lifetime of the corresponding state, provided the energy resolution is good enough and the peak does not consist of several unresolved lines. The linewidth $\Gamma$ is related to the lifetime $\tau$ by $\Gamma \cdot \tau=\hbar=660 \mathrm{fs} \cdot \mathrm{meV}$.

The study of image potential states at metal surfaces using TPPE was pioneered by Steinmann and coworkers. Early work demonstrated the utility of TPPE for studying the lower members of the image potential Rydberg series outside metal surfaces $[7,31]$. A major contribution of their work was to systematically test the multiple reflection theory [18] for calculating the effect of metal band structure on the image potential state binding energies. Angle-resolved studies also demonstrated the feasibility of measuring the surface 2-D band structure [50]. Lifetime questions for a 
Chapter 3. Experimental

variety of bare metals and $\mathrm{O} / \mathrm{Ni}(100)$ have been approached through careful study of linewidths [51]. Their work on the study of metal-on-metal films [52, 53] and image states of reduced dimensionality on stepped metal surfaces [54] has been very fruitful. Direct measurement of image state lifetime on the $\operatorname{Ag}(111)$ and $\mathrm{Ag}(100)$ surfaces by using ultrafast TPPE was first done by Schoenlein et al. [37, 38, 39].

Recently, ultrafast TPPE techniques has been successfully applied to the studies of hot electron decay in metals $[55,56,35,57,58]$, carrier dynamics in semiconductors $[59,60,61]$, spin-dependent electron dynamics [62], coherent electron dynamics $[40,63]$, unoccupied quantum well lifetimes [64, 65], and electron localization [66]. Several excellent review articles regarding the development of TPPE and its versatile applications in a wide variety of systems have been published $[67,8,20,68,21]$

\subsection{High-Resolution Angle-Resolved TPPE}

\section{Spectrometer}

The initial work in the Harris laboratory on TPPE of electrons at interfaces $[69,70]$ was undertaken with the idea that high-repetition rate lasers coupled with time-offlight, single electron counting detection would minimize space charge broadening and give excellent energy resolution and signal-to-noise ratio. A schematic representation of the TPPE spectrometer that implemented this philosophy is shown in Fig. 3.2, and it has been described in detail previously $[70,71]$. A $1.9 \mathrm{MHz}$ train of 6 ps visible 
pulses were produced by a synchronously-pumped, cavity-dumped dye laser pumped by a mode-locked YLF laser. Ultraviolet pulses are generated by second harmonic generation in a $2 \mathrm{~mm} \beta$ barium borate (BBO) crystal. The second harmonic and the residual fundamental beam were focused colinearly on the sample with an incident angle of $45^{\circ}$. The polarization of the UV and visible pulses were usually set to be p-polarized. Electrons from the occupied bulk or surface states of the metal substrate are excited by a UV photon to an unoccupied image potential or other interface state. A visible photon then supplies the energy needed to photoemit the excited electron. Using two-color TPPE, it is possible to reduce the energy background and eliminate space charge broadening by reducing the intensity of the pump pulse and increasing the intensity of the probe pulse to get higher electron count rates [37]. The typical pulse energies were about $5 \mathrm{~nJ}$ in the UV and $50 \mathrm{~nJ}$ in the visible. The laser is usually tuned so that the photon energy of the UV is just below the work function in order to be able to investigate states near the vacuum while keeping one-photon photoemission to an acceptably low level.

The energy of the photoelectrons is determined by time-of-flight single-electron counting. The field-free flight region is $13.5 \mathrm{~cm}$ in length and is shielded by a cylinder of high-permeability alloy. A photodiode signal from the laser pulse provides the start pulse for time reference and a microchannel plate electron multiplier provides the stop pulse when it is struck by a photoelectron. The time difference is turned into a voltage by a time-to-amplitude converter and the voltage is recorded by a 


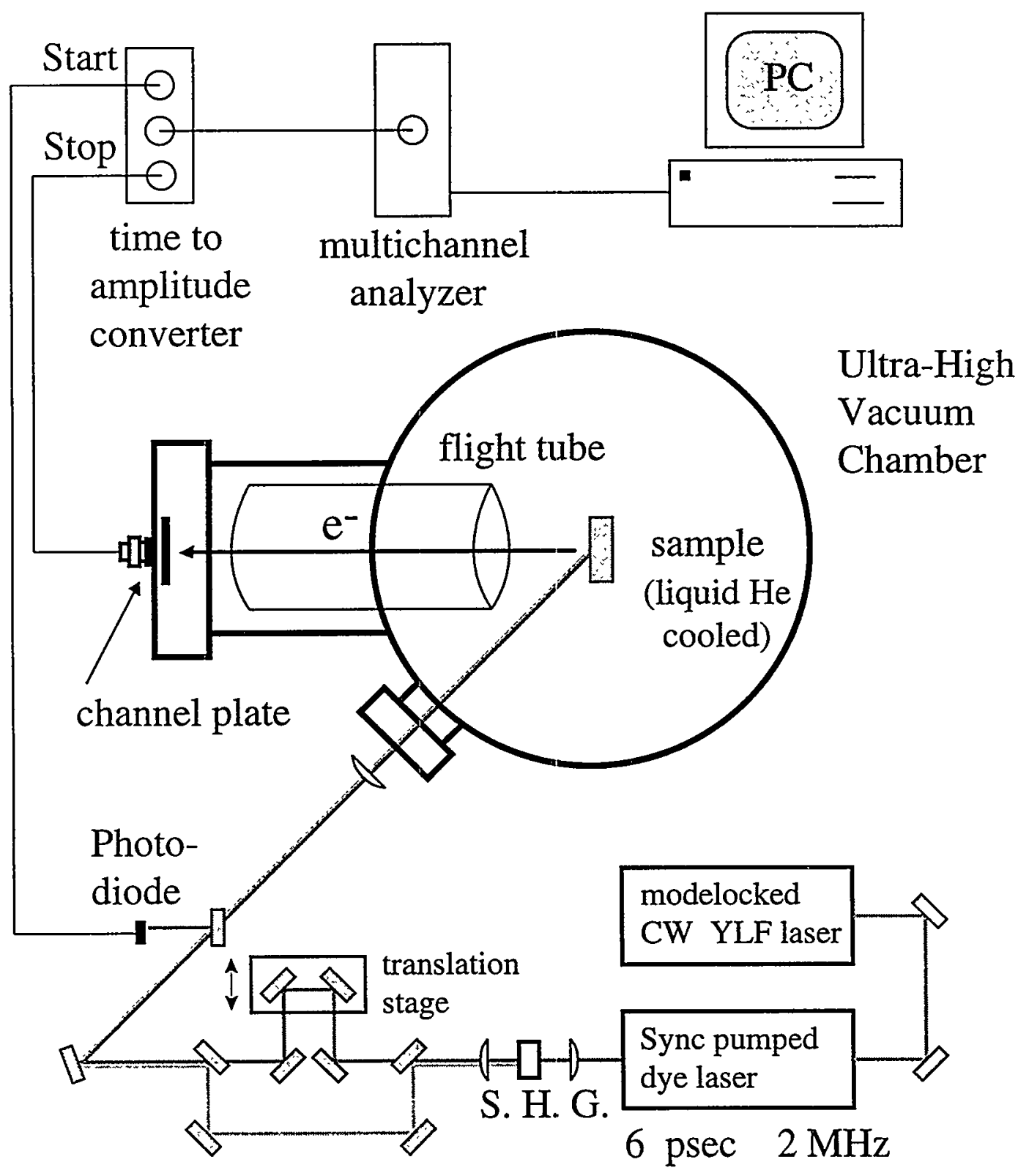

Figure 3.2: High-resolution angle-resolved TPPE spectrometer 
multichannel buffer. Even though only one photoelectron per hundred laser pulses is detected, high count rate is maintained due to the high repetition rate of the laser. The calculated instrumental energy resolution was $5 \mathrm{meV}$ or better at $1 \mathrm{eV}$ kinetic energy $[69,70,71]$. Image state features as narrow as $25 \mathrm{meV}$ FWHM were measured for monolayer $\mathrm{Xe} / \mathrm{Ag}(111)[70]$.

The parallel dispersion is measured by varying the angle of the sample with respect to the detector using a goniometer sector capable of angles between $-4^{\circ}$ and $20^{\circ}$. The angular acceptance of the detector is $\pm 1.5^{\circ}$. The wave vector parallel of the electron to the interface is determined form the sample angle and photoelectron kinetic energy

using Eqn. 3.1 on page 24. For electron kinetic energy of $2 \mathrm{eV}$, the $k_{\|}$resolution is $\Delta k_{\|} \approx 0.04 \AA^{-1}$. A fit to the parabolic dispersion relation, Eqn. 3.2 on page 24, determines the effective mass.

\subsection{Extension to Femtosecond Time Resolution}

\subsubsection{Pulse Compression}

A primary emphasis of my early graduate work was the extension of TPPE spectroscopy from the picosecond to the femtosecond time resolution. The original picosecond laser system was not capable of time-resolving the image state dynamics because the typical lifetime of image state electrons on bare metal surfaces is about tens to hundreds femtoseconds. To overcome this problem without setting up a new 
Chapter 3. Experimental

laser system, the method decided upon was to adapt the well-developed pulse compression technique to reduce our laser pulse width. The goal was to compress the pulse width down to $\sim 250 \mathrm{fs}$, generate the second harmonic, and use them directly to do the time-resolved experiment. This was a somewhat ambitious goal, since compressed pulses are commonly used to seed amplifiers, rather than being used directly in an experiment.

The fiber-grating pulse compression technique [72] employs optical fiber as the nonlinear medium to increase the bandwidth of laser pulses, and a grating pair as the dispersive delay line to rephase the spectral components. When a laser pulse is injected into a single-mode fiber with high enough intensity, the pulse experiences nonlinear as well as linear optical effects. In pulse compression, the self phase modulation (SPM) and the group velocity dispersion (GVD) are the two to consider. The index of refraction of the optical fiber is:

$$
n=n_{0}+n_{2} I(t),
$$

where $n_{0}$ is the linear or normal index, $n_{2}$ is the optical Kerr constant, i.e. the intensity-dependent term of the refractive index, and $I(t)$ is the intensity of the optical pulse. The time-variation of the pulse intensity leads to a variation in the index of refraction, and hence a modulation in the phase of the optical field. This phase modulation induced by the intensity variation of the optical pulse is called SPM. 
It gives rise to a shift in the instantaneous frequency, $\omega_{i}$, from the original carrier frequency, $\omega_{0}$, by an amount that depends on the magnitude of the change in intensity:

$$
\omega_{i}=\omega_{0}-\frac{2 \pi n_{2} L}{\lambda} \frac{d I(t)}{d t}
$$

where $L$ is the distance of propagation in the medium, and $\lambda$ is the original wavelength of the laser pulse. For the leading edge of the pulse, $\frac{d I(t)}{d t}>0, \omega_{i}$ is red-shifted. For the trailing edge, $\frac{d I(t)}{d t}<0, \omega_{i}$ is blue-shifted. By this SPM effect, new frequency components are generated.

In the visible spectrum, GVD is normal or positive. The red components of a pulse travel faster than the blue components. This broadens the temporal width of the pulse as the frequency components separate. The magnitude of this effect is directly proportional to the length of the fiber. As the intense laser pulse propagates through the fiber, it will be broadened in both the frequency and time domain.

A grating pair is then used to compress the pulse. The group delay time for a given frequency is determined by the optical path length for that frequency. The red component travels a longer path than the blue component This is precisely what is required to rephase the chirped output of the fiber, where the red components are on the leading edge of the pulse and the blue components are on the trailing edge. The separation between gratings controls the total phase delay and can be varied to match the spectrally-broadened pulse. In this regard, positive GVD can have an important 
effect on pulse compression. If a portion of the spectrally broadened pulse possesses a nonlinear chirp or no chirp at all, a pedestal or background will be present after compression by the grating pair. Strong SPM can combine with GVD to effectively linearize the frequency chirp over the entire pulse at the end of the fiber. This yields a compressed pulse with a clean shape and greatly reduced pedestal [73].

Figure 3.3 is a schematic of the experimental setup for pulse compression. The fiber is a polarization-preserving single mode fiber suitable for operation near $633 \mathrm{~nm}$. The delay line has double-pass configuration where the beam is diffracted four times. Thus the throughput is reduced. However, it produces a circular beam by canceling the transverse displacement of the different spectral components. The holographic grating has groove density of 1800 lines/mm and diffraction efficiency of $\sim 70 \%$ for $\mathrm{p}$ polarized light at $620 \mathrm{~nm}$ in the first order diffraction. A pair of microscopic objective lenses was used; one to focus the beam into one end of the fiber, and the other to collimate it at the other end. The polarization-preserving single mode fiber requires that the input beam is linearly polarized along the principle axis of the fiber, which is conveniently achieved by rotating the half-wave plate.

The parameters under control are the intensity and width of the input pulse, the length of fiber and the spacing between gratings. Using a procedure developed by Shank and coworkers $[74,75]$, quantities such as the achievable compression, the optimum fiber length, and the grating pair separation can be calculated. Consider the picosecond dye laser pulses to be compressed have the following characteristics: 


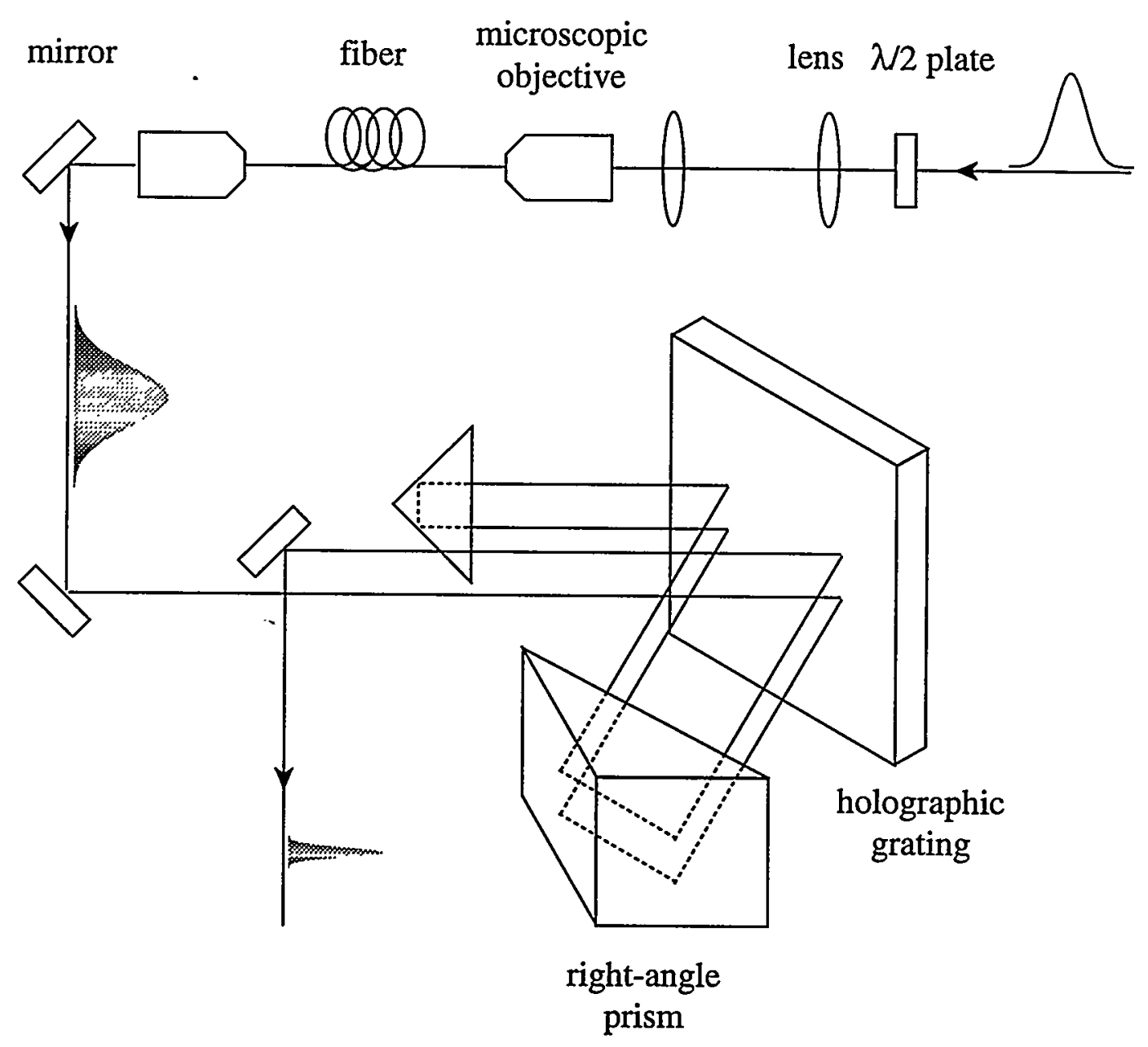

Figure 3.3: Schematic setup for a fiber-grating pulse compressor 
$\lambda=620 \mathrm{~nm}$, average power coupling into the fiber $\sim 40 \mathrm{~mW}$ at $1.9 \mathrm{MHz}$, autocorrelation FWHM $\sim 8$ ps. The optical fiber has a mode-field diameter $3.3 \mu \mathrm{m}$. The diffraction grating has groove density of 1800 lines/mm and the angle of diffraction is $\sim 35^{\circ}$. The calculation gave the following estimation: the achievable compression of $\sim 117$ corresponds to the compressed pulse of $\sim 68 \mathrm{fs}$, the optimum fiber length is $\sim 4.7 \mathrm{~m}$, and the proper separation between the grating pair is $\sim 18 \mathrm{~cm}$. Because this procedure may overestimate or underestimate some of the parameters, we systematically varied all of the parameters in order to achieve the best compression.

Our evaluation of this technique is summarized in the two sets of results shown in Fig. 3.4. The best results were obtained with a fiber length of 1-2 m. When the pump YLF laser was run at the high power (2W) and the output power at the end of fiber is $\sim 30-40 \mathrm{~mW}$, the broaden dye laser pulses can not be cleanly compressed. The autocorrelation of the compressed pulse showed a large pedestal (Fig. 3.4A) and the pulse width of $1.2 \mathrm{ps}$ was much longer than our goal. For an ideal input laser pulse where the intensity changes in the leading and trailing edges are sharp and symmetric, the frequency sweep produced by SPM should be symmetric around the original carrier frequency. The typical frequency spectrum at high power had a large spike at the original frequency and was very asymmetric. This indicates that a large fraction of the pulse did not undergo any SPM in the fiber and there must be some strange structure in the input pulse. Efforts were made to circumvent this problem, such as trying various power levels and changing the cavity length of the dye laser to 
(A)
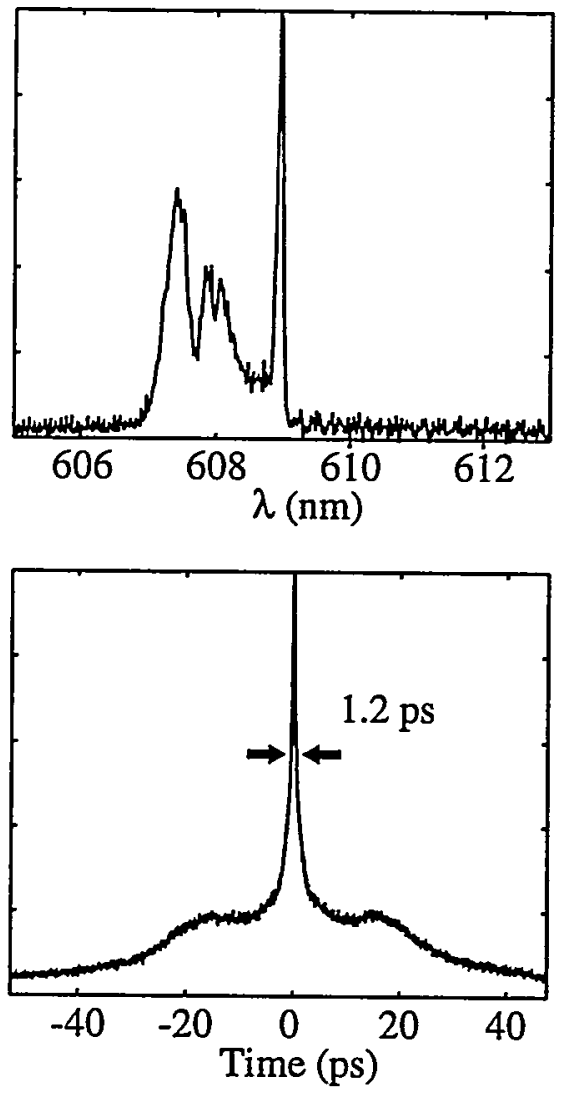

(B)
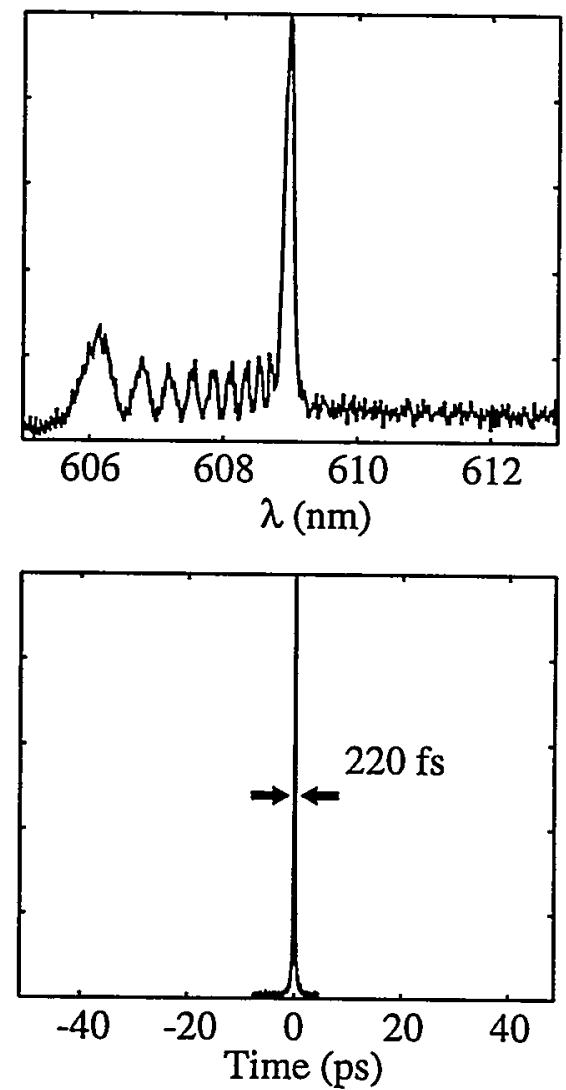

Figure 3.4: Results of pulse compression obtained at (A) high laser power and (B) low laser power. Top: the frequency spectrum of the laser pulse at the end of fiber. Bottom: autocorrelation trace of the laser pulse after rephasing by the grating pair. 
Chapter 3. Experimental

vary the pulse shape, but it turned out to be an insurmountable obstacle.

To avoid the pulse shape problem it was necessary to run the laser at low power (Fig. 3.4B). With $\sim 14 \mathrm{~mW}$ through the fiber the frequency spectrum became more symmetric and a clean short pulse of 220 fs was obtained. The output power, however, was too low to get efficient second harmonic generation to do TPPE experiments.

In the end strategic decisions concerning our ultimate goal of time-resolved TPPE spectroscopy had to be made. It was concluded that pulse compression was not feasible for our particular use and the emerging solid state ultrafast lasers represented a much more attractive choice.

\subsubsection{Femtosecond Ti:Sapphire Laser System}

Since mode-locking was demonstrated in the titanium doped sapphire laser system in the late 1980 s $[76,77,78]$, solid-state media like the Ti:sapphire laser has been supplanting dye based systems as the most popular ultrafast sources. Several characteristics make Ti:sapphire a better alternative ultrafast source. The sapphire host is easy to handle, free from chemical degradation, and has a better thermal conductivity than dye solution allowing it to handle substantially higher pump powers. The lack of a flowing dye solution increases the stability of the Ti:sapphire laser. In addition, the demonstration of a $250 \mathrm{kHz}$ Ti:sapphire regenerative amplifier [79] and the combination with an optical parametric amplifier (OPA) [80] allow the production of femtosecond laser pulses at a high repetition rate with a wide tunability. 
Because of the difficulties involved in the pulse compression scheme, and all of the above benefits, we decided to utilize Ti:sapphire based laser technology as our new light source.

The apparatus used in all of the experiments described in this dissertation is shown in Fig. 3.5. A Coherent RegA 9000 Ti:sapphire regenerative amplifier, seeded by a Coherent Mira 900 Ti:sapphire oscillator, produces a $200 \mathrm{kHz}$ train of $240 \mathrm{fs}$ pulses (autocorrelation FWHM) at $800 \mathrm{~nm}$. A Coherent OPA 9400 optical parametric amplifier uses part of the $800 \mathrm{~nm}$ beam to generate white-light continuum while the frequency-double remainder is used to parametrically amplify a portion of the continuum in a BBO crystal. The positively-chirped pulses from the OPA are further compressed with SF-10 prisms in a four-pass configuration to yield $50 \mathrm{~nJ}$ pulses with 100 fs autocorrelation FWHM at $600 \mathrm{~nm}$, as shown in Fig. 3.6. The OPA output has been tuned in our laboratory over the range 470 to $730 \mathrm{~nm}$. Visible pulses are doubled with $\sim 15 \%$ conversion efficiency in a $300 \mu \mathrm{m} \mathrm{BBO}$ crystal. As shown below in Sect. 4.3, a UV pump/visible probe TPPE instrument function of 110 to $120 \mathrm{fs}$ is routinely achieved for UV wavelengths near $300 \mathrm{~nm}$. No transmissive optics are used in the UV path except the collimating lens after the SHG crystal and the chamber window. The energy resolution for spectra taken with femtosecond pulses is dominated by the bandwidth of the laser pulse, which is $31 \mathrm{meV}$ at $600 \mathrm{~nm}$. The energy widths of the TPPE peaks associated with the image state range from 40 $70 \mathrm{meV}$ FWHM for emission normal to the surface. 


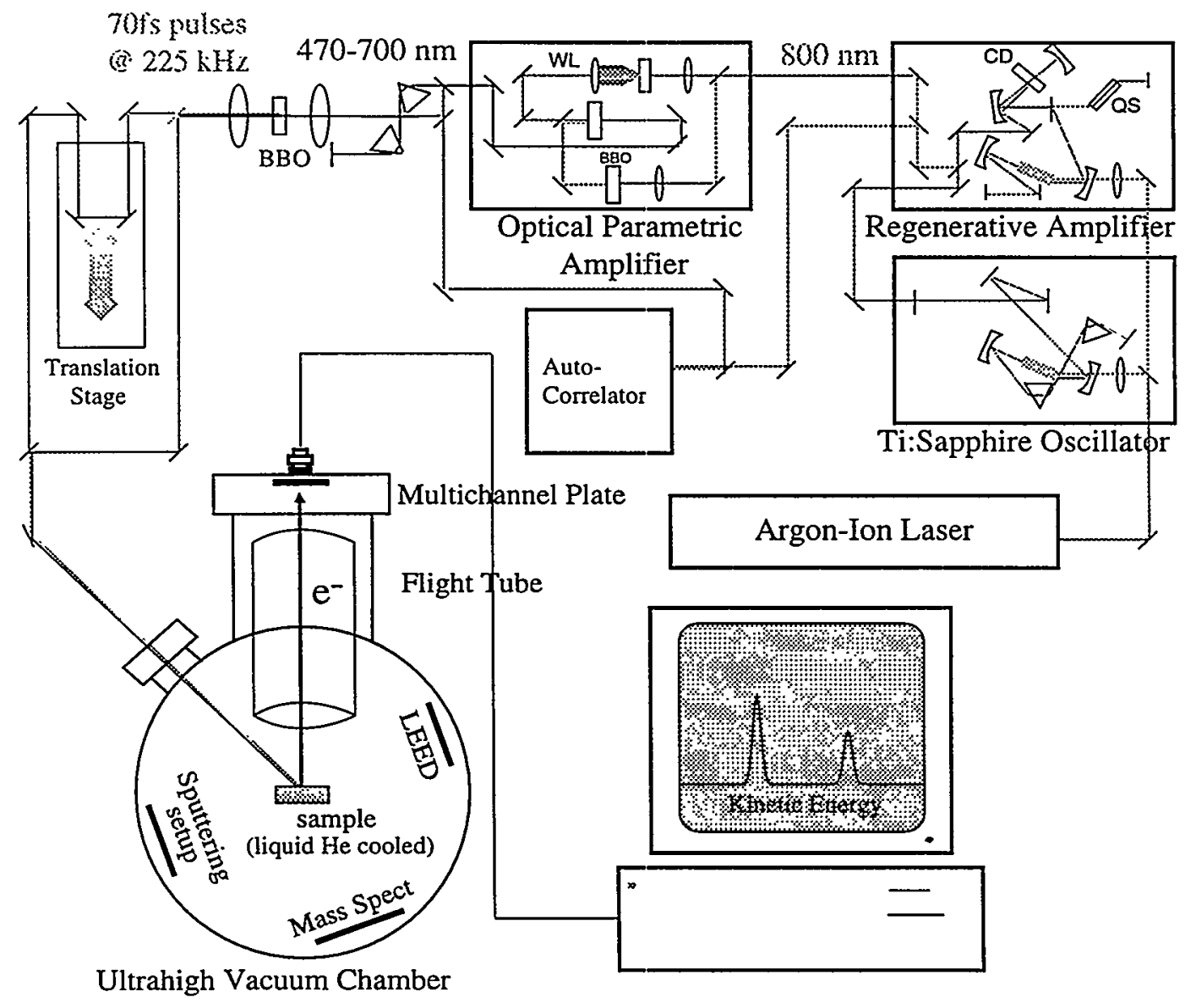

Figure 3.5: Femtosecond time- and angle-resolved TPPE spectrometer 


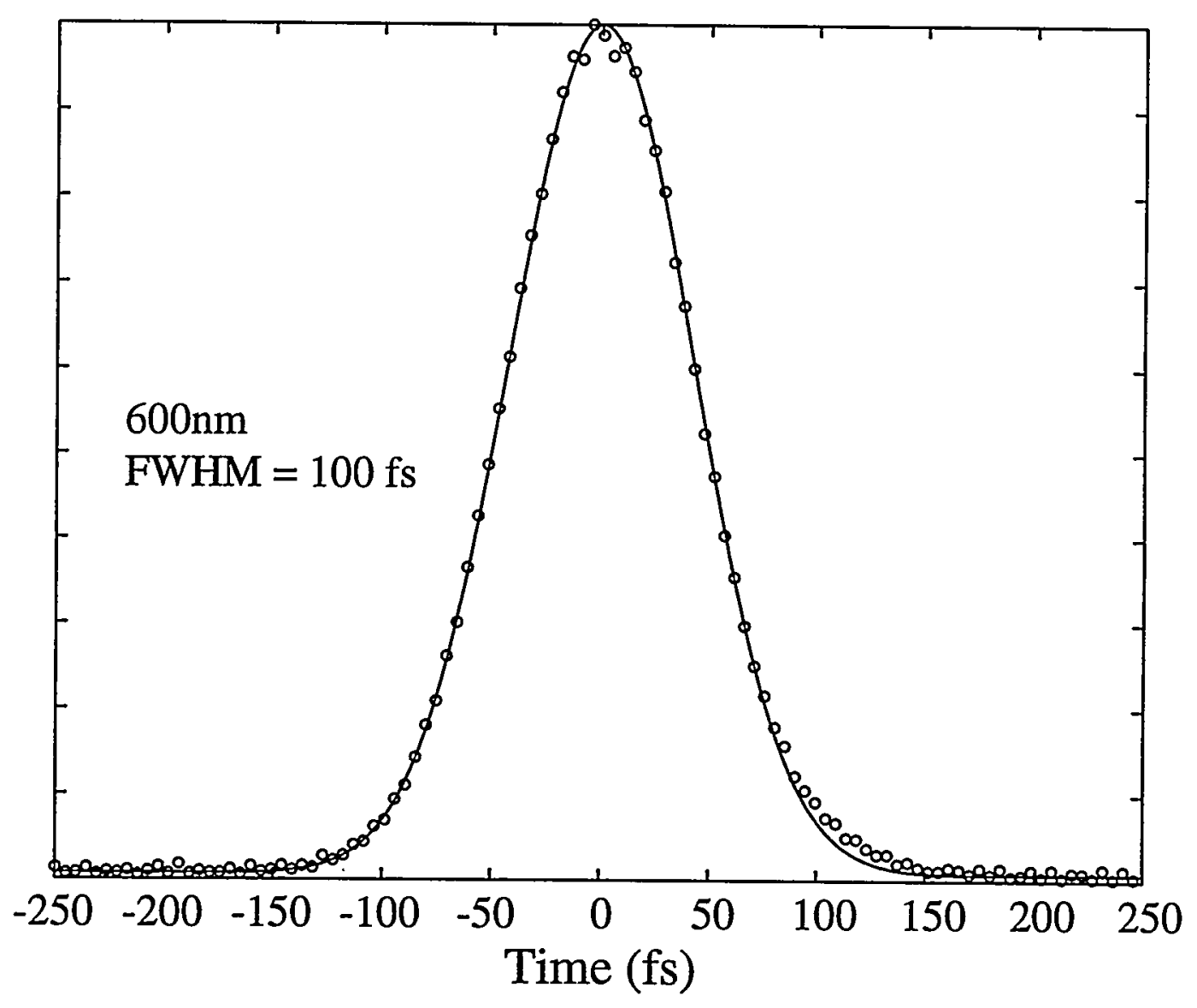

Figure 3.6: Autocorrelation of the $600 \mathrm{~nm}$ pulse from the optical parametric amplifier. The circles are the data and the solid line is a Gaussian fit. The FWHM is 100 fs. 
Chapter 3. Experimental

The time-resolved decay curve for the feature of interest is obtained by plotting the photoelectron counts per second at a given energy versus time delay between the UV and visible pulses. Because we use time-correlated single-electron counting detection, it is convenient to collect a full energy distribution curve at each time delay. In order to average out the effect of laser power drift, we take several sets of scans and add them together, running the delay stage both forward and backward.

\subsection{Sample Preparation}

The crystal of $\operatorname{Ag}(111)$ was previously obtained from Monocrystals, Inc. and polished by standard metallographic and chemical techniques. The preparation procedure has been described in details previously in Walt Merry's thesis [71]. The orientation of the sample on the goniometer sector was set to allow angle-resolved measurements along the $\bar{\Gamma}$ to $\bar{K}$ direction in the $\mathrm{Ag}(111)$ surface Brillouin zone. Immediately before each experiment it was cleaned by sputtering with $500 \mathrm{~V}$ argon ions at $500 \mathrm{~K}$ for 20 mins and followed by annealing at $725 \mathrm{~K}$. Absence of contamination was verified by Auger spectrometry and sharp, well-ordered LEED patterns were obtained. The ultrahigh vacuum chamber background pressure was $\sim 2 \times 10^{-10}$ Torr.

The sample was cooled by means of a liquid helium cryostat resulting in a minimum sample temperature of $40-50 \mathrm{~K}$. Alkane layers were grown by exposing the cold $\mathrm{Ag}(111)$ crystal surface to the vapor. Liquid n-alkane was obtained from EM Industries, Inc., spectrophotometry grade. Prior to leak the vapor into the chamber 
the liquid was degassed by three freeze-thaw cycles in a sample line which has a base pressure of $1 \times 10^{-7}$ Torr. Layer-by-layer growth is monitored in situ in the TPPE spectrum [70]. During the growth of a monolayer, both the clean surface $n=1$ image state peak and the clean surface state peak gradually disappear, while a monolayer $n=1$ image state peak grows in at a different energy. Further dosing yields bilayer coverage. Dosing typically results in uneven surface coverage, with patches of bilayer, monolayer, and bare $\mathrm{Ag}(111)$ coexisting on the surface, each clearly distinguished in the TPPE spectrum [81]. For n-heptane/Ag(111), bilayer can be driven off by annealing at $143 \mathrm{~K}$, leaving pure monolayer coverage. Trilayer can be desorbed at $135 \mathrm{~K}$, leaving uniform bilayer coverage. All experiments on $n$-heptane layers reported in the later chapters were conducted after dosing, annealing at the appropriate temperature, and subsequent cooling to the experimental temperature range. A similar procedure was followed for n-octane. n-Alkanes grow ordered layers on the $\mathrm{Ag}(111)$ surface with the carbon-carbon bonds parallel to the surface (an all-trans conformation) as determined by LEED studies [82]. 


\section{Chapter 4}

\section{Electron Dynamics on a Bare}

\section{$\operatorname{Ag}(111)$ Surface}

Examination of the properties of the image states on bare $\operatorname{Ag}(111)$ with the newly implemented femtosecond light source was necessary to interpret results obtained from femtosecond TPPE experiments on this surface when covered by adsorbate layers. A wavelength survey was performed to map out the various two-photon excitations which can be observed. Attention was then focussed on the spectral features assigned as the image states. The dispersion relation of the $n=1$ image state were measured. The lifetime of the $n=1$ and 2 image states were measured and compared to those of other workers. The temperature and angle dependence of the lifetime were investigated. These results revealed the performance of our femtosecond TPPE spectrometer and established a scientific base to judge the significance of novel femtosecond results 
on metal-dielectric systems.

\subsection{Wavelength Survey}

Figure 4.1 shows a set of photoelectron kinetic energy spectra collected at a series of laser wavelengths and at room temperature. Photoelectrons emitted normal to the surface were detected. The delay time between the visible and UV pulses were set to zero. Excitation scheme are shown in Fig. 4.2.

The most prominent series of peaks labeled $I_{1}$ are assigned to the $n=1$ image state. This state is excited from continuum bulk levels below the Fermi energy by absorbing a UV photon. Because the $n=1$ image state is an intermediate state of fixed energy, as the photon energy is scanned, the photoelectron kinetic energy will change to the same extent as the energy of the photoemitting photon. A plot of the kinetic energy versus visible photon energy (Fig. 4.2) shows a slope of 1 , indicating that it is photoemitted by a visible photon.

In collecting these data, a DC bias was applied to flight tube to offset the difference in work function between the sample and detector. This bias was selected empirically to make the $n=1$ peak match the binding energy of $-0.77 \mathrm{eV}$ reported by Giesen et al. [50] for the $\operatorname{Ag}(111)$ surface.

The spectral feature labelled $S$ has a different excitation mechanism from the $n=1$ image state. The peak energy varies more rapidly with changes in photon energy. This state is assigned to the occupied L-gap surface state. Because the 


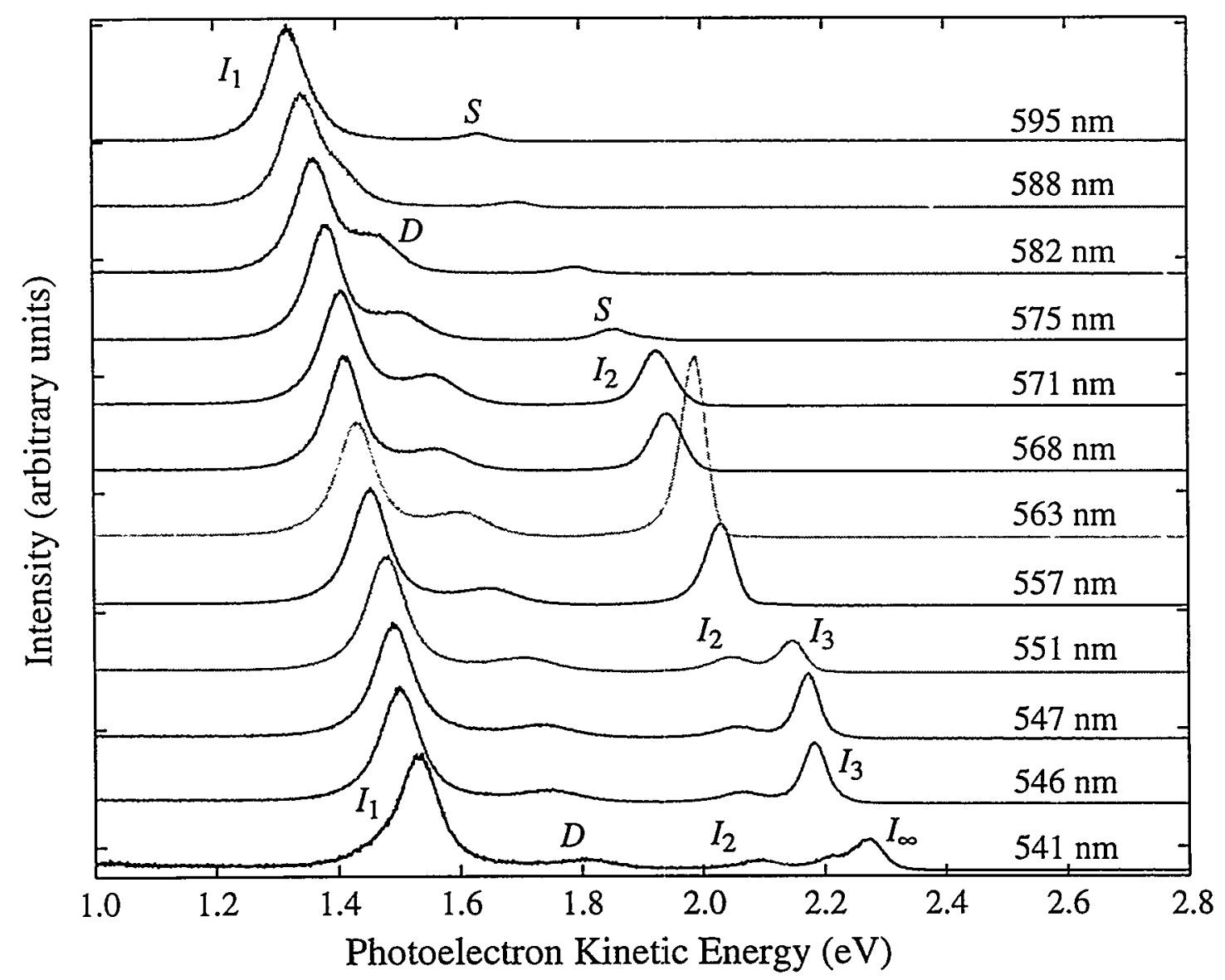

Figure 4.1: TPPE kinetic energy spectra of $\mathrm{Ag}(111)$ at a series of wavelengths. The kinetic energy range of $1-2.7 \mathrm{eV}$ is shown. The delay time between the visible and UV pulses were set to zero. 
(c)

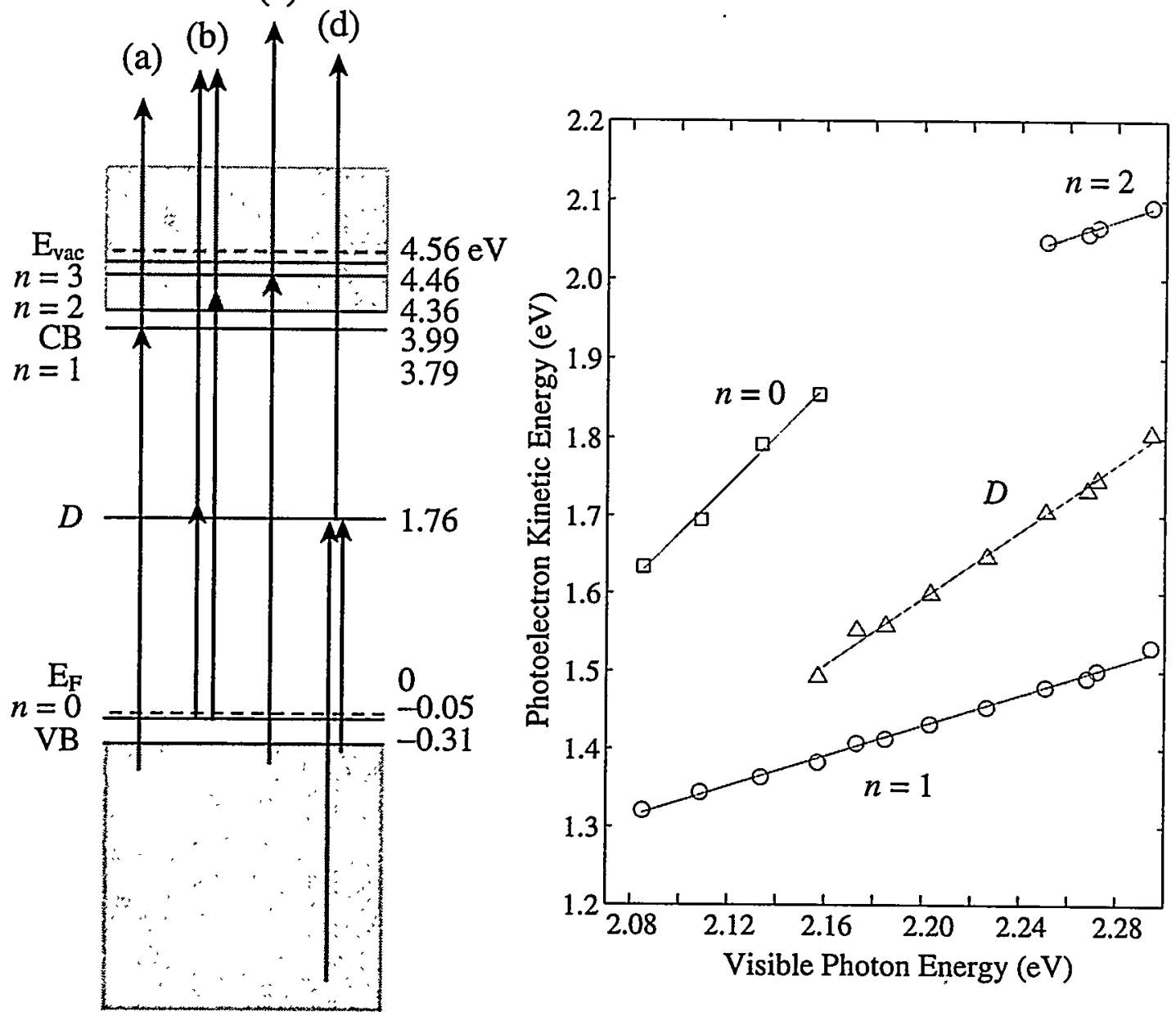

Figure 4.2: Excitation scheme of bichromatic TPPE at a $\mathrm{Ag}(111)$ surface. In (a), the $n=1$ image state is excited from occupied bulk bands by a UV photon, and ejected by a visible photon. In (b), the photoemission from the occupied surface state occurs through a non-resonant two-photon process. In (c), as the photon energy increases, the $n=2$ state can be resonantly excited from the surface state and then from bulk bands. In (d), the feature $D$ is excited from occupied bulk bands. This feature is attributed to the surface state on $\mathrm{Ag}(110)$ facets. These states show a different dependence of the kinetic energy with visible photon energy. 
photoelectron peak is due to nonresonant two-photon photoemission (one UV and one visible) from an initial state of constant energy, the shift in peak energy upon variation of the photon energy exhibits a slope of 3 (Fig. 4.2). Subtracting three times the visible photon energy from the kinetic energy, and taking the work function to be $4.56 \mathrm{eV}$ [83], the energy position of the surface state is determined to be $-0.06 \mathrm{eV}$ below $E_{F}$, in agreement with the literature value of -0.05 obtained by TPPE [83] and -0.12 by high resolution UV photoemission [84].

Excitation with higher photon energies produced a series of peaks labelled as $I_{2}$ and were assigned to the $n=2$ image state. Its intensity is most prominent near wavelength of $563 \mathrm{~nm}$ due to resonant excitation from the surface state, and stays relatively constant when moves out of resonance. Its mode of excitation is the same as the $n=1$ state, showing a slope of 1 in Fig. 4.2. From the difference between the kinetic energy and photon energy, its binding energy relative to the vacuum level is found to be $-0.21 \mathrm{eV}$, in good agreement with the previously measured value of $-0.23 \mathrm{eV}[8]$. As evident at the bottom of Fig. 4.1, the $n=3$ and higher quantum states sequentially moves into resonance with the surface state and exhibit pronounce intensity enhancement, as the photon energy increases.

An alternate method of determining the binding energies of the image states is to analyze the convergence of the image series to the vacuum level using the quantum defect formula. The energy separation between two or more image states can be used to determine the quantum defect $a$ in Eqn. 2.7 on page 10. The binding energies for 
the $n=1$ and 2 image states are determined to be -0.77 and $-0.20 \mathrm{eV}$, respectively, and $a=0.051$. This method can also be used to determine the local work function [52] and the work function shift $[85,86]$ upon adsorption of overlayers.

The feature labelled $D$ exhibits a slope of 2 in Fig. 4.2. The energy of this state is $1.76 \mathrm{eV}$ above $E_{F}$ determined by subtraction of two times the visible photon energy from the kinetic energy. This state is currently of unknown origin but tentatively assigned to the surface state on $\mathrm{Ag}(110)$ facets. A similar state of $1.92 \mathrm{eV}$ above $E_{F}$ were also observed on $\operatorname{Ag}(111)$ by Steinmann and coworkers [7]. The $\vec{Y}$ gap surface state on $\mathrm{Ag}(110)$ is located between 1.6 and $2.5 \mathrm{eV}$ above $E_{F}$ as determined by inverse photoemission $[87,88]$ and electroreflectance [89].

Figure 4.3 shows a subset of the wavelength survey data for the higher kinetic energy region. Similar behavior is observed except that now the slope of the kinetic energy versus visible photon energy increases by 1 for all states. For the image states and surface state, this corresponds to a TPPE excitation process with two UV photons.

The small broad feature labelled $A$ does not shift with photon energy. This feature is attributed to an energy pooling process [7] in which two electrons in the image state interact just like in an Auger process: one electron returning to the ground state state gives its energy to the second electron, which is emitted, giving rise to the peak $A$. The energy of the emitted electron should be independent of photon energy, and equal to the work function subtracting twice the image state binding energy, $3.02 \mathrm{eV}$. The 


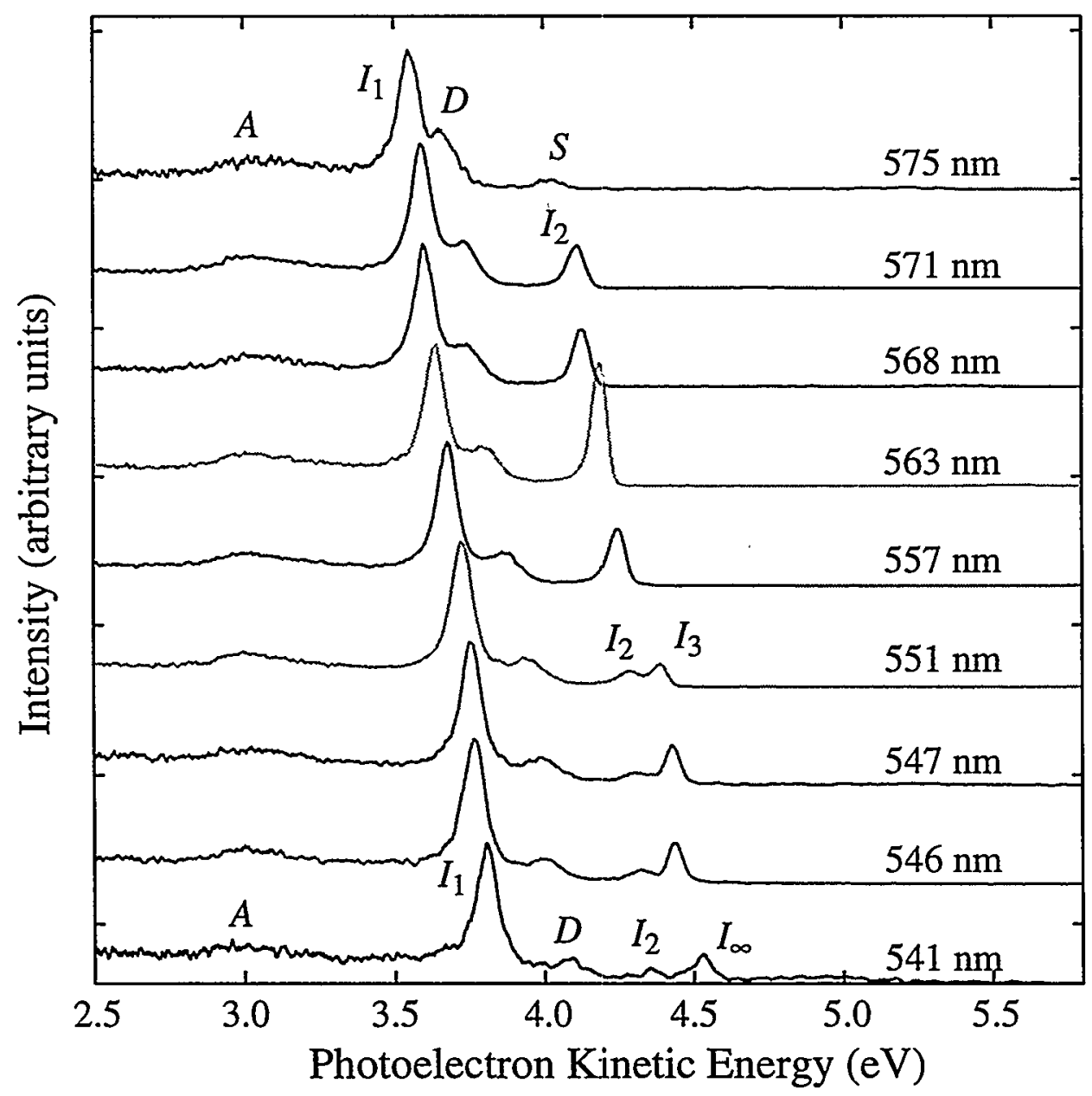

Figure 4.3: TPPE kinetic energy spectra of $\mathrm{Ag}(111)$ at a series of wavelengths. The kinetic energy range of $2.5-5.8 \mathrm{eV}$ is shown. The delay time between the visible and UV pulses were set to zero. 
position of peak $A$ is consistent with this picture.

\subsection{Dispersion Relation}

The dispersion of the $n=1$ image state was determined by angle-resolved TPPE. The experiments were conducted at UV/visible wavelengths of $290 \mathrm{~nm} / 580 \mathrm{~nm}$ such that the excitation of the $n=1$ image state originates from the continuum of occupied bulk states below the projected bulk band gap. Figure 4.4 shows the photoelectron kinetic energy distributions taken at a series of sample rotation angles and at room temperature. The delay time between the visible and UV pulses were set to zero. Dispersion of the $n=1$ image state, feature $D$ and the surface state is evident. The surface state quickly loses intensity as the rotation angle increases. This effect is expected because the occupied surface state has an effective mass of $0.53 m_{\mathbf{e}}$ [84]. Thus it crosses $E_{F}$ and becomes unoccupied at $k_{\|}=0.08 \AA^{-1}$. Another recent study determined the effective mass to be $0.44 \pm 0.04 m_{\mathrm{e}}[90]$.

Using Equations 3.1, the kinetic energy of the $n=1$ image state is plotted as a function of parallel momentum in Fig. 4.5A. A fit to the parabolic dispersion relation, Eqn. 3.2, gives an effective mass of $1.3 \pm 0.1 m_{\mathrm{e}}$. This agrees well with the value of $1.3 \pm 0.15 m_{\mathrm{e}}$ reported in the literature [50]. As discussed previously in Sect. 2.3, the effective mass is somewhat heavier than the mass of a free electron as a result of the influence of the relatively flat $\mathrm{Ag}(111)$ surface-projected conduction band.

The effective mass of the feature $D$ is determined to be $0.56 \pm 0.15 m_{\mathrm{e}}$ (Fig. 4.5B), 


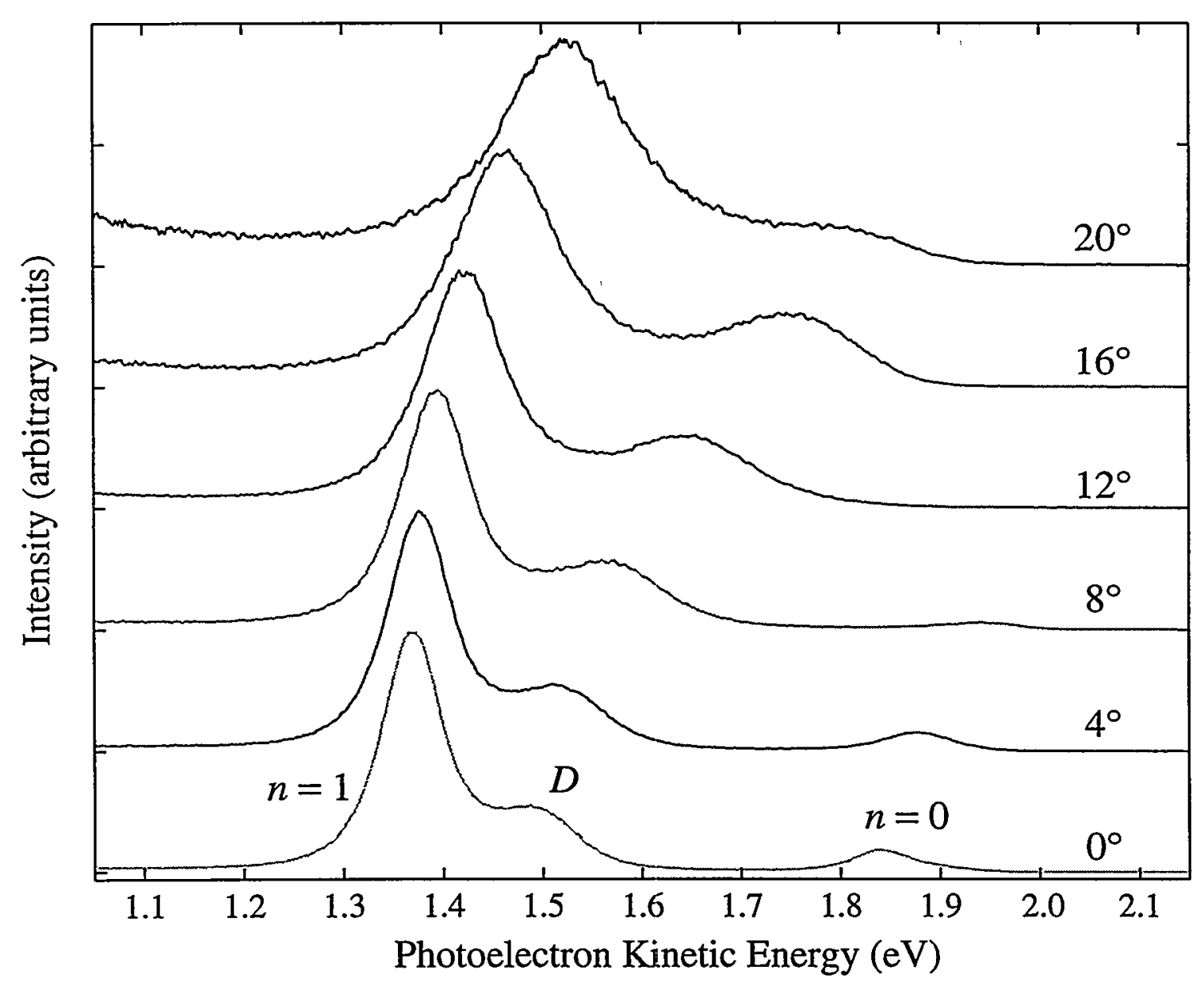

Figure 4.4: TPPE spectra collected at a series of angles between the sample normal and the detector axis. The experiments were conducted at room temperature with UV/visible wavelengths of $290 \mathrm{~nm} / 580 \mathrm{~nm}$. The delay time between the visible and UV pulses were set to zero. 


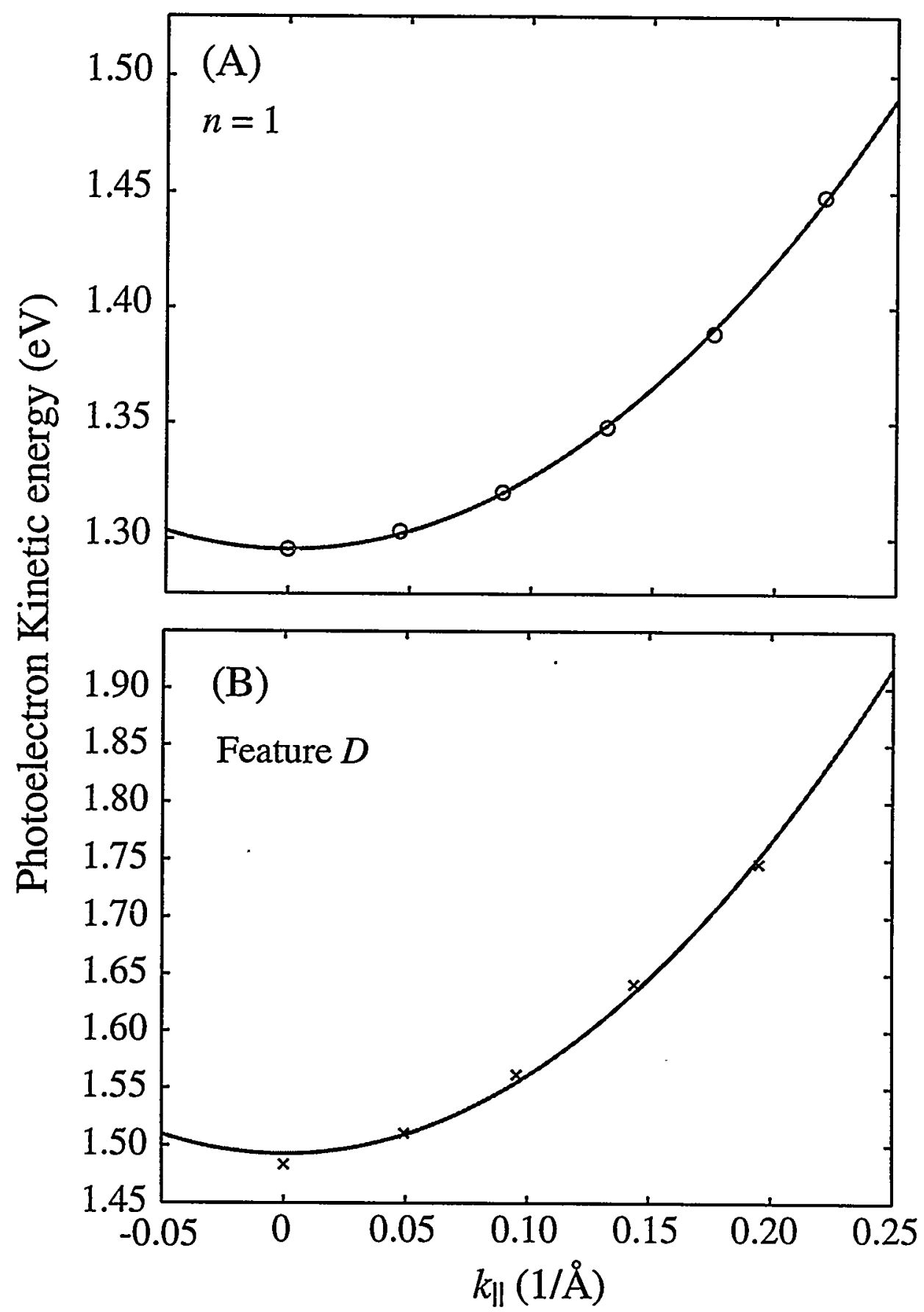

Figure 4.5: (A) Reduced dispersion data for the $n=1$ state are shown along with a parabolic fit determining the experimental effective mass, yielding $m^{*}=1.3 \pm 0.1 m_{\mathrm{e}}$. (B) The dispersion relation for feature $D$ with $m^{*}=0.56 \pm 0.15 m_{\mathrm{e}}$. 
somewhat smaller than the $0.9 \pm 0.2 m_{\mathrm{e}}$ determined for the surface state on $\mathrm{Ag}(110)$ [87].

\section{$4.3 \quad$ Lifetime Measurements}

The lifetime of the image states were determined by time-resolved TPPE. Figure 4.6 is a set of the photoelectron kinetic energy distributions taken at a series of time delays between UV and visible pulses. The time delay is defined as positive when the visible pulses arrives after the UV. The experiments were conducted at $\mathrm{UV} /$ visible wavelengths of $300 \mathrm{~nm} / 600 \mathrm{~nm}$. The sample temperature was $106 \mathrm{~K}$ and the sample rotation angle was zero. The $n=1$ image state and the surface state peaks are well separated. Their time-resolved dynamic traces shown in Fig. 4.7 were obtained by plotting the photoelectron counts per second at the corresponding peak maxima versus time delay.

We obtain an instrument function for the time-resolution of our apparatus by taking advantage of the surface state peak that is due to simultaneous two-photon absorption. The intermediate state for this process is a virtual state whose lifetime can be taken as zero. The time dependence of this peak therefore serve as a crosscorrelation of the UV pump and visible probe laser pulses at the sample, including all dispersion introduced by the optics and the UHV chamber window. The instrument function obtained in this way is well-fit by a Gaussian, typically has FWHM of 110 to $120 \mathrm{fs}$ for wavelengths near $600 \mathrm{~nm}$ as shown in Fig. 4.7, and provides a determination of $t=0$ (time delay at which the UV and the visible pulses are directly overlapped). 


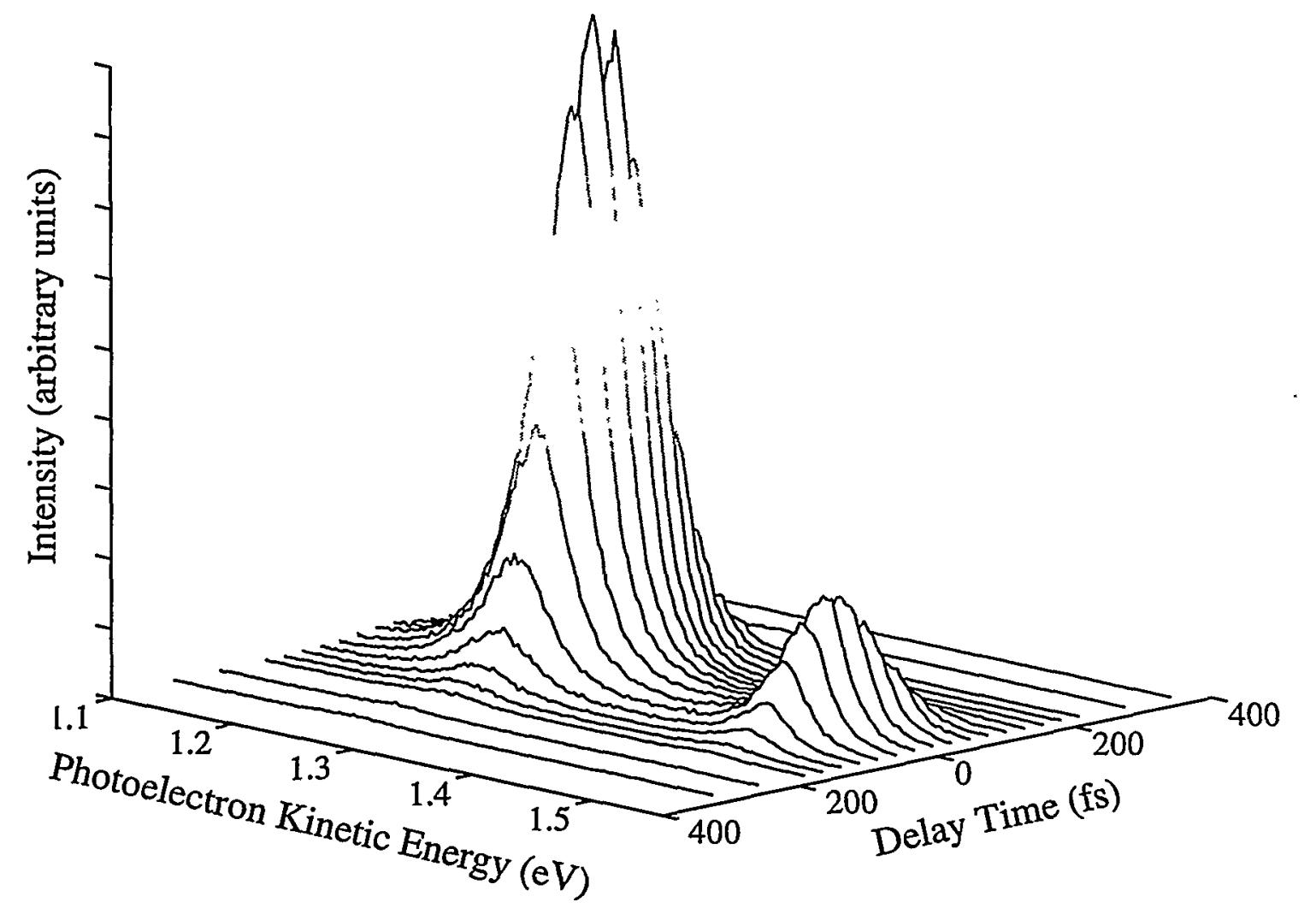

Figure 4.6: The photoelectron energy distribution curves for bare $\mathrm{Ag}(111)$ excited with $300 \mathrm{~nm}$ and photoemitting with $600 \mathrm{~nm}$ at various pump-probe delay and at a sample temperature of $106 \mathrm{~K}$. The small peak is due to simultaneous TPPE from the occupied surface state. The large peak is due to electrons which transiently populate the $n=1$ image potential state. 


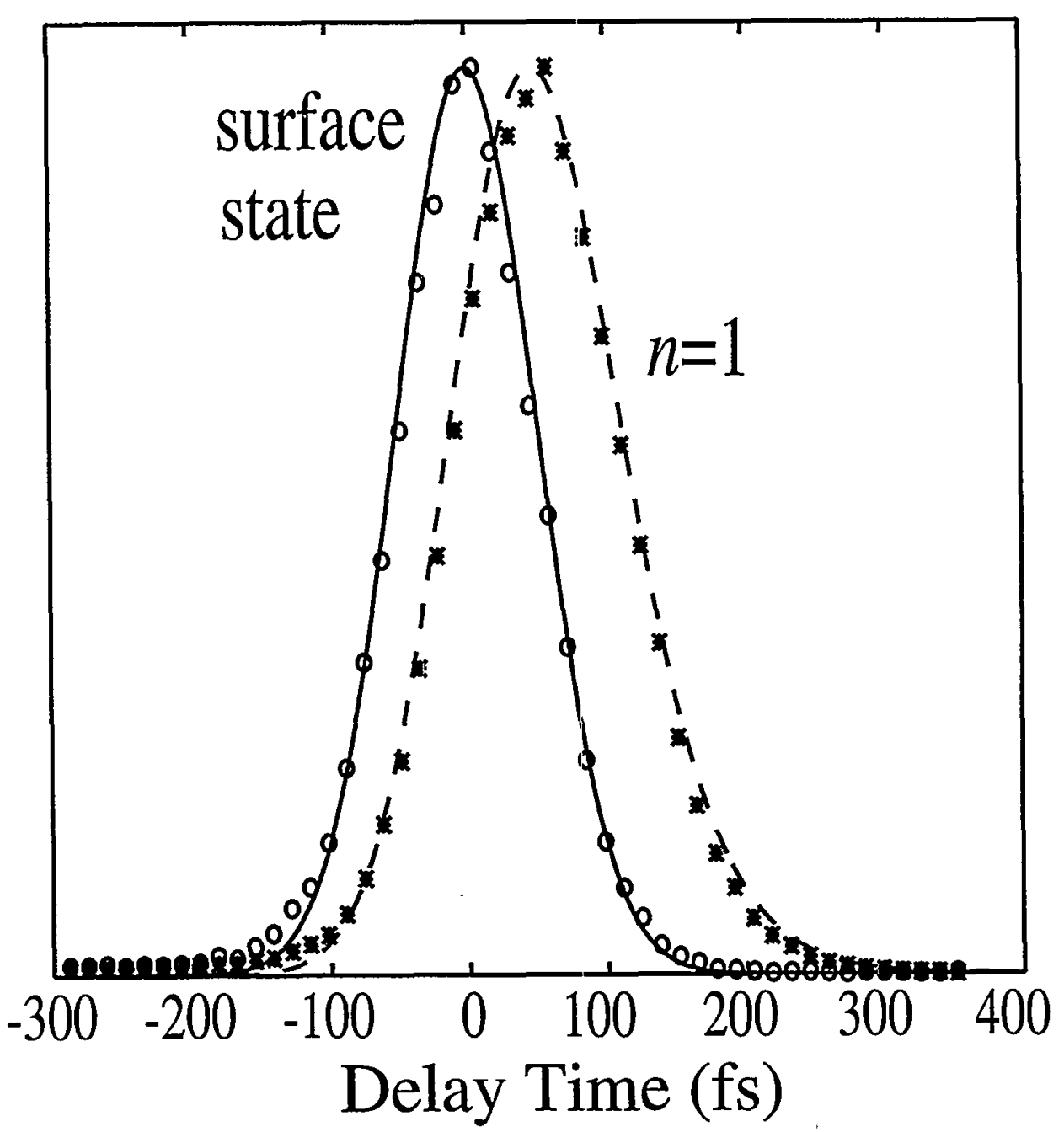

Figure 4.7: The dynamics traces of the $n=1$ and surface states. The Gaussian FWHM of the surface state signal is $120 \mathrm{fs}$ and is assumed to be the instrument function. The rise and decay times for the $n=1$ signal are deconvolved using the Gaussian fit to the instrument function and the kinetic model represented by Equation 4.1 on the next page to yield a rise time $\tau_{1}=25$ fs and a lifetime $\tau_{2}=32 \pm 5$ fs. 
The instrument function thus obtained is convolved with a kinetic model of the interface electronic state dynamics to fit the data. In order to obtain good fits to the image state dynamic traces, it is necessary to use a model incorporating both a rise time $\left(\tau_{1}\right)$ and a decay time $\left(\tau_{2}\right)$. We have assumed a single level of interest, initially unpopulated, which is fed at rate $k_{1}=\tau_{1}^{-1}$ and decays at rate $k_{2}=\tau_{2}^{-1}$. The functional form for the solution to these rate equations is

$$
N\left(\tau_{1}, \tau_{2} ; t\right) \propto \frac{k_{1}}{k_{2}-k_{1}}\left[\exp \left(-k_{1}\left(t-t_{0}\right)\right)-\exp \left(-k_{2}\left(t-t_{0}\right)\right)\right]
$$

where $N\left(\tau_{1}, \tau_{2} ; t\right)$ is the population in the level of interest, and $t_{0}$ is unambiguously determined from the dynamics of the non-resonant surface state peak. The best fit of the $n=1$ image state yields a rise time of $24 \mathrm{fs}$ and a decay time of $32 \pm 5 \mathrm{fs}$. The rise time may be related to $k_{\|}$relaxation to lower $k_{\|}$states within the image state band. A portion of the rise time may be due to the coherence dephasing $[68,91,43]$. It is not as yet understood.

Lifetime measurements have been conducted at room temperature with UV/visible wavelengths of $273 \mathrm{~nm} / 545 \mathrm{~nm}$ in order to investigate both $n=1$ and $n=2$ states. At this wavelength, the surface state peak is not available in the spectrum because it is on resonance near resonance with the $n=3$ state. It was found from timeresolved experiments conducted at wavelength around $580 \mathrm{~nm}$ that the feature $D$ is very short lived and has a dynamic trace very much the same as the surface state. 


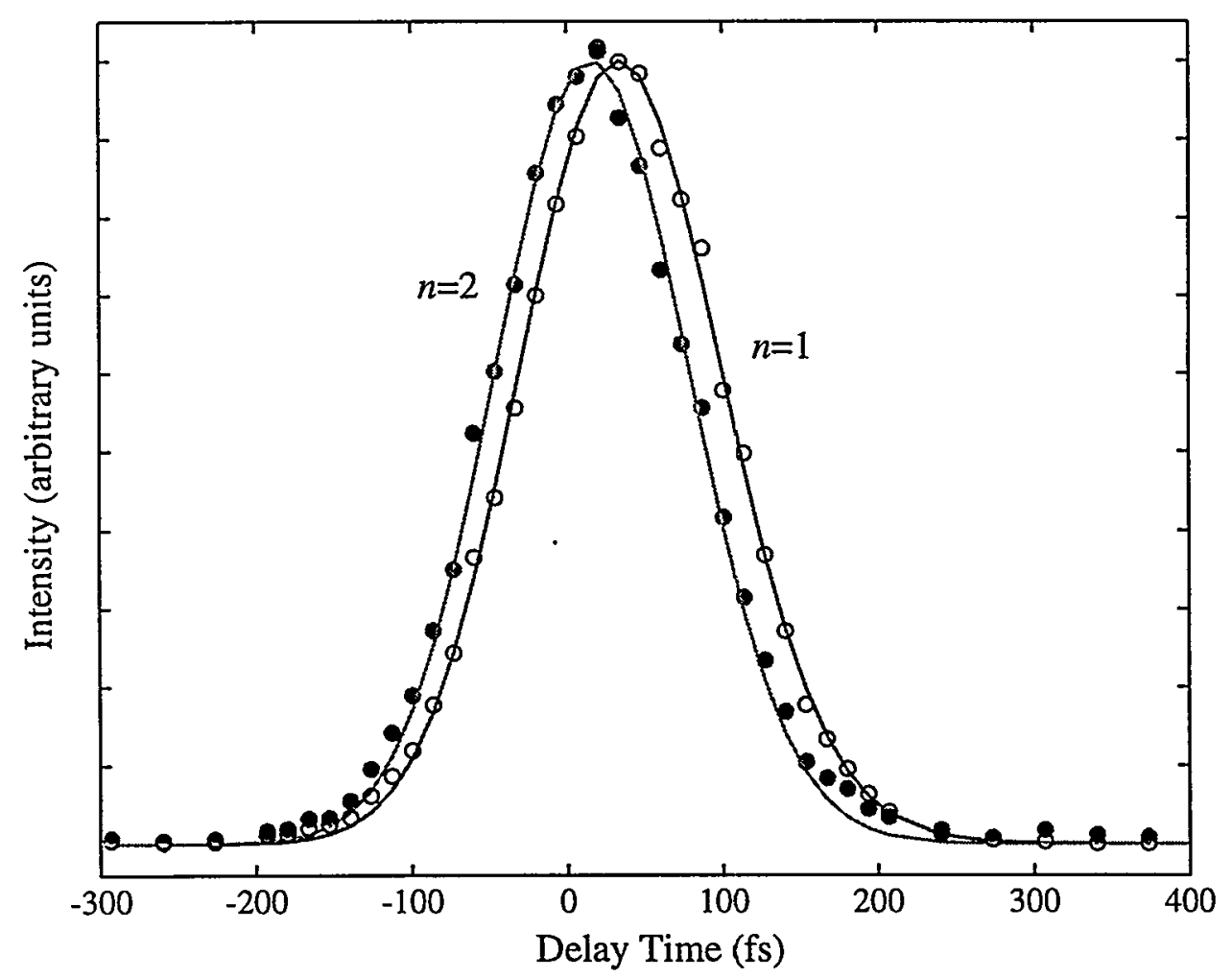

Figure 4.8: The dynamics traces of the $n=1(\circ)$ and $n=2(\bullet)$ image states on $\mathrm{Ag}(111)$. The rise and decay times are $15 \mathrm{fs}$ and 22 fs for the $n=1$ state, respectively, and $1 \mathrm{fs}$ and 16 fs for the $n=2$ state, respectively. 
The short lifetime is expected because it is $1.8 \mathrm{eV}$ above $E_{F}$. Therefore, the time dependence of the feature $D$ can also serve as the instrument function in this case. The dynamic traces for $n=1$ and 2 and the fits are shown in Fig. 4.8. In agreement with Schoenlein et al. [39], we find that the lifetime is longer for $n=1$ than for $n=2$. This indicates that the lifetimes on $\operatorname{Ag}(111)$ are dominated by the position of the image states relative to the projected bulk band structure. The $n=1$ state is located in the relative gap, while the $n=2$ and higher members of the Rydberg series are degenerate with the bulk conduction band. The lifetime trend is thus different from the approximate $n^{3}$ dependence observed for $\operatorname{Ag}(100)$ [38], where both $n=1$ and 2 states are within the gap and the lifetimes were measured by Schoenlein et al. [39] to be 25 fs and 180 fs for $n=1$ and 2 , respectively.

\subsection{Temperature and Angle Dependence of Image}

\section{State Dynamics}

Figure 4.9 displays TPPE photoelectron kinetic energy spectra taken at various temperatures. The wavelengths of UV/visible pulses were $308 \mathrm{~nm} / 615 \mathrm{~nm}$ and the time delay between them were set to zero. Photoelectrons emitted normal to the surface were detected. The $n=1$ image state peak appears at lower kinetic energies than the surface state. With decreasing temperature the peak maxima of both the 


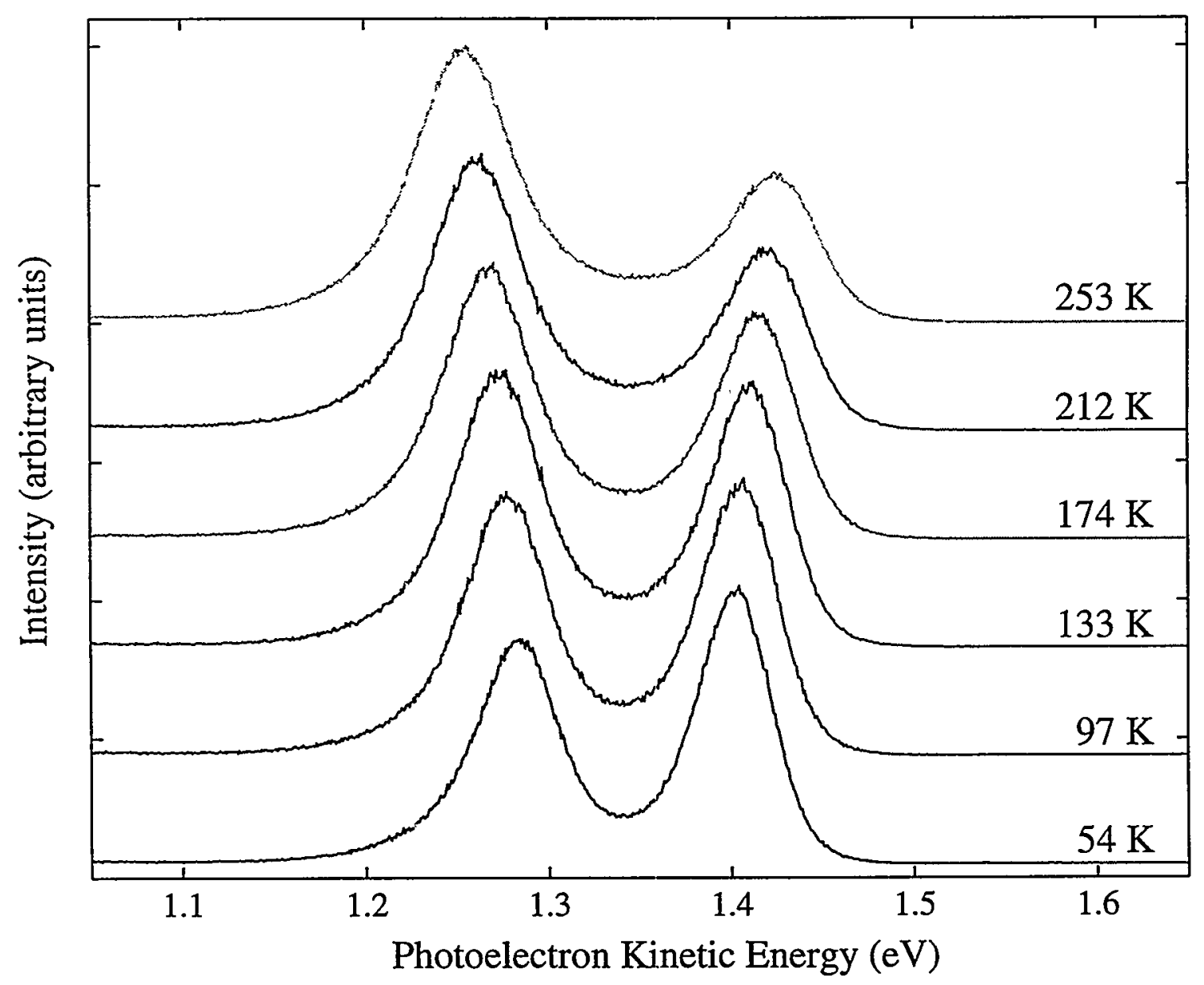

Figure 4.9: TPPE spectra from $\mathrm{Ag}(111)$ for various temperatures taken at UV/visible wavelengths of $308 \mathrm{~nm} / 615 \mathrm{~nm}$. The delay time between the visible and UV pulses were set to zero. 


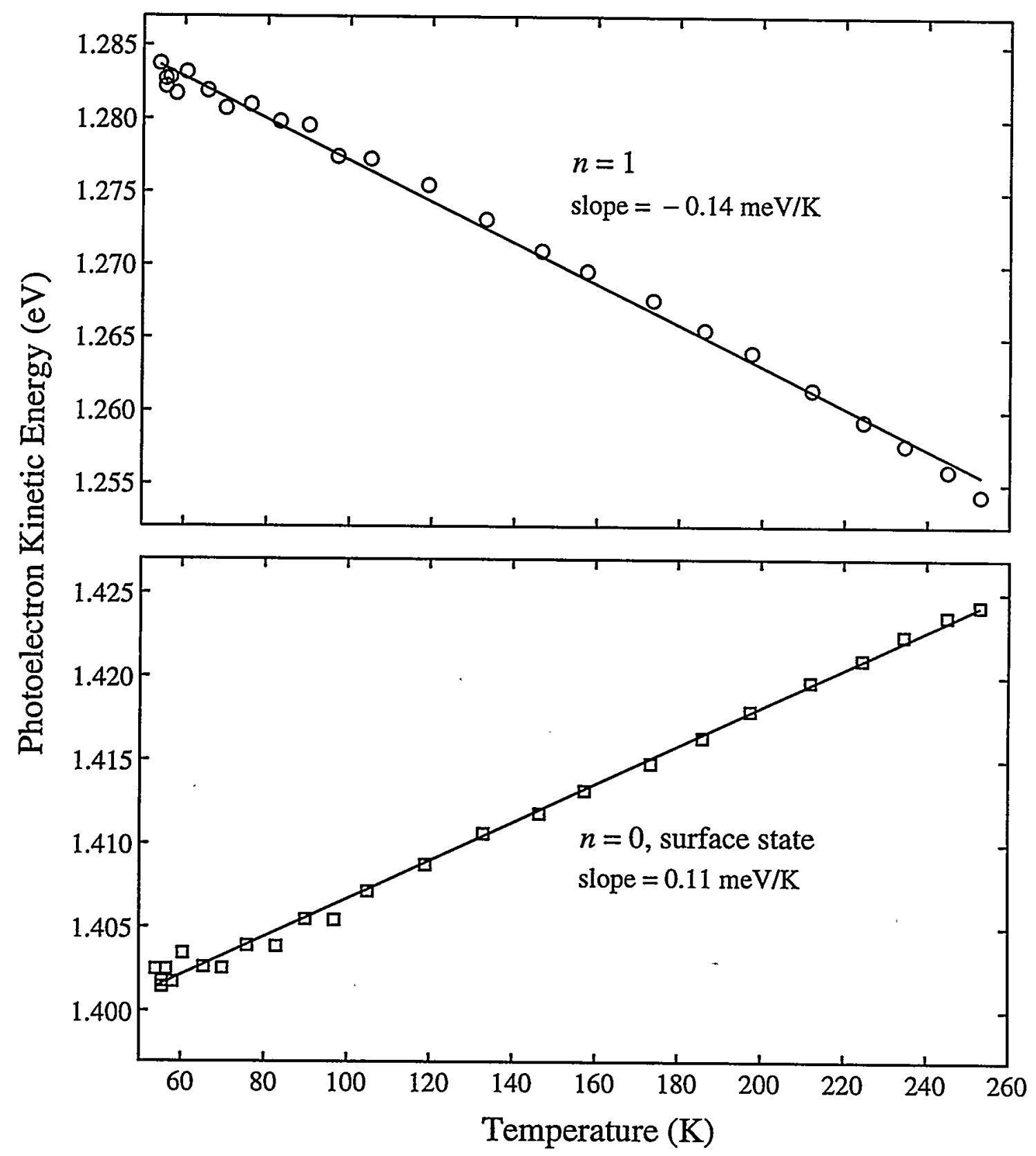

Figure 4.10: Photoelectron kinetic energy of the $n=1$ image state (top) and the surface state (bottom) on $\mathrm{Ag}(111)$ as a function of temperature. 
Similar shifts in the peak position of the $n=1$ image state have been observed on $\mathrm{Cu}(100)$ [92] and $\mathrm{Cu}(111)$ [93] by two different research groups and attributed to two different reasons. In Ref. [92] the authors concluded that the shifts are mainly due to the thermally induced work function change [94] and image state is pinned to the vacuum level with a binding energy insensitive to temperature. In Ref. [93] the authors assumed that the work function is practically independent of temperature, and the shifts are attributed to the thermally induced lattice compression that leads to shifting of the projected bulk band edges. In fact, Goldmann and coworkers [90] has successfully reproduced the temperature dependence of the occupied surface state on the (111) surfaces of noble metals based on the above assumptions.

Clearly, experimental or theoretical information on the temperature dependence of the work function and the bulk band edges for $\mathrm{Ag}(111)$ is needed to clarify the relative importance of the two factors in determining the energy shifts. A recent Monte Carlo calculation [95] predicts a work function temperature coefficient of $-0.141 \mathrm{meV} / \mathrm{K}$ for $\mathrm{Ag}(111)$. However, quantative information on the band edges is not available for $\mathrm{Ag}$ except that the upper and lower band edge are expected to simultaneously shift away from $E_{F}$ with decreasing temperature because such behavior has been observed for $\mathrm{Cu}$ and $\mathrm{Au}[96,97,98,99]$.

To investigate the temperature and angle dependence of the $n=1$ state lifetime that will serve as a reference for the studies on dielectric overlayers, time-resolved TPPE were performed at UV/visible wavelengths of $300 \mathrm{~nm} / 600 \mathrm{~nm}$. The dynamic 
traces taken at three different temperatures for photoelectrons emitted normal to the surface are shown in Fig. 4.11A. The lifetime increases from 22 fs at $303 \mathrm{~K}$ to $33 \mathrm{fs}$ at $50 \mathrm{~K}$. If the work function can be treated as a constant, the observed lifetime lengthening can be explained by the thermally induced widening of the bulk band gap leading to a change of the penetration of the wavefunction into the metal. From the MRT formalism in Sect. 2.2, the magnitude of the imaginary part of the wavevector, $q$ (Eqn. 2.12 on page 13), is a measure of the damping of the wavefunction inside the metal. Stronger damping indicates less wavefunction penetration into the metal. An alternative way to calculate $q$ is to employ a 1-D scattering model [100]. Following the method of Ref. [100] to solve the energy of the image state, and thus $q$, as a function of the width of the band gap, it is found that the penetration of the image state into the metal decreases when the band gap widens. Because the lifetime of the image state is strongly dependent on the wavefunction penetration into the bulk [41], a corresponding lengthening of the image state lifetime is expected. Similar arguments have been used to explain the temperature dependent lifetime for $n=1$ state on $\mathrm{Cu}(111)$ [93].

On the other hand, if the band edges are temperature independent but the work function increases with decreasing temperature, the calculation of $q$ predicts a lifetime shortening because the $n=1$ becomes closer to the upper band edge resulting in increasing penetration. This suggests that the effect of work function shift on lifetime is opposite to that of band edge shift. 
An other possible factor involved in the lifetime temperature dependence is the shifting of the surface state. Reference [90] shows that on $\operatorname{Ag}(111)$ the surface state becomes more bound as the temperature is lowered, becoming more occupied. This may lead to a decrease in the relaxation channels for the image state and hence a longer lifetime.

The temperature dependence of lifetime has also been measured for image state electrons with nonzero $k_{\|}$by collecting the photoelectrons at various sample rotation angles. Figure 4.11B shows one set of the dynamic traces of the $n=1$ image state taken at $14.4^{\circ}$. The lengthening of lifetime upon lowering temperature is observed for all angles studied.

Figure 4.11C displays a subset of the angle dependence data of the $n=1$ image state taken at $51 \mathrm{~K}$. The lifetime decreases from $33 \pm 5$ fs at $0^{\circ}\left(k_{\|}=0\right)$ to $16 \pm 7 \mathrm{fs}$ at $20.4^{\circ}\left(k_{\|}=0.22 \AA^{-1}\right)$. This shortening of lifetime with increasing $k_{\|}$is also observed for data taken at $123 \mathrm{~K}$ and $303 \mathrm{~K}$, as is evident in comparing Fig. 4.11A and Fig. 4.11B. This may be due to relaxation to image states with smaller $k_{\|}[33,41]$ in which an extra linewidth broadening with a $k_{\|}^{4}$-dependence is predicted. In addition, there may be an enhancement of the rate for decaying back into the empty bulk states. On $\operatorname{Ag}(111)$, the bottom of $n=1$ image state band is only $200 \mathrm{meV}$ below the upper projected bulk band edge. With increasing $k_{\|}$, the $n=1$ state will become closer to the conduction band and eventually become resonant with the bulk continuum. Both of these processes may contribute to the observed effect. The time-resolved 


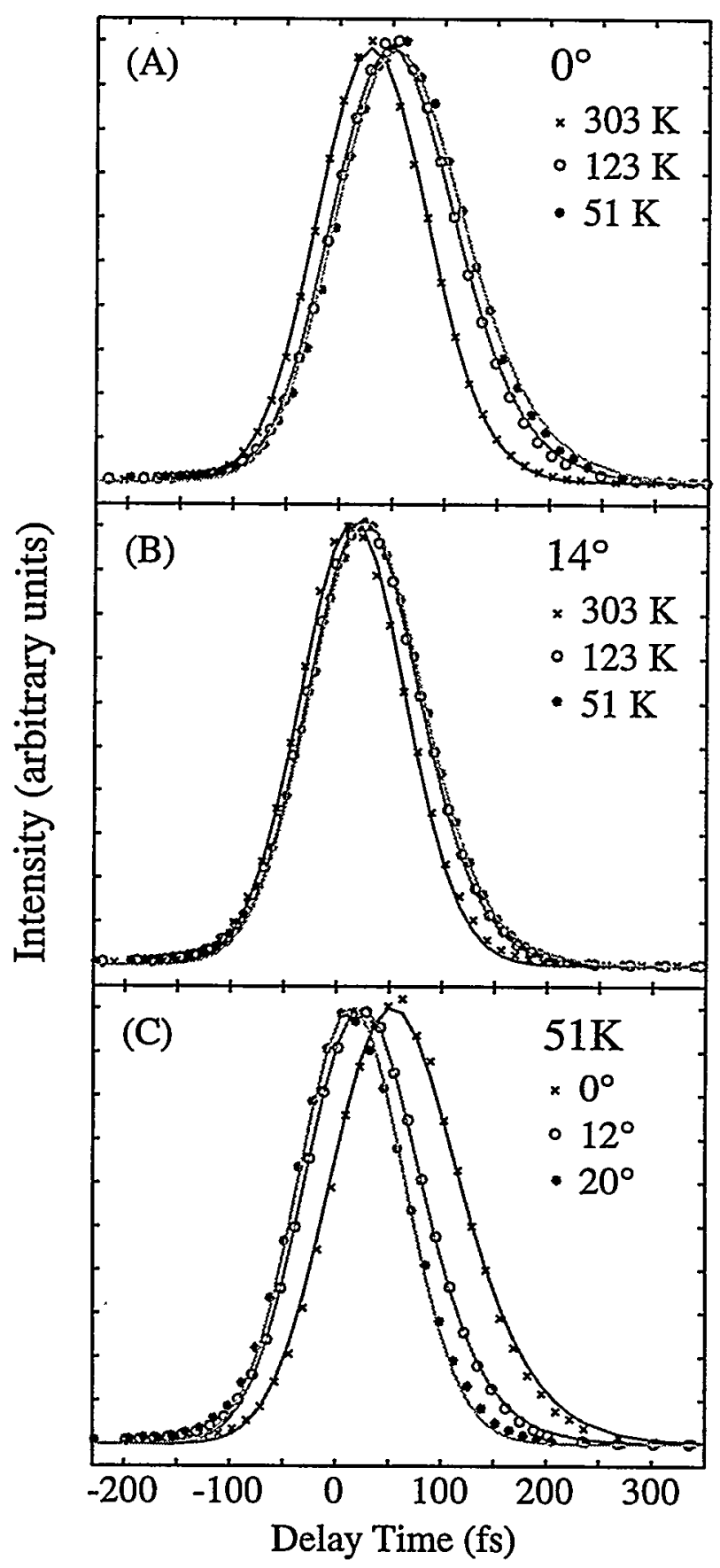

Figure 4.11: Time-resolved TPPE traces for the $n=1$ image state on $\operatorname{Ag}(111)$ for various temperatures and angles taken at UV/visible wavelengths of $300 \mathrm{~nm} / 600 \mathrm{~nm}$. Solid lines are fits obtained by convolving a Gaussian instrument function with the function of Eqn. 4.1 on page 55. Parameters are listed in Table 4.1 on the following page 


\begin{tabular}{rccccc|ccc}
\hline \hline & \multicolumn{2}{c}{$0^{\circ}(\mathrm{fs})$} & & \multicolumn{2}{c|}{$14^{\circ}(\mathrm{fs})$} & & \multicolumn{2}{c}{$51 \mathrm{~K}(\mathrm{fs})$} \\
\cline { 2 - 3 } \cline { 8 - 9 } & rise & decay & & rise & decay & & rise & decay \\
\hline $303 \mathrm{~K}$ & 11 & 22 & & 0.5 & 15 & $0^{\circ}$ & 32 & 33 \\
$123 \mathrm{~K}$ & 27 & 28 & & 0.1 & 25 & $12^{\circ}$ & 1 & 30 \\
$51 \mathrm{~K}$ & 32 & 33 & & 0.1 & 32 & $20^{\circ}$ & 0.1 & 16 \\
\hline \hline
\end{tabular}

Table 4.1: Fitting parameters for Fig. 4.11

TPPE experiments have been conducted at many angles and a plot of inverse lifetime as a function of $k_{\|}$should provide valuable information on which process is more important. It is, however, difficult to do a quantitative analysis in this system because the lifetimes are very short at high angles. A better system to test the prediction of $k_{\|}^{4}$ dependence would be the $\operatorname{Ag}(100)$ surface where lifetime as long as $180 \mathrm{fs}$ has been measured for the $n=2$ state [39]. 


\section{Chapter 5}

\section{Layer-by-Layer Evolution of}

\section{Electronic Structure}

The simple Coulomb interaction of an excess electron with a surface can be altered significantly by any factor which changes the dielectric susceptibility at the interface. Therefore electrons in image potential states can serve as sensitive probes of layer-by-layer changes in electronic structure and dynamics. Steinmann, Fauster, and coworkers used the fact that image state binding energies are pinned to the local work function [52] to observe the growth modes of $\mathrm{Ag}, \mathrm{Au}$ and $\mathrm{Co}$ on $\mathrm{Cu}(111)$ and $\operatorname{Pd}(111)[52,101,53]$ and have studied the evolution of metallic quantum wells for $\mathrm{Au} / \mathrm{Pd}(111)$ [101]. These studies examine the formation of an ultrathin interface of one free-electron material on another and were recently reviewed [8]. The effects of alkali metal overlayers have also been studied $[85,102]$. 
Aside from metallic adsorbates, the addition of dielectric adsorbates to a metal substrate was experimentally studied by several groups [8]. In general, image states can also exist outside a nonmetallic substrate and the image potential is reduced to

$$
V(z)=-\frac{\beta e^{2}}{4 z}, \quad \beta=\frac{\epsilon-1}{\epsilon+1} .
$$

where $\epsilon$ is the dielectric constant. This leads to a corresponding reduction in the binding energies. In fact, the existence of image states was first experimentally observed on the surface of liquid helium [14]. Recently, there have also been reports of the observation of image states on semiconductors $[103,104]$. For dielectric overlayers on a metal substrate, a somewhat smaller reduction of binding energy is expected. Changes of the effective mass or lifetime may occur as well. The electronic structure of the adsorbate might lead to the observation of non-image states. In many cases, the image states disappear after saturation of bare metal surfaces with diatomic molecular adsorbates [8]. However, Harris and coworkers showed that the modification of image states by the presence of physisorbed alkane and rare gas overlayers can be used to study electronic structure and dynamics at interfaces in layer-by-layer fashion $[69,70,81]$. 


\subsection{Previous Studies of Image Electrons at Metal-}

\section{Alkane Interfaces}

Several important results emerged from these initial TPPE experiments by the Harris group using picosecond lasers. First, image potential states persist, modified in energy and effective mass, in the presence of many physisorbed alkane and rare gas monolayers $[69,70]$. These layers are composed of either electronically inert atoms with closed shells or sigma-bonded hydrocarbons with relatively high-lying electronic excited states. The potential in the vacuum is still determined by induced polarization forces between the excess electron and the materials (metal and overlayer) comprising the surface $[20,105]$. However, overlayers composed of $\pi$-bonded molecular species such as alkenes, or molecules with attractive electron affinities in the gas phase, such as $\mathrm{C}_{6} \mathrm{~F}_{12}$, destroy the simple image potential Rydberg structure even for monolayer coverages. These latter effects are not yet understood.

In general, thicker layers of alkane molecules on the surface result in decreased image potential electron binding energies. This is consistent with more efficient screening of the metallic image potential by a thicker dielectric layer, and implies that the electron resides largely outside the layer in the attractive image-like potential well associated with the dielectric polarizability of the insulator slab. These data can be at least qualitatively described by fairly simple dielectric models. Binding energy data of this type as a function of layer thickness and layer composition help determine a 
model of the potential and hence the electron wavefunction at the interface. However, a more revealing test of where the electron probability density is concentrated, i.e. near the metal or out at the dielectric-vacuum interface, would be a lifetime measurement. TPPE data with femtosecond time resolution for a variety of overlayers is needed to resolve these questions.

\subsection{Femtosecond Results for n-Alkanes/Ag(111)}

Figure 5.1 shows the TPPE binding energy spectra at $0^{\circ}$ emission upon the adsorption of $\mathrm{n}$-heptane using $300 \mathrm{~nm}$ pump and $600 \mathrm{~nm}$ probe wavelengths. The monolayer spectrum in Fig. 5.1 clearly shows the first three member of the image potential Rydberg series. The binding energies are sensitive to the layer thickness, becoming less bound with increasing coverage. This change in binding energy clearly corresponds to a layer-by-layer evolution of the surface potential.

Even more dramatic changes are seen in the electron lifetimes. Figure 5.2 shows the femtosecond population dynamics for the $n=1,2$, and 3 states of the monolayer shown in Fig. 5.1. We also observe a small background signal at $t=0$ in the region of energies corresponding to -0.3 to $+0.2 \mathrm{eV}$ in Fig. 5.1 when using a UV pump wavelength near the photoelectric threshold. Because a longer time range was used for monolayer dynamics scans (1500 fs full range) than for the clean surface scans (700 fs full scans), a coarser time step was used for the monolayer scans. Within

the limitation of the coarser time steps ( $33 \mathrm{fs}$ versus $13 \mathrm{fs}$ ), the dynamics of the 


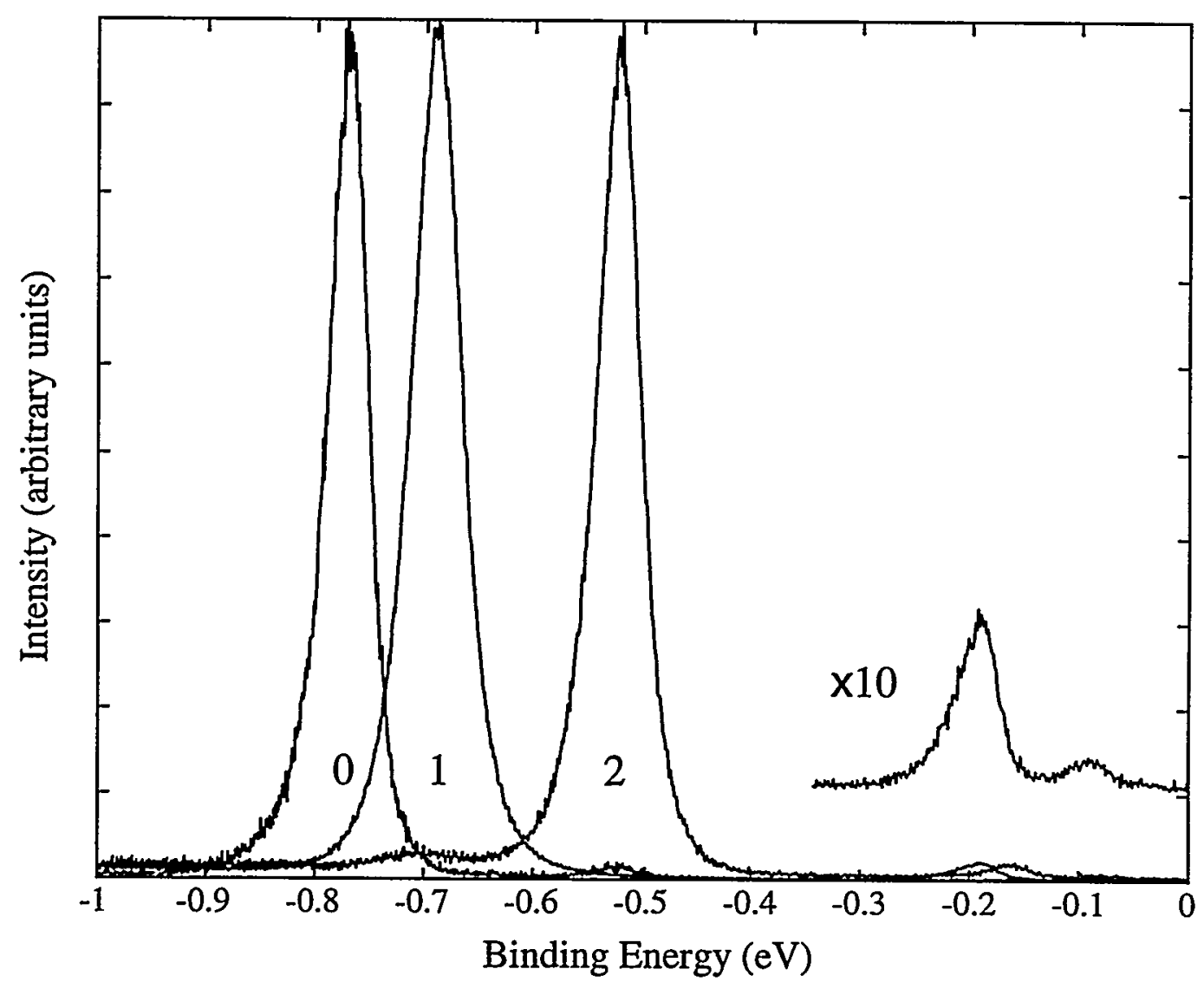

Figure 5.1: TPPE Spectra for the image states showing their change in binding energy as a function of $\mathrm{n}$-heptane layer thickness on $\mathrm{Ag}(111)$, taken at a pump-probe delay of $160 \mathrm{fs}$. Numbers indicate number of layers. The $n=2,3$ state region is magnified 10 times for the monolayer. 


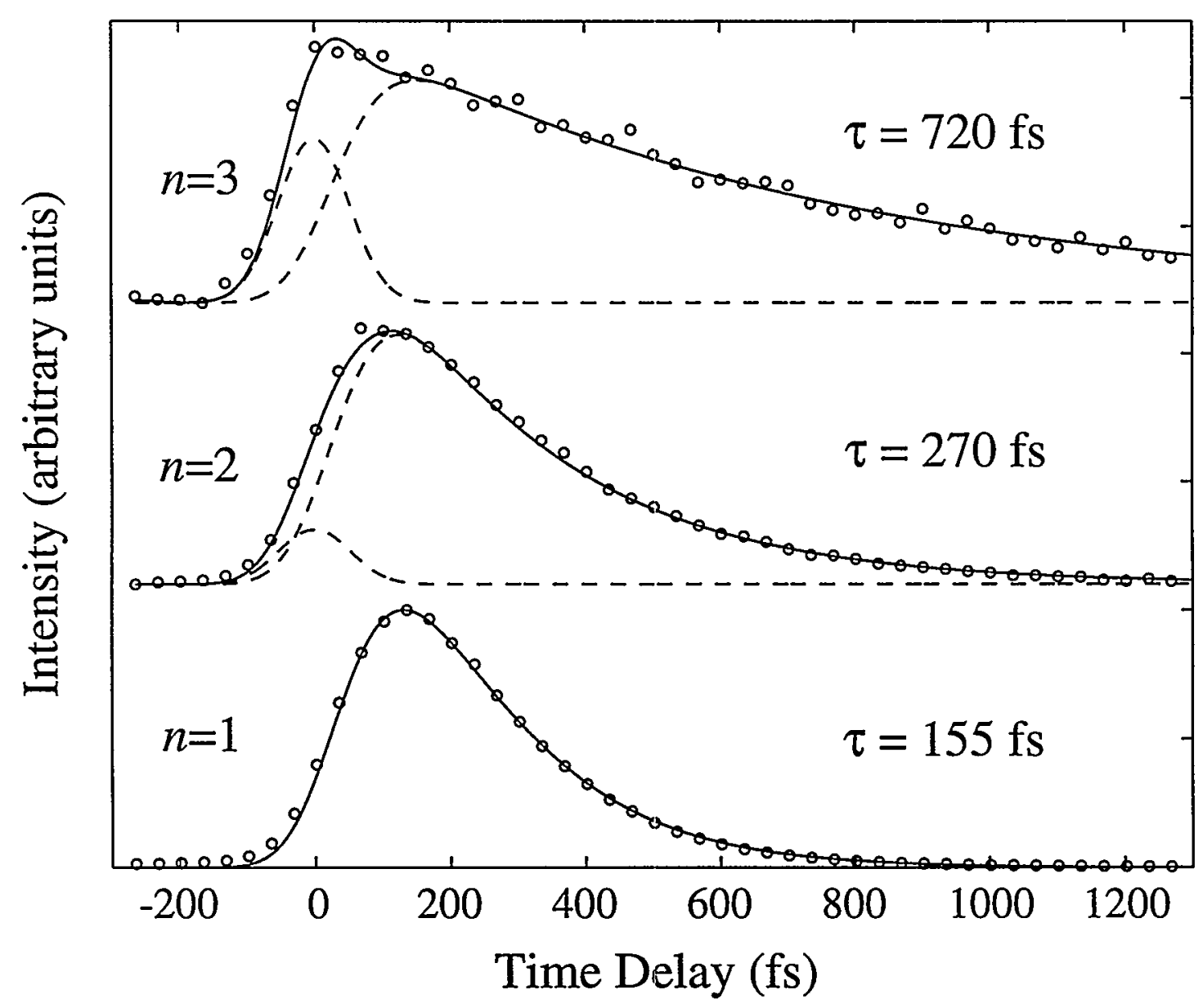

Figure 5.2: The femtosecond population dynamics of the $n=1,2$, and 3 states for a monolayer of $n$-heptane $/ \operatorname{Ag}(111)$. The component at time zero in the $n=2$ and 3 data has similar dynamics to the instrument function (see text) and is tentatively assigned to TPPE from the valence band edge. The data are modeled by convolving the instrument function shown in Fig. 4.7 with the sum of a single exponential decay and the function of Eqn. 4.1. 
near-threshold photoemission are similar to those of the occupied surface state on the bare metal, which serves as our instrument function. Time-delay scans with finer time steps on the monolayer sample should clarify the dynamics of this spectral feature. We speculate that this near-threshold photoemission is due to a UV+visible excitation from the valence band edge. It may also be resonant with very short-lived states in the bulk conduction band. Although the origin of this emission is uncertain, the dynamics are in any case similar to those of the instrument function within the resolution of these data and can be included in the model for the data as a feature of known width, adding only an amplitude parameter to the fit.

Thus the $n=2$ and 3 decay curves of Fig. 5.2 have been fit by convolving the instrument function with a model comprising Eqn. 4.1 plus a 1 fs single exponential decay. The dashed lines in Fig. 5.2 show the individual contributions to the fit; the decay times listed on the figure correspond to $k_{2}^{-1}$ in Eqn. 4.1. The image state lifetimes for the monolayer case become progressively longer with higher quantum number, as is the case for $\operatorname{Ag}(100)$ where the whole Rydberg series is in the projected bulk band gap. As already noted, such is not the case for bare $\operatorname{Ag}(111)$. Thus the band structure effect is no longer the dominant factor in determining the quantum number dependence of the lifetimes in the presence of a monolayer.

For bilayer n-heptane/ $\operatorname{Ag}(111)$, the $n=1$ lifetime is two orders of magnitude longer than the bare surface value. The dynamics of the $n=1$ surface electron populations on bare $\mathrm{Ag}(111)$, monolayer n-heptane $/ \mathrm{Ag}(111)$, and bilayer n-heptane/ $\mathrm{Ag}(111)$ 
are plotted together in Fig. 5.3 to illustrate the signal-to-noise ratio of the data and highlight the drastic difference in the dynamics. In sharp contrast to the monolayer result, both the bilayer $n=2$ and 3 lifetimes are once again shorter than for $n=1$. The lifetime data are summarized in Table 5.1, where the boldface numbers represent experimental values. 


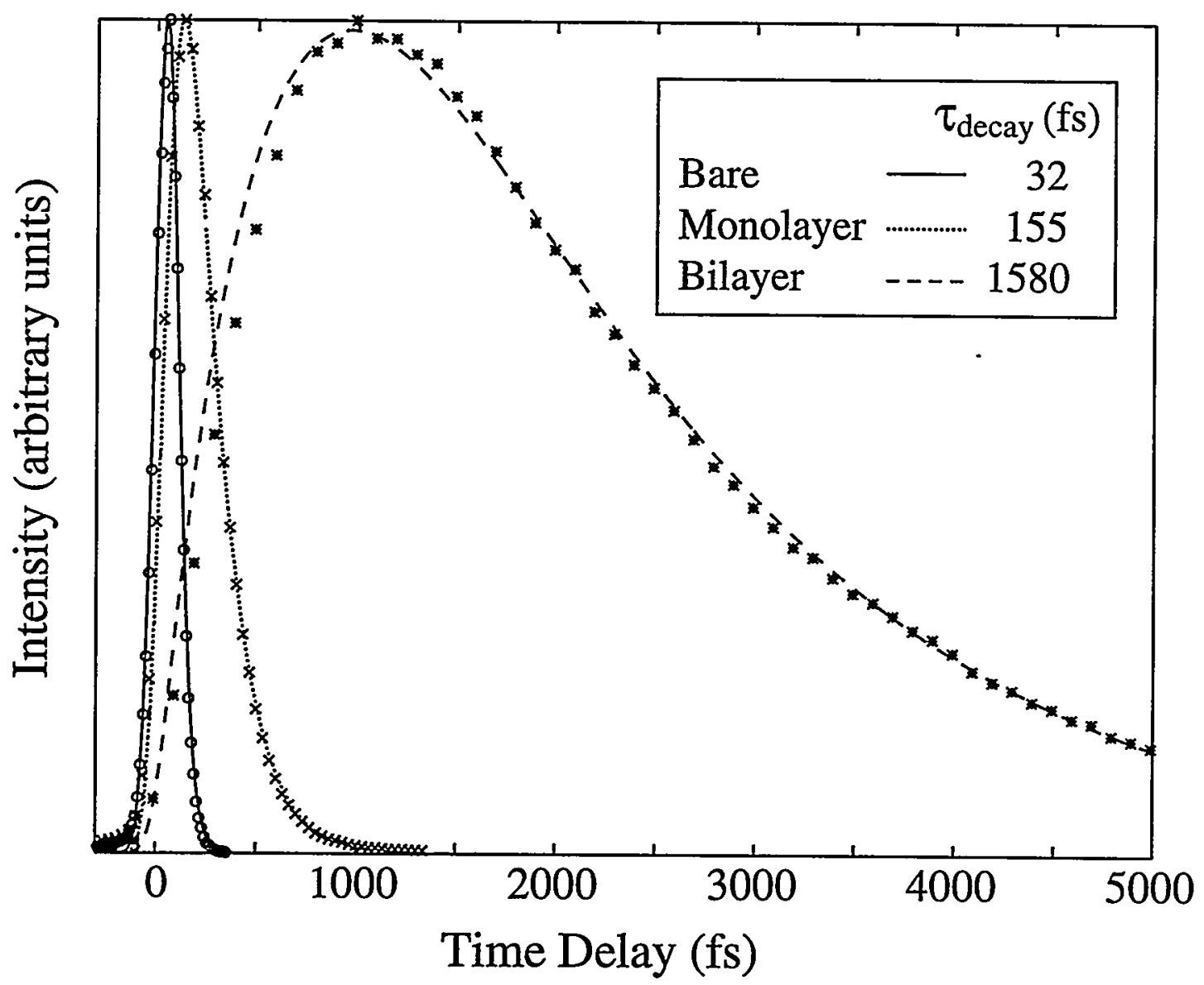

Figure 5.3: The femtosecond population dynamics in the $n=1$ state for bare $\mathrm{Ag}(111)$, monolayer n-heptane $/ \operatorname{Ag}(111)$, and bilayer $n$-heptane/ $\operatorname{Ag}(111)$. The data are fit by a rise time and a decay time according to the model in Eqn. 4.1. The decay times are shown in the figure. The rise times are 25 fs for the clean surface, 80 fs for the monolayer, and $700 \mathrm{fs}$ for the bilayer. 


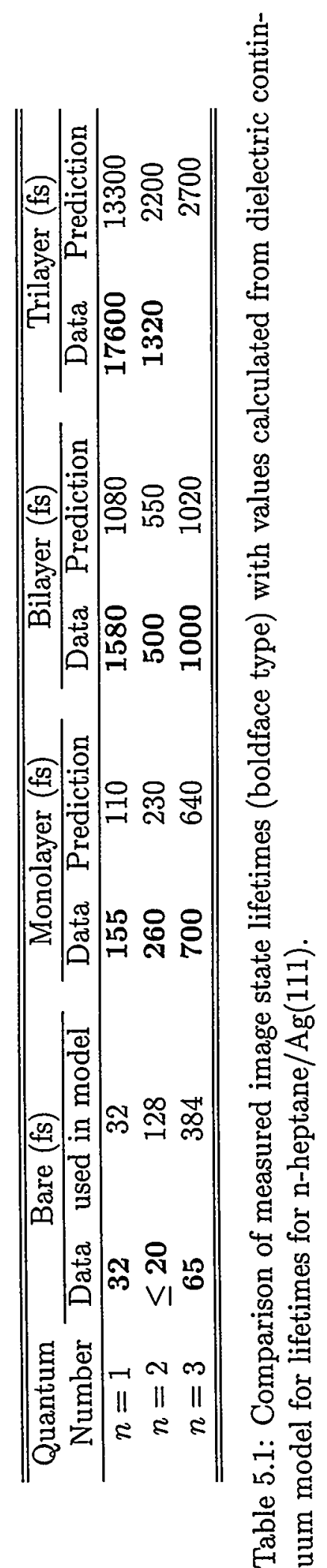




\subsection{Analysis and Discussion}

The first interesting result of this work has already been mentioned: adsorption of a monolayer not only dramatically increases the image state lifetimes but also alter the progression of lifetime with quantum number $n$ as shown in Table 5.1. The trend of lifetimes are remarkably similar to theoretical predictions and measurements for bare $\operatorname{Ag}(100)$ where image states are not degenerate with the projected bulk conduction band and the lifetime is a strong and monotonic function of $n$. This behavior suggests that the excess electron states at the interface are no longer strongly coupled to the bulk conduction band.

The likely explanation for this effect is that the higher quantum states drop into the gap due to the work function shift on adsorption, so that the lifetimes vary as on bare $\operatorname{Ag}(100)$. The work function shift associated with the adsorption of the monolayer moves the vacuum level (and thus the image state series) $\sim 430 \mathrm{meV}$ closer to the Fermi level, as determined by threshold UPS. This will move the $n=2$ state into the gap, leaving it $\sim 55 \mathrm{meV}$ below the conduction band edge. However, uncertainties in the absolute work function of the substrate, the work function shift due to adsorption, and the exact location of the bulk conduction band edge render it difficult to say for certain if the $n=3$ state is degenerate with the bulk conduction band. Our best estimate with current data and literature values [8] is that $n=3$ does remain $\sim 40 \mathrm{meV}$ above the bulk conduction band edge in the presence of the physisorbed monolayer. 
If $n=3$ is still degenerate with the bulk conduction band, then the ability of the monolayer to mitigate the effect of degeneracy with the conduction band might be due to the significant modification of the surface potential by the adlayer. Solid and liquid alkanes generally possess a bulk electron affinity repulsive by a few tenths of an electron volt [106]. This repulsive interaction will serve to weaken the coupling of the electron to the bulk states, even for a monolayer where the electron affinity may be smaller than the bulk value. and the effective potential near the surface may not be high enough to constitute an actual barrier. This effect might allow the image potential electrons to assume their "in the gap" lifetimes in spite of being isoenergetic with the projected bulk conduction states. Similar arguments has been applied to compare the $n=3$ lifetimes of cyclohexane and Xe monolayers [64].

Secondly, the major result of these experiments is evident from examining the $n=1$ lifetimes in Table 5.1. The approximately exponential increase in lifetime indicates that increasing the adlayer thickness presents the image electron with a wider barrier through which it must tunnel in order to decay back into the metal. The existence of the tunneling barrier is consistent with the repulsive electron affinity of the longer chain n-alkanes in the condensed phase.

Thirdly, although the $n=2,3$ lifetimes also increase with coverage, the increase is less dramatic than $n=1$. This indicates that the $n=2$ and 3 states are less influenced by the increasing width of the tunneling barrier presented by the layer because they are 500 to $600 \mathrm{meV}$ higher in energy than the $n=1$ state. This difference in the effect 
of the tunneling barrier for the various quantum levels is clearly seen in the bilayer and trilayer columns. The lifetime is no longer a monotonic function of $n$, with the $n=1$ lifetime being the longest. To illustrate the essential physics of the preceding paragraphs, we compare our data to the results of model calculation as shown below.

\subsection{Dielectric Continuum Model}

To model image states perturbed by the presence of the dielectric layer, we have employed the well-established dielectric continuum model first presented by Cole [107]. The electron is free-electron-like in the direction parallel to the surface, but bound to the surface in the perpendicular direction by a one-dimensional model potential. In the vacuum region, the electrostatic energy of a point charge outside a metal covered with a continuum dielectric overlayer of thickness $d$ and dielectric constant $\epsilon$ is given by

$$
V_{\text {out }}(z, d)=\frac{-\beta e^{2}}{4(z-d)}+\frac{\left(1-\beta^{2}\right) e^{2}}{4 \beta} \sum_{n=1}^{\infty} \frac{(-\beta)^{n}}{z-d+n d}, \quad z>d
$$

The first term is the attractive image potential of an excess electron outside an infinite dielectric. The second term describes the interaction of the electron with the metal modified by the presence of the dielectric layer. The infinite series can be interpreted as sum of successive corrections due to image charges inducing image charges. Inside 
the dielectric, the potential can be taken as

$$
V_{\text {in }}(z)=-\frac{e^{2}}{4 \epsilon z}+V_{0}, \quad z<d
$$

where the first term is the metal image potential screened by the presence of the dielectric layer, and $V_{0}$ is the bulk electron aflinity (conduction band minimum with respect to the vacuum level) of the dielectric. Therefore, the use of $\epsilon$ in the potential describes dielectric effects: The presence of a dielectric layer adds a new attraction between the electron and the adlayer but weakens the interaction between the electron and the metal. The appearance of $V_{0}$ as an additive constant to the potential provides the simplest description of the overlayer band structure. Since $V_{\text {in }}$ and $V_{\text {out }}$ are not valid near $z=0$ and $z=d$, respectively, near the metal $V_{\text {in }}$ is cut off at the Fermi level, and a cutoff parameter $b$ is employed at the layer-vacuum interface so that $V_{\text {out }}$ is chosen to be a constant for the range $d<z \leq d+b$.

We have obtained the most complete set of layer-resolved binding energy data for n-octane/ $\operatorname{Ag}(111)$ and have compared these binding energies to the eigenenergies predicted by Cole's model potential as a test of its validity. The Schrödinger equation is solved by numerically diagonalizing the Hamiltonian using a discrete variable representation algorithm [108] with the boundary conditions of vanishing wavefunction at $z=0$ (no penetration into the metal) and at infinity. [Thus this model cannot include any band structure effects. However, we have argued above from the lifetime 
data that band structure effects are relatively minor for the monolayer; thus we proceed under the approximation that the potential outside the metal has the dominant effect.] We do not have literature values for $\epsilon$ and $V_{0}$ of crystalline n-heptane and noctane. To go from liquid data [109] to the solid [110], the Clausius-Mossotti relation was used and the dielectric constants were determined to be $\approx 2.3$. By comparison with other n-alkanes, the electron affinities in our case should be about $0.2 \mathrm{eV}$ at the freezing point [106]. The potential for the bilayer case with $\epsilon=2.0, V_{0}=0.2 \mathrm{eV}$, and $b=1 \AA$ is shown in Fig. 5.4. The calculated binding energies of the $n=1$ state using this set of parameters are in good agreement with experiment as shown in Fig. 5.5. From the model potential (Fig. 5.4) and the wavefunctions (Fig. 5.6 on page 82), we see that the electrons tend to be excluded from the layer due to the potential barrier set up by the repulsive electron affinity of the alkane layer. As the layer thickness increases, the electron probability density remains largely outside at the layer-vacuum interface (Fig. 5.6). The coupling to the metal decreases and electrons become less bound.

We will now examine the effect of the tunneling barrier on image state lifetimes. On bare metal surfaces, the lifetime $n^{3}$ dependence can also be thought of as being due to the $n^{3}$ variation of the classical period of motion in the potential well, which constitutes an "attempt rate" for penetration into the bulk of the metal substrate. Electrons in the higher quantum state travel to a longer distance, experience a flatter part of the Coulomb potential and a softer restoring force. Therefore, electrons spend 


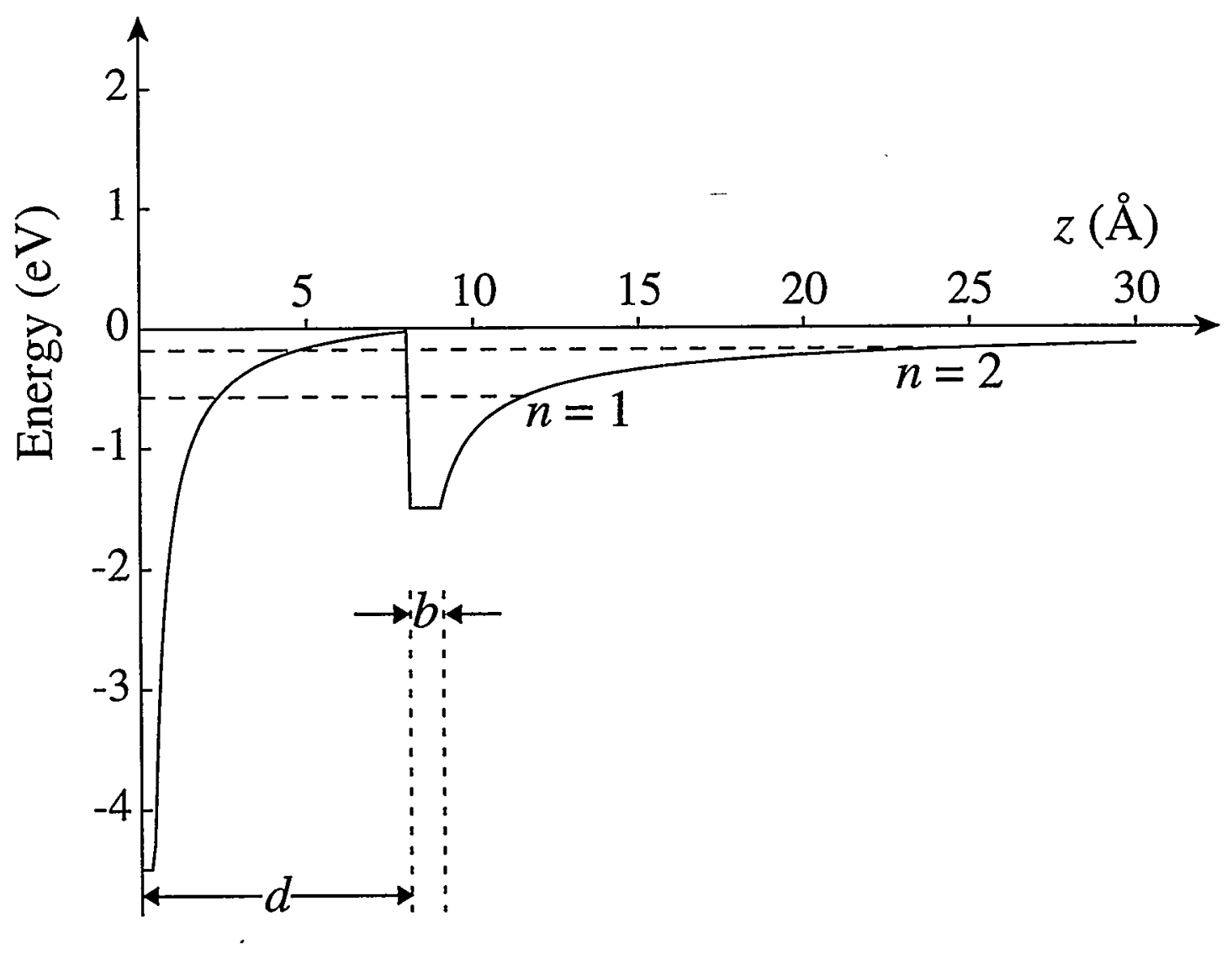

Figure 5.4: Model potential for a $d=8 \AA$ dielectric overlayer on a metal surface, corresponding to a bilayer of a n-octane. Dielectric continuum model for a bilayer of $\mathrm{n}$-octane. A potential cutoff is imposed at the metal-layer and layer-vacuum interface to avoid the singularity in the potential. 


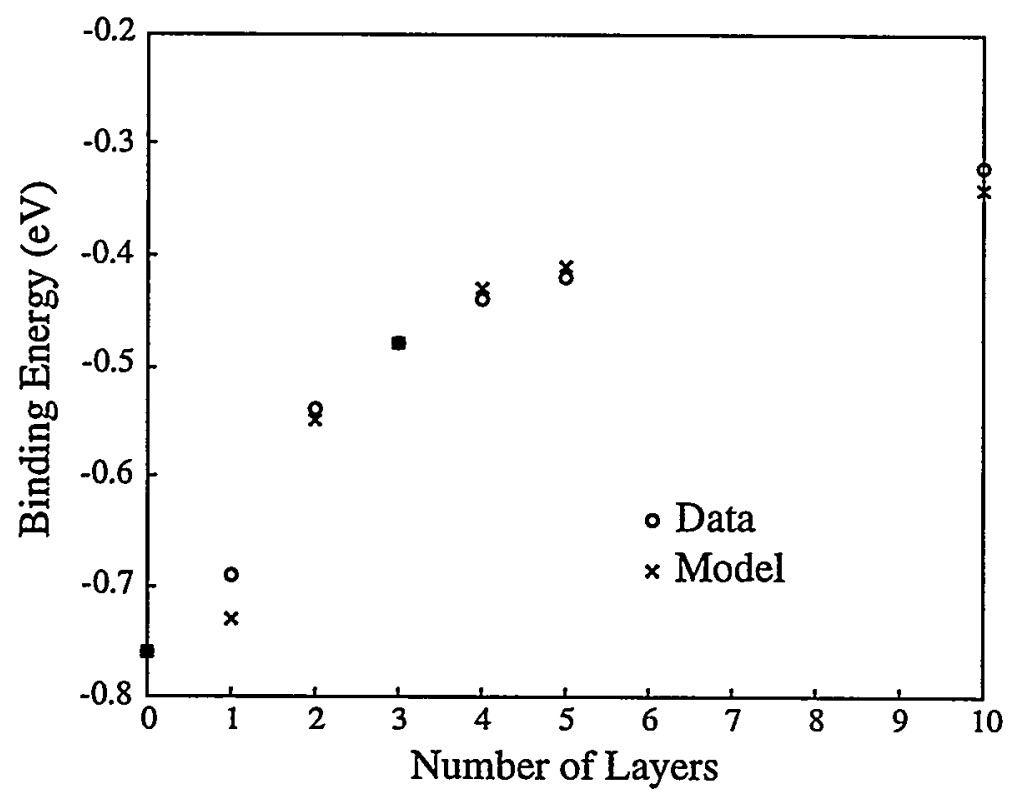

Figure 5.5: Experimental binding energies ( $\circ$ ) of the $n=1$ states as a function of n-octane coverage, and a comparison to the results of a dielectric continuum model $(x)$. The parameters $\epsilon=2.0, V_{0}=+0.2 \mathrm{eV}$, and $b=1 \AA$ were used in Eqns. 5.2 and 5.3 on page 78 .

\begin{tabular}{c|rrrrrrr}
\hline \hline Number of layers & 0 & 1 & 2 & 3 & 4 & 5 & 10 \\
\hline Approx. thickness $(\AA)$ & 0 & 4 & 8 & 12 & 16 & 20 & 40 \\
\hline Binding Energy, data $(\mathrm{eV})$ & -.76 & -.69 & -.54 & -.48 & -.44 & -.42 & -.32 \\
Eigenenergy, model $(\mathrm{eV})$ & -.76 & -.73 & -.55 & -.48 & -.43 & -.41 & -.34 \\
\hline \hline
\end{tabular}

Table 5.2: Data and calculated values for Fig. 5.5. 


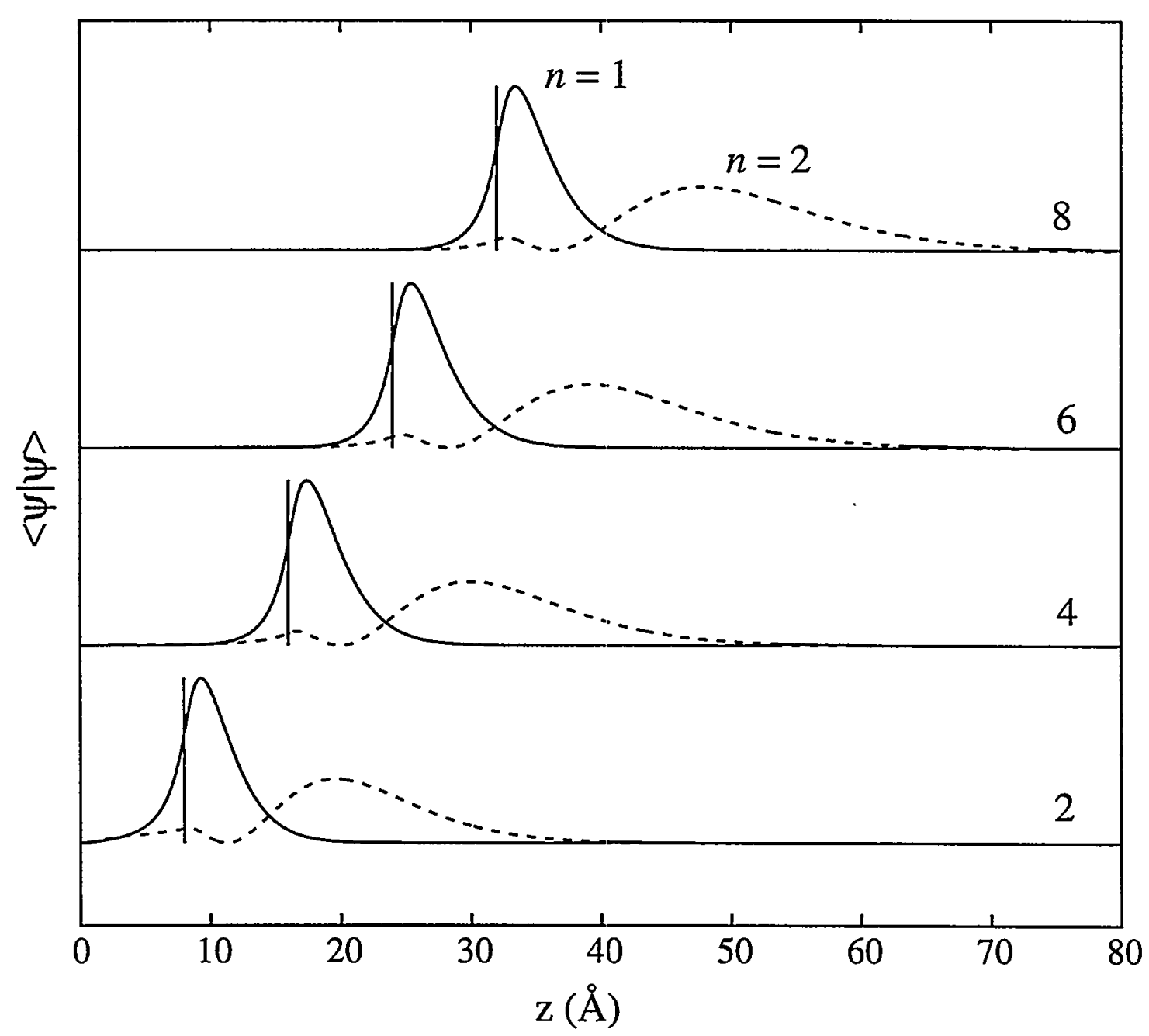

Figure 5.6: The probability density for the $n=1$ and 2 image states for bilayer noctane/ $\mathrm{Ag}(111)$ calculated from the dielectric continuum model The solid and dashed lines are for the $n=1$ and 2 states, respectively. The vertical lines indicates the layervacuum interface. Numbers indicate number of layers. 
more time at the outer turning point and less time near the metal. The lower attempt rate makes their lifetimes longer. With the presence of electron repulsive overlayer, the probability of tunneling through the effective potential barrier determines how likely a decay attempt is successful (Fig. 5.4). The lifetime of the electrons is then determined by both factors [107]:

$$
{ }^{\top} \tau=T_{\mathrm{cl}} P^{-1}
$$

where $T_{\mathrm{cl}}$ is the inverse of the attempt rate, equal to the period of motion between the outer classical turning point and the barrier, and $P$ is the tunneling probability. Using the WKB approximation, Cole determined expressions for $P$ :

$$
\begin{gathered}
P=\mathrm{e}^{-2 J} \\
J=\int_{z_{0}}^{d} d z\left(\frac{2 m\left[V_{\text {in }}(z)-E\right]}{\hbar^{2}}\right)^{1 / 2} \\
=\left(\frac{8 m\left(V_{0}-E\right) d^{2}}{\hbar^{2}}\right)^{1 / 2}\left\{\left(1-y^{-2}\right)^{1 / 2}-y^{-2} \ln \left[y+\left(y^{2}-1\right)^{1 / 2}\right]\right\}
\end{gathered}
$$

where $z_{0}$ is the classical turning point on the metal side of the adlayer, and $y^{2}=$ $4 d \epsilon\left(V_{0}-E\right) / e^{2}$. The quantity $y$ should be greater than one, otherwise the barrier is completely eliminated and this approach is invalid. The quantity $T_{\mathrm{cl}}$ is given by an 
integral over the classically allowed range:

$$
T_{\mathrm{cl}}=2 \int_{d}^{z_{m}} d z\left(\frac{m}{2\left[E-V_{\text {out }}(z)\right]}\right)^{1 / 2}
$$

where $z_{m}$ is the classical turning point on the vacuum side. He also determined the analytical expressions for the upper and lower bound of $T_{\mathrm{cl}}$, with upper bound taking the form of

$$
T_{\mathrm{cl}} \approx \frac{1}{2}\left(\frac{2 m\left(z_{m}-d\right)}{V_{\text {out }}^{\prime}\left(z_{m}\right)}\right)^{1 / 2}
$$

where the prime denotes differentiation. It is clear from Eqns. 5.9 and 5.6 that if the classical turning point is far away from the metal and if the slope of the potential (the classical force) at that point is small, then the wavepacket will spend most of the time out in the potential well. Then $T_{\mathrm{cl}}$ will be long and the attempt rate for tunneling will be relatively low. If the electron affinity is more repulsive with respect to the eigenenergy and the layer is thick, the electron has less chance to tunnel through the barrier and $P^{-1}$ will be high. The lifetime will be lengthened in both cases.

Since the binding energies and lifetimes for n-octane are similar to those for $\mathrm{n}$ heptane, and since their bulk dielectric constants and electron affinities are similar, we take the liberty of using the model potential parameter above to carry out the lifetime calculation for the $\mathrm{n}$-heptane/ $\mathrm{Ag}(111)$ data presented above. To apply the above lifetime model to our system, several modifications are necessary. First, Cole's 
model was not intended to calculate image electron decay times on the bare metal surface; the overlayers envisioned in that paper were highly repulsive rare gases, where the tunneling time through the layer would dominate and the decay time once the electron was at the metal surface could be taken as zero. Therefore we have implemented Cole's model for these samples by using the measured bare surface lifetimes as the basic decay times and using Cole's predictions to scale them for the attempt rate and barrier tunneling effects. Since there is no adlayer barrier due to a dielectric film on the bare surface, we set $P^{\text {bare }}=1$ for the bare surface and assume that the lifetime is a measurement of its $T_{\mathrm{cl}}$ multiplied by a factor including all possible decay processes into the metal. We thus scale the lifetime for the bare surface by two factors to approximate the lifetime on the adlayer:

$$
\tau^{\text {adlayer }}=\tau^{\text {bare }}\left(\frac{T_{\mathrm{cl}}^{\text {adlayer }}}{T_{\mathrm{cl}}^{\text {bare }}}\right)\left(\frac{P^{\text {bare }}}{P^{\text {adlayer }}}\right)
$$

For calculating the effect of the the overlayers on the $n=2$ and 3 lifetimes, it is clearly not appropriate to use the bare surface lifetimes on $\mathrm{Ag}(111)$. These lifetimes are anomalous due to the fact that these states are degenerate with the bulk conduction band. Since the bulk conduction band is not a dominant factor for the monolayer as discussed above, we adjust the bare surface $n=1$ lifetime for the expected progression of lifetime with $n$ in the absence of degeneracy. Theory $[18,36]$ predicts $n^{3}$ lifetime scaling for states $n \geq 5$, and somewhat less than $n^{3}$ for lower quantum state, with 
$\tau_{n=2} / \tau_{n=1} \approx 4$ and $\tau_{n=3} / \tau_{n=1} \approx 12$. Thus we use these factors in the calculation.

Also, Eqn. 5.5 on page 83 is an valid approximation to the tunneling probability when $z_{0}$ and $d$ are widely separated. For the $n=2$ and 3 states that are close to the top of the barrier, this condition is not fulfilled. Therefore, the exact WKB expression

$$
P=\frac{1}{1+\mathrm{e}^{2 J}}
$$

is used in the calculation. Finally, in the case of monolayer, the eigenenergies of the $n=2$ and 3 states are slightly above the barrier, i.e., $y<1$ and the two turning points are located in the complex plane $[111,112]$. A method that is frequently used to deal with this situation and is applied here is to extrapolate $J$ from the known region of $E<V$ to the region of $E>V$ [113]. The experimentally determined and model-predicted lifetimes of the $n=1,2$, and 3 states for mono-, bi-, and trilayer n-heptane/ $\operatorname{Ag}(111)$ are listed in Table 5.1 on page 74.

\subsection{Implications and Comparison to Electron}

\section{Attractive Overlayers}

The application of Cole's model to the mono- and bilayer extremes stretches its assumptions, but the correlation between the model predictions and the TPPE data for binding energy and lifetime support the conclusion that it does correctly identify 
the important factors of barrier penetration and attempt rate. Several further tests of the model and the physical ideas that it implements are obvious. First, a cleaner comparison with Cole's model could be made for dielectric layers grown on $\mathrm{Ag}(100)$, where all members of the image potential Rydberg series are in the projected bulk band gap for both the clean and adlayer-covered surfaces.

The almost exponential lengthening of image state lifetimes in the presence of a few molecular layers strongly suggests that the image potential electron probability density is significantly pushed out from the metal surface. We note that the presence of the tunneling barrier is, of course, not dependent on the electronic structure of the substrate. The entire image potential Rydberg series would be degenerate with the bulk conduction band for $\mathrm{Au}(111)$, where linewidth measurements point to an $n=1$ lifetime of a few femtoseconds $[8,114]$. The tunneling barrier presented by an alkane adlayer should allow this broad $n=1$ surface resonance of the bare metal to develop into a quasi-stationary, true surface state at some thickness.

The tunneling barrier presented by an adlayer with repulsive electron affinity will be present for any metal substrate and should be considered in the interpretation of some experiments involving photoinitiated charge transfer. In particular, n-alkanes are sometimes used to isolate other adsorbates from direct electronic interaction with a metal surface or used to systematically vary the distance between the surface and an admolecule. The significant increase in image potential electron lifetimes reported here show that the use of such a spacer layer will weaken electronic coupling across 
the interface.

Systems with attractive electron affinities such as neopentane or Xe should give very different results from those with repulsive electron affinities such as the n-alkanes because electrons tend to be drawn into the layer. These systems offer the opportunity to observe the dynamics of electrons as the adlayer conduction band develops in layerby-layer fashion. The static TPPE study of multilayer $\mathrm{Xe} / \mathrm{Ag}(111)$ [86] indicates quantum confinement effects in the xenon slab. The attractive electron affinity $\left(V_{0}=\right.$ $-0.5 \mathrm{eV}$ ) of the Xe slab provides a shallow quantum well, bounded by the bulk band gap of the substrate on one side and the image potential on the other side. As the Xe layer thickness increases from 1 to 9 layers, the $n=2,3$ image states of the bare metal, with binding energy originally being above the bulk Xe conduction band minimum, evolve into quantum well states of the layer, and become more bound. Just like a particle in a box, this lowering in the electron energy is due to less quantum confinement. In contrast, the $n=1$ image state of the bare metal, with its binding energy originally being below the Xe conduction band minimum, becomes less bound with increasing layer thickness. This coverage dependence is characteristic of an image state outside an dielectric layer, similar to the behavior of alkane overlayer.

Although the dielectric continuum model employed above works quantitatively for alkane overlayer, a variation of this model $[115,86]$ works only qualitatively for Xe overlayer [86]. It overestimates the binding energies for all three states, but it does reproduce the major trends in the data. The reason for its failure is that treating the 
metal as a hard wall and ignoring the band dispersion of the layer are only valid for electron repulsive overlayers where the electrons are decoupled from the metal and reside largely outside the layer. In the case of $\mathrm{Xe}$, a simple model which takes into the band structures of the metal and the overlayer, as well as the image potential in the vacuum, has been developed by McNeill et al. [86, 64]. The details of the model calculation for the electron binding energies and lifetimes will be presented in the thesis of Jason McNeill [116]. In this model, the substrate bands are treated within the nearly-free-electron two-band model [19] (Section 2.2). The overlayer band is treated within the effective mass approximation [117] to include the effect of electron affinity as well as the band dispersion. The latter is of particular importance because the effective mass of the bulk Xe conduction band is $\sim 0.35 m_{\mathrm{e}}$ [118], significant less than that of the free electron. The image potential in the vacuum is taken from Eqn. 5.2. Good agreement with the binding energy data is achieved [86].

Under this formalism, lifetime predictions can be obtained from the calculated wavefunctions by assuming that the penetration of the electron into the metal is a measure of the electron's coupling to the bulk. Femtosecond time-resolved measurements on 1-6 layers of $\mathrm{Xe}$ on $\mathrm{Ag}(111)$ [64] showed that the $n=2,3$ electron lifetime exhibits oscillation as a function of Xe layer thickness. This coverage dependence is very different from the alkane case, where lifetime increases monotonically with layer thickness. This behavior can be understood from the spatial distribution of the electron as a function of coverage [64]. At low coverage, the electron still behaves like 
an image electron, residing largely at the layer-vacuum interface. The adsorption of additional layers weakens the coupling to metal and increases electron lifetime. As the layer reaches a certain thickness, it is energetically favorable for the electron to move inside the layer. The probability density inside the layer increases, and the penetration into the metal increases, therefore the lifetime decreases. When the layer gets even thicker, the classical round-trip time within the layer increases, the attempt rate for decaying back into the metal decreases, and the lifetime increases again. Therefore, the oscillations in the lifetime for $\mathrm{Xe} / \mathrm{Ag}(111)$ is the signature of electrons moving inside the interfacial quantum well. This behavior is successfully accounted for by the model calculations of McNeill et al. [64]. The comparison between the results for alkane and Xe overlayers clearly shows that the dynamics of excess electrons are largely determined by the electron affinity of the adsorbates. 


\section{Chapter 6}

\section{Lateral Motion of the Interfacial}

\section{Electrons: Dynamic Localization}

Polarization interactions and the localization of charge carriers in condensed media continue to represent a challenge for theoretical descriptions of the interaction of a carrier with its environment. The need for a detailed understanding of charge localization is also heightened by its importance in determining the electronic and optical properties of applied materials and in the development of new materials.

The delocalization and localization of electrons is fundamentally interesting, especially at material interfaces where lower dimensionality may result. Electrons in a number of physical systems exhibit two-dimensional behavior $[14,119,120]$. The spatial extent and the transport properties of electrons supported on a liquid helium surface have been the subject of extensive experimental and theoretical studies. In- 
teresting features such as the Coulomb crystallization on bulk helium [121] and the polaronic transition on a helium film [122] were experimentally observed. However, the problem of electron localization at atomically thin dielectric-metal interfaces remains poorly understood owing to complexities associated with the morphology of the interface, the electronic structure of the substrate and the overlayer, and the coupling to the vibrational modes of the composite system. This chapter investigates how the transition from delocalized to localized electronic behavior near the metal-dielectric interface occurs dynamically.

\subsection{Previous Studies with Picosecond Lasers}

Unlike the core electrons tightly bound to atomic nuclei, valence electrons in metals are normally delocalized to a greater or lesser degree as manifested by how much the bands disperse. One important phenomenon that happens near an interface is that delocalized electrons can become localized in one or more directions $[9,10,11,29,54,123]$. Image potential electrons are held close to the surface by the image potential but can move relatively freely parallel to it. The motion of the electron along the interface and how it is affected by the substrate and the presence of overlayers can be directly studied by angle-resolved TPPE. For a bare metal substrate, when the lateral symmetry is broken by introducing steps on the surface, evidence for an isolated step-induced image state was reported, where the electron movement perpendicular to the steps was believed to be confined by the potential 
trough at the step edge [54]. In the case of dielectric overlayers, the static angleresolved TPPE results for excess electrons on $\mathrm{Xe} / \mathrm{Ag}(111)$ [86] and alkane/ $\mathrm{Ag}(111)$ [81] are summarized as follows.

At the $\mathrm{Kr}$ and $\mathrm{Xe} / \mathrm{Ag}(111)$ interfaces, an excess electron remains delocalized for all coverages [86]. For both the $n=1$ and $n=2$ states of Xe overlayers, the effective mass goes from that of a free electron at monolayer coverage $\left(0.95 \pm 0.1 m_{\mathrm{e}}\right.$ for $\left.n=1\right)$ to significantly less than that of a free electron $\left(0.6 \pm 0.2 m_{\mathrm{e}}\right.$ for $\left.n=1\right)$ at four layers. The dispersion data for one to two layers of $\mathrm{Kr}$ is similar to that of $\mathrm{Xe}$. The decrease of effective mass towards the literature values for the bulk $\mathrm{Kr}$ and $\mathrm{Xe}$ conduction bands (0.42 and $0.35 m_{\mathrm{e}}$, respectively [124]) demonstrates the influence of the overlayer band structure. The low effective mass and electron delocalization at these heavy rare-gasmetal interfaces is consistent with the fact that free-electron conduction with electron mobilities of typically a few thousand $\mathrm{cm}^{2} / \mathrm{V}$ is found in all the heavier condensed rare gases [124]. On the contrary, low mobilities and thermally activated hopping transport are observed for electrons in solid and liquid $\mathrm{He}^{4}$ and in liquid Ne. The conduction electron repels surrounding rare-gas atoms, creating a lattice expansion or a bubble around the electron. This is a well-known example of self-trapping, in which electrons become stabilized by localizing themselves in the potential well as a result of the self-induced lattice distortion. The composite particle, i.e. localized electron plus strong lattice distortion, is called a small polaron and is discussed further below. The occurrence of a bubble is associated with the negative electron affinity in condensed 
phases of the light rare gases. For these atoms, the short-range pseudopotential is dominated by the strongly repulsive interaction between the excess electron and the atomic core. In heavier rare-gas atoms and molecules the polarizability becomes significant, softening the sharp Pauli repulsion by the core. The interaction between an excess electron and a rare-gas lattice becomes attractive for $\mathrm{Kr}$ and $\mathrm{Xe}$.

In angle-resolved TPPE study of various alkanes on the $\mathrm{Ag}(111)$ surface, both localized and delocalized states were found in the spectra taken with a laser pulse duration of six picoseconds [81]. For n-alkanes and cyclohexane, in addition to the dispersive feature normally expected for the delocalized electrons $\left(m^{*} \approx 1.2 m_{\mathrm{e}}\right)$, a nondispersive peak was observed and attributed to localized electron states. However, layers of neopentane do not induce a localized state even up to trilayer thicknesses. Based on the non-time-resolved TPPE data, it was proposed that the geometry of adlayer molecules and their resultant low-energy electron scattering properties [125] are linked to the electron localization observed in the 2-D films [81]. The results were correlated with excess electron mobility data for nonpolar liquids, since mobility in liquid neopentane is two to three orders of magnitude higher than in n-alkanes or cyclohexane [106]. Localization was not seen for the $n=2$ electron in the presence of monolayers and bilayers of some straight chain alkanes, presumably because the electron density is further away from the interface and thus the interaction with the adlayer is less. This was the first work to observe localization of excess electrons at an interface as a nondispersive feature in angle-resolved photoemission. 


\subsection{Results of Femtosecond Time-Resolved Study}

In order to elucidate the time scale of the localization process and the mechanism, we performed a femtosecond time-resolved study for one to three layers of n-heptane on $\operatorname{Ag}(111)[66,126]$. The pump and probe wavelengths used in the experiments were $300 \mathrm{~nm}$ and $600 \mathrm{~nm}$, respectively. Fig. 6.1 shows the surface projected $\mathrm{Ag}(111)$ valence and conduction bands and $n=1$ image potential states upon adsorption of a bilayer of $\mathrm{n}$-heptane. A wide range of delocalized states ( $k_{\|}$from 0 to $0.4 \AA^{-1}$ ) can be populated by $300 \mathrm{~nm}$ pump pulses.

In n-heptane bilayer data taken at $120 \mathrm{~K}$ with two different pump/probe delays, a dispersive feature with $m^{*}=1.2 m_{\mathrm{e}}$ appears at zero time delay (Fig. 6.2A), whereas a non-dispersive feature appears in the spectra taken at 1670 fs delay (Fig. 6.2B). These two features correspond to the delocalized and localized states which appear to coexist in the previously published picosecond TPPE data [81]. The femtosecond TPPE data clearly reveal a delay in the formation of the localized state. Similar behavior is found for a monolayer and a trilayer.

Figure 6.3 shows the dynamics of the localized and delocalized features at $120 \mathrm{~K}$ for various angles. At high angles where the two features are separated, the two dynamic traces were taken from the average of the data points near the corresponding peak maxima in the photoelectron kinetic energy spectra. At low angles where these two features are significantly overlapped, the dynamic traces for the delocalized and localized features are obtained, respectively, from data points located at the higher 


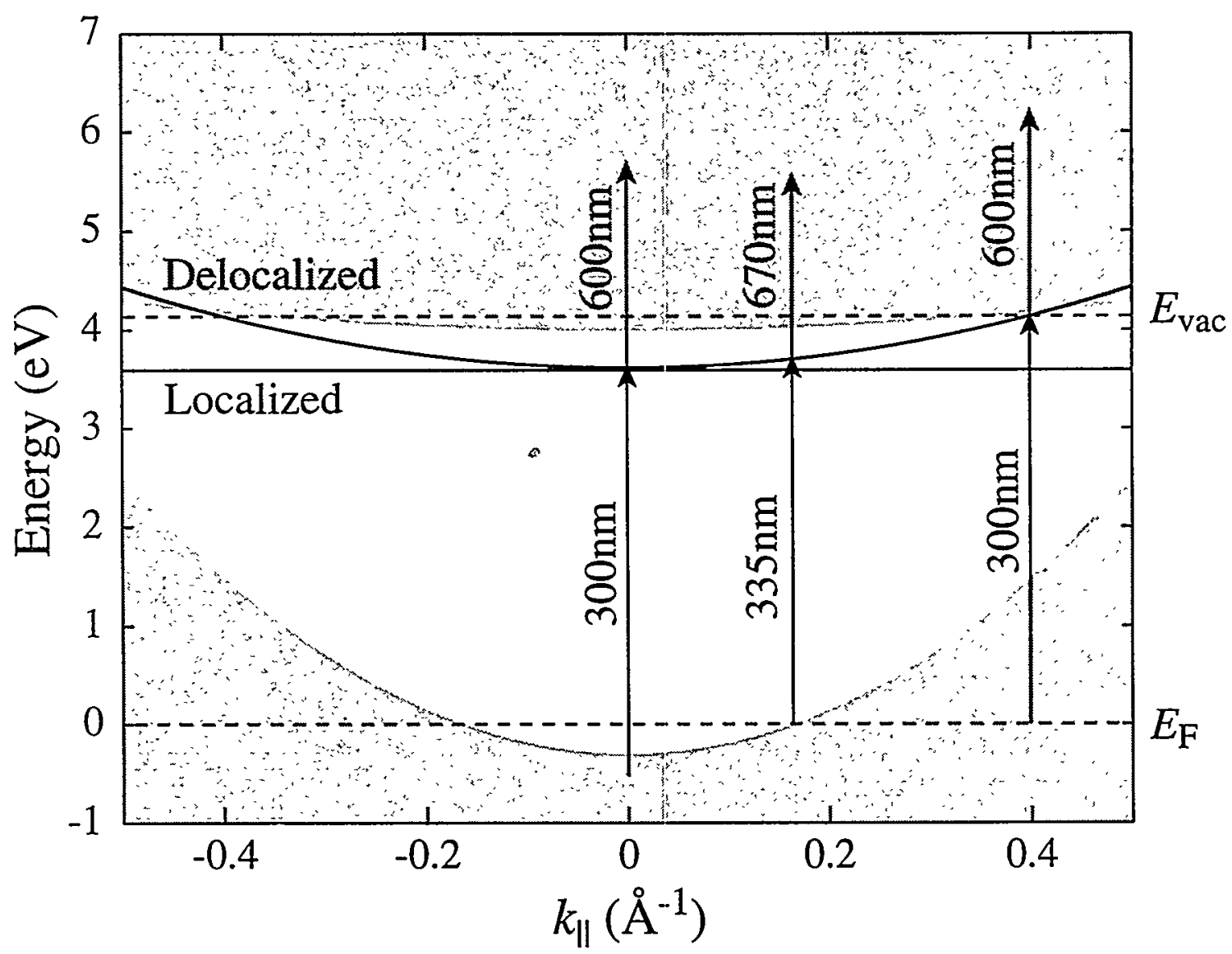

Figure 6.1: The TPPE process is illustrated for bilayer n-heptane/ $\mathrm{Ag}(111)$. Solid lines are the $n=1$ delocalized image potential state and the localized state. The shaded region corresponds to the $\mathrm{Ag}(111)$ bulk bands. $E_{\text {vac }}$ vacuum energy; $E_{\mathrm{F}}$ Fermi Energy. 


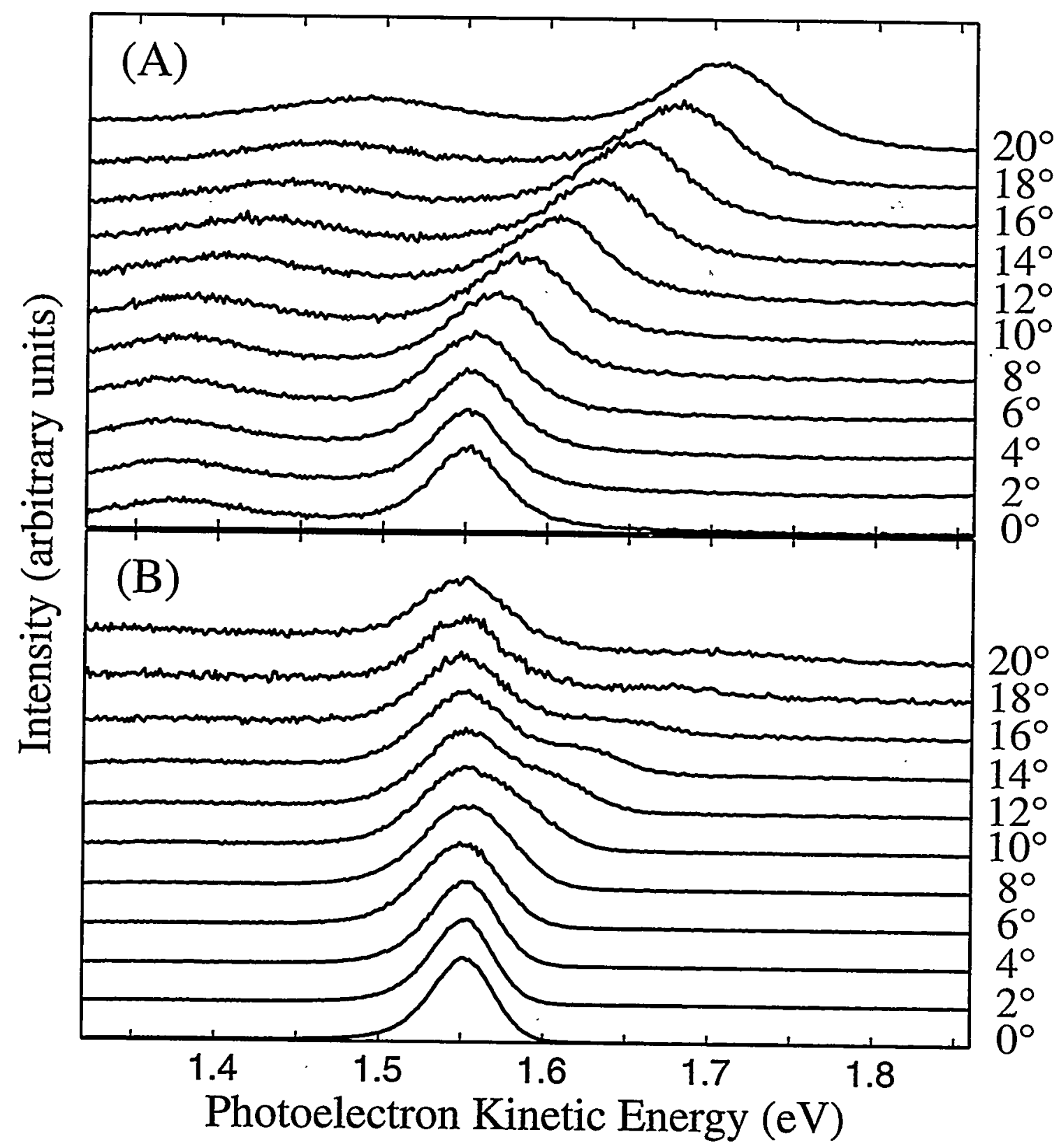

Figure 6.2: Femtosecond angle-resolved TPPE spectra for bilayer n-heptane/Ag(111) taken at $120 \mathrm{~K}$ and a pump-probe delay time of $0 \mathrm{fs}(\mathrm{A})$ and $1670 \mathrm{fs}(\mathrm{B})$. Initially the electron is in the delocalized state with $m^{*}=1.2 m_{\mathrm{e}}$. The electron then becomes localized within a few hundred femtoseconds. The smaller dispersive feature in (B) is the result of small patches of monolayer interspersed with bilayer. 


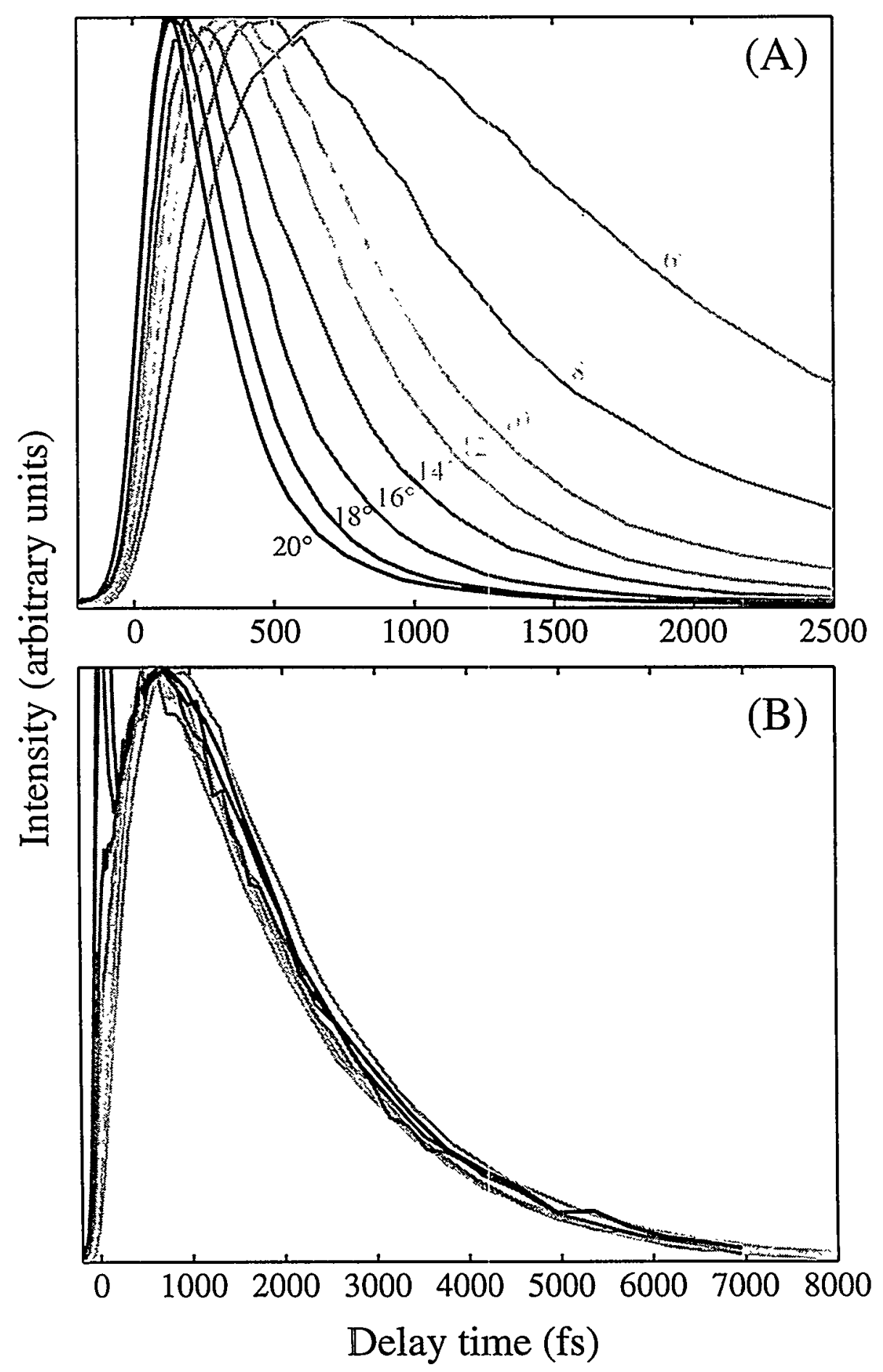

Figure 6.3: Ultrafast time-resolved TPPE traces for (A) delocalized and (B) localized state for bilayer $\mathrm{n}$-heptane on $\mathrm{Ag}(111)$ at various angles and $120 \mathrm{~K}$. The spikes near time-zero in (B) for data at high angles corne from short-lived electrons on small patches of monolayer. 
energy side and the lower energy side of the peak maximum. Clearly, The delocalized feature (Fig. 6.3A) decays more quickly than the localized feature (Fig. 6.3B).

To extract the time constants, we have assumed a model comprising a superposition of Eqn. 4.1 because features are overlapping:

$$
\begin{aligned}
& M_{\mathrm{del}}(t)=A N\left(\tau_{1}^{\mathrm{del}}, \tau_{2}^{\mathrm{del}} ; t\right)+B N\left(\tau_{1}^{\mathrm{loc}}, \tau_{2}^{\mathrm{loc}} ; t\right) \\
& M_{\mathrm{loc}}(t)=C N\left(\tau_{1}^{\mathrm{del}}, \tau_{2}^{\mathrm{del}} ; t\right)+D N\left(\tau_{1}^{\mathrm{loc}}, \tau_{2}^{\mathrm{loc}} ; t\right)
\end{aligned}
$$

Here $M_{\text {del }}(t)$ and $M_{\text {loc }}(t)$ are the dynamic traces for the delocalized and localized features, respectively, provided the instrument function is a delta function. The rise time $\tau_{1}$ and decay time $\tau_{2}$ with the superscripts ${ }^{\text {del }}$ and ${ }^{\text {loc }}$ denote the true time constants for the delocalized and localized states. $A, B, C, D$ are the amplitude parameters. In Fig. 6.2B a smaller dispersive feature at low kinetic energy appears due to the presence of small patches of monolayer interspersed with bilayer. This gives rise to the spikes near time-zero in Fig. 6.3B for data at high angles because electrons on small patches of monolayer are short-lived. It is included in the model for the data as a feature of known time constants (by fitting the monolayer feature separately), adding an additional amplitude parameter to the fit.

Thus the dynamics traces of Fig. 6.3 have been fit by convolving the instrument function with the above model. In order to find the global optimum, random sampling of the initial guess parameter space was employed in the fitting procedure. Within 
the $95 \%$ confidence limit, the extracted rise and decay times shows that the localized feature exhibits the same $360 \pm 140$ fs rise and $1600 \pm 200$ fs decay over the range of angles studied. In contrast, the dynamics of the delocalized states have a strong angle dependence. The rise times vary from $780 \pm 390\left(k_{\|}=0\right)$ to $70 \pm 30$ fs $\left(k_{\|}=0.23 \AA^{-1}\right)$, and the decay times range from $810 \pm 210$ to $200 \pm 50 \mathrm{fs}$, respectively.

The temperature dependence of the dynamics was investigated for a bilayer (Fig. 6.4). As the temperature is lowered from 120 to $50 \mathrm{~K}$, the decay times of the localized and delocalized states exhibit opposite trends in temperature dependence, increasing to $2500 \pm 750 \mathrm{fs}$ for the localized state and decreasing slightly for the delocalized state (e.g. from $270 \pm 50$ to $190 \pm 50$ fs for $k_{\|}=0.19 \AA^{-1}$ ). The rise time decreases from 360 to $200 \pm 140$ fs for the localized state but does not change in the case of the delocalized state.

\subsection{Survey of Possible Mechanisms}

A spatially localized state can be considered as a superposition of many $k_{\|}$plane waves. These localized electrons exhibit no angle dependence in their dynamics (Fig. 6.3B), showing that all Fourier components of the localized state have the same dynamics, as expected for photoemission from a single state. The lifetime of the localized state is attributed to the direct decay back into the metal. Previous chapter demonstrated an exponential increase of image electron lifetime with layer thickness due to tunneling back into the metal [45]. That result explains the localized state 


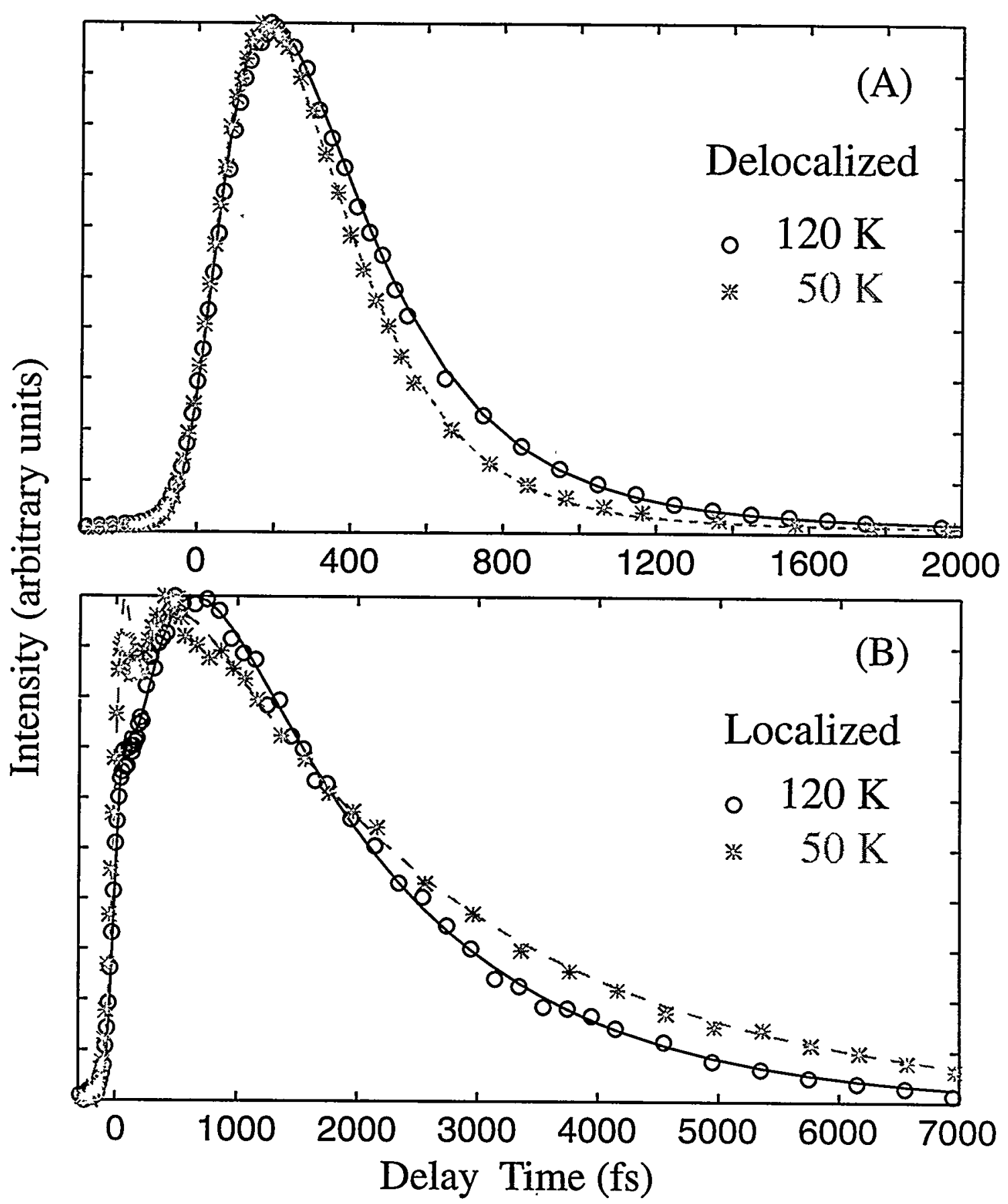

Figure 6.4: Temperature dependence of the dynamics of the (A) delocalized and (B) localized state for bilayer $\mathrm{n}$-heptane $/ \mathrm{Ag}(111)$ at $16^{\circ}$. Data were taken at $120 \mathrm{~K}(\mathrm{\circ})$ and $50 \mathrm{~K}\left({ }^{*}\right)$. The best fits obtained by iterative reconvolution are represented by solid lines for $120 \mathrm{~K}$ and dashed lines for $50 \mathrm{~K}$. 
decay mechanism. While the localized state shows no angle dependence in its lifetime, it does show temperature dependence. As the temperature is lowered, the lifetime of the localized state becomes longer. This temperature dependence is attributed primarily to the fact that the localized state becomes more bound at lower temperature (binding energy varying from $-0.52 \mathrm{eV}$ at $120 \mathrm{~K}$ to $-0.54 \mathrm{eV}$ at $50 \mathrm{~K}$ ) and thus experiences a higher potential barrier for tunneling.

The strong $k_{\|}$-dependence of delocalized state lifetime, however, suggests that decay mechanisms other than direct decay back into the metal are operative. One possibility is intraband momentum relaxation to a different $k_{\|}$state and such relaxation may in turn result in a angle dependence of the rise time of the delocalized state. Our data show a much weaker $k_{\|}$-dependence than the $k_{\|}^{4}$-dependence of linewidth broadening predicted for the Auger process on bare metal surfaces $[33,41]$. We can therefore eliminate the contribution from this process because the adlayer will weaken interaction between the image electrons and the bulk metal electrons. Another possibility is the scattering of the image electrons with phonons of the alkane layers. However, the temperature dependence study on bilayer for a range of angles (Fig. 6.4 on the preceding page and Fig. 6.5 on page 104) shows that delocalized states at $50 \mathrm{~K}$ decay faster than at $120 \mathrm{~K}$, contradicting the phonon scattering theory prediction and indicating the need for other mechanisms. We note that the decay times of the delocalized states are of the same order as the rise time of the localized state. In fact, they correspond even at different temperatures. Both rates become faster as the tem- 
perature is lowered from 120 to $50 \mathrm{~K}$. Such a temperature dependence of the $n=1$ delocalized state is opposite to that of the bilayer $n=2$ state (Fig. $6.6^{1}$ on page 105) and bare $n=1$ state (Fig. 4.11 on page 63 ) which are delocalized and do not show localization. These observations indicate that the delocalized electron primarily decays into the localized state and the localization time not only depends on its initial parallel momentum but also exhibits a non-Arrhenius temperature dependence.

- In the search for a plausible localization mechanism, we can eliminate some of the possible candidates based on the following experimental observation. First, the previous study of various alkane $/ \mathrm{Ag}(111)$ interfaces [81] extensively investigated the effects of different layer preparation methods and annealing procedures on localization and found no difference in the relative intensity of the localized peak [129]. Second, the proportion of monolayer patches interspersed with bilayer (apparent in Fig. 6.2B) can be varied by a factor of 5 without variation in the bilayer dynamics. These experiments shows that no amounts of defects or disorder in the layer that we could control had any significant effects on localization. Third, the localized peak and the delocalized peak are very close in energy at $k_{\|}=0$ with a difference $\Delta E_{0} \leq 10 \mathrm{meV}$ (Fig. 6.2). The fact that $\Delta E_{0}$ is small suggests that impurity-enhanced localization is unlikely because one would expect $\Delta E_{0}$ to be much larger than $10 \mathrm{meV}$ if localization

\footnotetext{
${ }^{1}$ Interestingly, the inverse lifetime of the $n=2$ state for a bilayer n-heptane exhibits a nearly linear dependence on the electron energy parallel to the interface, $E_{\|}$. This $k_{\|}^{2}$ dependence, instead of $k_{\|}^{4}$, suggests that the Auger scattering with the bulk metal electrons is weak in this system, consistent with the repulsive electron affinity of the alkane layer. Recently, a time- and angle-resolved TPPE study of the $n=1$ state for a monolayer and bilayer of $\mathrm{Xe}$ on $\mathrm{Ag}(111)$ shows that the inverse lifetime depends linearly on $k_{\|}[127,128]$. These results are not as yet well understood and further investigation is underway.
} 


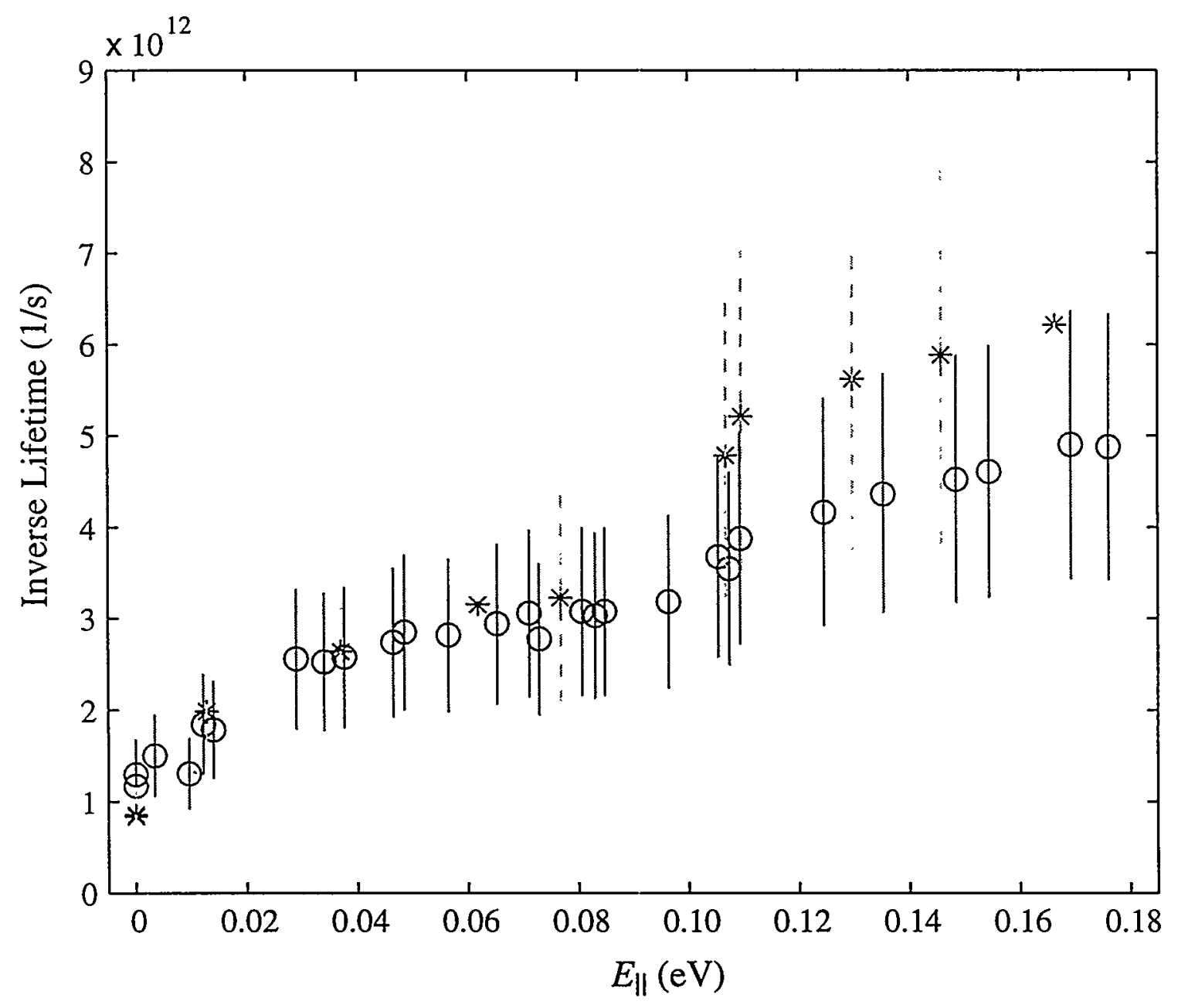

Figure 6.5: Temperature dependence of the dynamics of the delocalized state for bilayer $\mathrm{n}$-heptane $/ \mathrm{Ag}(111)$ at various angles. The inverse lifetime is plotted as a function of the electron energy parallel to the interface, $E_{\|}=\hbar^{2} k_{\|}^{2} / 2 m^{*}$. Data were taken at $120 \mathrm{~K}(\mathrm{o})$ and $50 \mathrm{~K}\left({ }^{*}\right)$. The $95 \%$ confidence limit for each data point is indicated by the vertical line. 


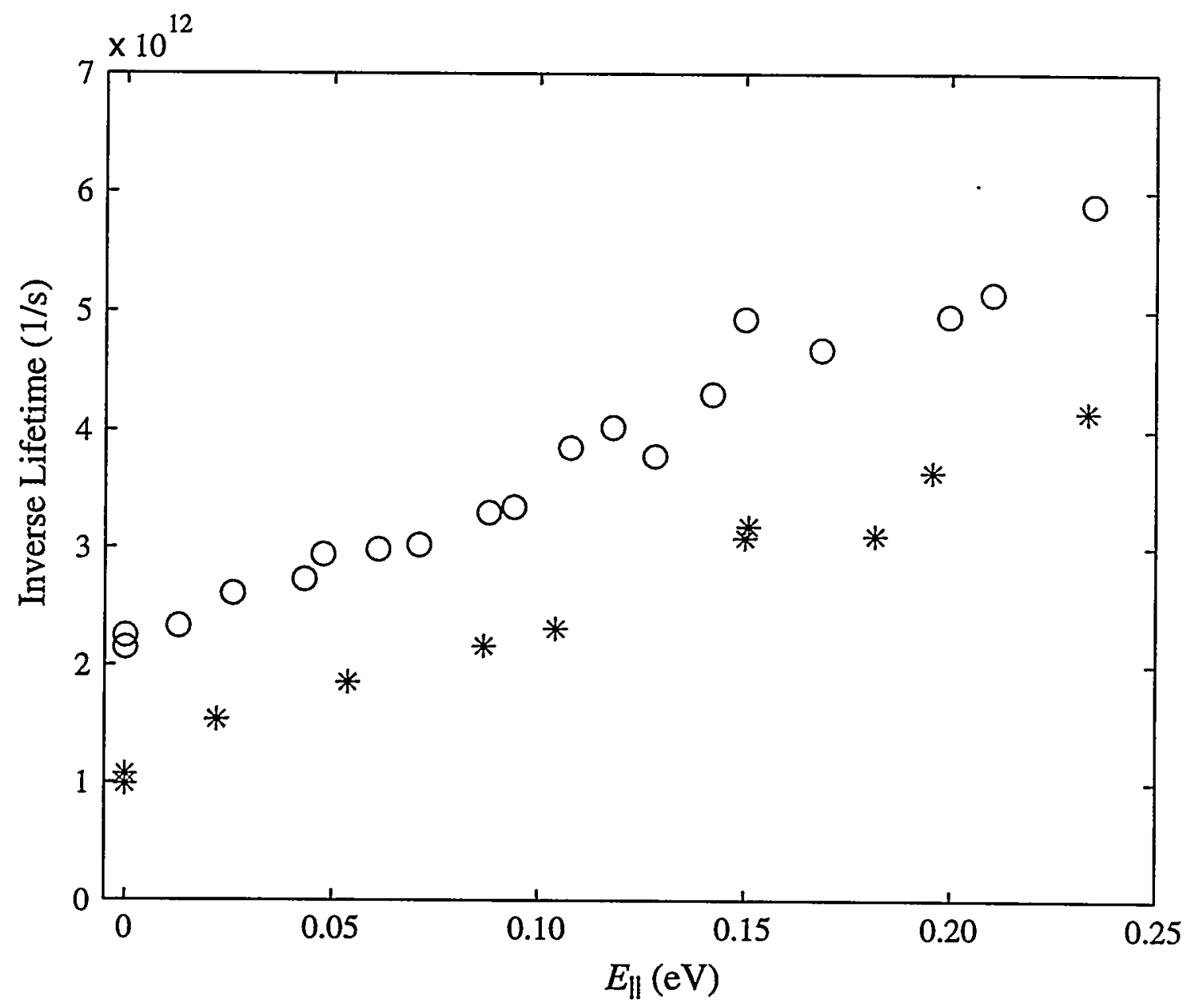

Figure 6.6: Temperature dependence of the dynamics of the $n=2$ image state for bilayer $\mathrm{n}$-heptane $/ \mathrm{Ag}(111)$ at various angles. The inverse lifetime is plotted as a function of the electron energy parallel to the interface, $E_{\|}=\hbar^{2} k_{\|}^{2} / 2 m^{*}$. Data were taken at $120 \mathrm{~K}(\circ)$ and $50 \mathrm{~K}\left({ }^{*}\right)$. 
were caused by electron trapping around impurities chemically distinct from n-alkane. Furthermore, it appears that the energy of the localized state is pinned to the bottom of the delocalized band regardless of layer composition (chain length) and thickness. For example, the $n=1$ binding energy shift upon adding a third layer is $\approx 80 \mathrm{meV}$ for n-pentane. Yet the localized state peak associated with the bilayer also moves $\approx 80 \mathrm{meV}$, so that it is still $\leq 10 \mathrm{meV}$ below the bottom of the band. The invariance of $\Delta E_{0}$ versus layer thickness rules out localization due to confinement on small adsorbate islands [130] or vacancies. On a monolayer the depth of the potential well formed by vacancies of one-monolayer thickness is higher than that on a bilayer. The confinement energy shift on the former has to be higher by the same amount in order to make $\Delta E_{0}$ to be the same. One would not expect this to be a general case but at the mercy of a particular layer growth condition. These observations indicate that the localization phenomenon and dynamics are not controlled by defects in the layer, but rather are linked to the intrinsic properties of the layer. As shown below, these data are well explained by a 2D small polaron model for localization, and the $k_{\|^{-}}$ dependence of the delocalized states decay times is related to the lattice relaxation dynamics which lead to self-trapping.

\subsection{Small Polaron Formation}

The self-trapping of electrons in small polarons, originally conceived by Landau [131], is conceptually simple. Electrons in rigid periodic lattices are delocalized, 
forming Bloch bands that are free-electron-like. In a deformable lattice, however, the electron-lattice interaction causes an electron to always carry with it a self-induced lattice deformation. The composite particle is called a polaron. "Small" polarons involve strong lattice distortion and the wavefunctions of the electrons are self-trapped to dimensions of a lattice spacing. Their motion between lattice sites can be described as activated hopping [132], very different from band-like behavior. These self-trapped carriers and the associated lattice relaxation occur in many nonmetallic systems $[133$, 124], and greatly affect a wide range of phenomena, such as photochemical defect formation [134], atomic desorption from solid surfaces [135], and various properties of high $T_{c}$ superconducting oxides $[136,137]$.

An electron in a crystal is subject to a competition between delocalization and localization tendencies. The degree of localization of electrons is determined by such a competition. Delocalization is favored because an electron residing at the bottom of a band has low kinetic energy. (Fig. 6.7, point F). In order to localize the electron at a single site without lattice distortion, energy is required to construct an electron wavepacket from all Bloch waves in the band (Fig. 6.7, point C). The energy expended, termed the localization energy $E_{\text {loc }}$, can be estimated from the half width of the band, $B$ [138]. On the other hand, localization through small polaron formation is encouraged because the electron can create for itself an attractive potential well by polarizing and displacing the atoms surrounding it (Fig. 6.7, point S), thus releasing the lattice relaxation energy $E_{\text {rel }}$. Qualitatively speaking, the localized or self-trapped 


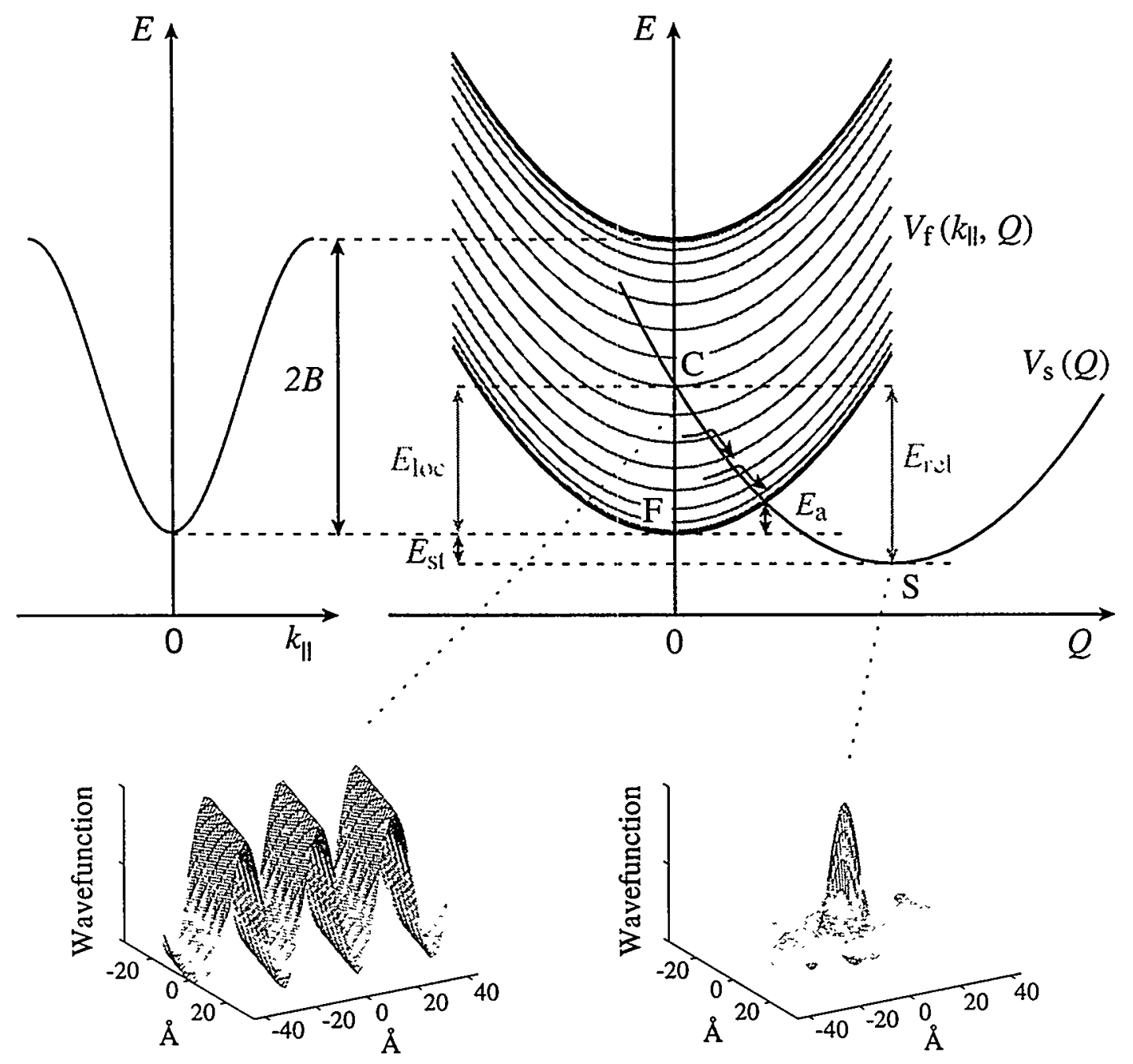

Figure 6.7: Configuration coordinate diagram for self-trapping of an electron. Curves $V_{\mathrm{f}}\left(k_{\|}, Q\right)$ and $V_{\mathrm{s}}(Q)$ are associated, respectively, with the delocalized states and the self-trapped state. $Q$ is the phonon coordinate responsible for self-trapping. Each curve in the $V_{\mathrm{f}}$ manifold represents a different $k_{\|}$state with a band energy $E_{\|}\left(E_{\|}=0\right.$ at $\left.k_{\|}=0\right)$. The width of the band $(2 B)$, localization energy $\left(E_{\text {loc }}\right)$, lattice relaxation energy $\left(E_{\text {rel }}\right)$, self-trapping energy $\left(E_{\text {st }}\right)$ and the activation energy of self-trapping $\left(E_{\mathrm{a}}\right)$ for $k_{\|}=0$ are depicted. Arrows indicate the classical barrier crossing from the delocalized to the localized state. Schematic illustration of the electron wavefunction at n-heptane $/ \mathrm{Ag}(111)$ interface is also shown. Bottom left: an electron in a delocalized state with $k_{\|}=0.22 \AA^{-1}$. Bottom right: an electron self-trapped in a localized state which is assumed to be a 2-D wavepacket composed of all $k_{\|}$in the first surface Brillouin zone of the n-heptane lattice. The spatial extent of the resulting wavefunction is comparable to the unit mesh dimensions. 
state of the electron will be energetically more favorable and the small polaron will form when the self-trapping energy is positive:

$$
E_{\mathrm{st}}=E_{\mathrm{rel}}-E_{\mathrm{loc}}>0 .
$$

The balance between these opposing tendencies depends on the dimensionality of the lattice and the nature and the strength of its coupling with the electron. Theoretical analysis has shown $[139,140,141,142]$ that if the length scale of the electron wavefunction is compressed or expanded by a factor $L$ in a system of dimensionality $d$, the kinetic energy of the electron always scales as $L^{-2}$. For a short-range electron-lattice coupling, which is the case for electrons in non-polar alkane solids, the lattice relaxation energy scales as $L^{-d}$. Thus localization is energetically favored over delocalization in $1-D$, and vice versa in $3-D$. In a $2-D$ system, however, these two tendencies scale in the same way with $L$ and are nearly canceled. Thus two dimensionality is often called the marginal case. $[140,141,142,143]$

Our data are well explained by the above 2D small polaron formation model. The time delay between populating the image state and the formation of the localized electrons is a manifestation of the dynamic aspect of small polaron formation. The initial creation of delocalized electrons on the surface (Fig. 6.2A) can be understood as a consequence of the Franck-Condon principle, i.e. optical excitation primarily promotes electrons from delocalized metal states into delocalized image potential 
states without lattice distortion. The excited electrons can then be stabilized by selftrapping and become localized at a later time (Fig. 6.2B) because the lattice and the molecules that comprise it respond to the presence of an excess electron on the time scale of the lattice and molecular vibrational periods.

The small self-trapping energy, as measured by $\Delta E_{0} \leq 10 \mathrm{meV}$, indicates the existence of a delicate balance between the delocalizing and localizing tendencies in this system. This is consistent with a $2-D$ self-trapping picture based on the invariance of $\Delta E_{0}$ versus layer thickness. Each additional alkane layer only modifies the interfacial potential normal to the interface, without affecting the trapping process parallel to the interface, leaving $E_{\text {st }}$ unchanged. The self-trapped electron wavefunction can still be decomposed into a lateral component which is localized to a dimensions of an alkane unit surface mesh, and a vertical component which is the usual 1-D image state wavefunction. The lifetime of the self-trapped electron is $k_{\| 1}$-independent (Fig. 6.3B on page 98), but is dramatically lengthened with increasing alkane layer thickness as shown in Table 5.1 on page 74 . The data listed in the $n=1$ row actually refer to the decay time of the localized state. The tunneling model in the previous section explains the decay mechanism of the localized state and suggests that the selftrapped electron is localized at the layer-vacuum junction (Fig. 5.6 on page 82) rather than at the layer-metal junction. The self-trapping process is associated with interactions between the electron and the topmost plane of the alkane molecules without rearrangement of the layer below. 
Furthermore, the small polaron model predicts a self-trapping time dependent on the electron's momentum. Inspecting the potential surfaces of the free and selftrapped states (Fig. 6.7 on page 108), one sees that the system has to move from $V_{\mathrm{f}}$ to $V_{\mathrm{s}}$ in order for self-trapping to occur. An energetically favorable pathway to get from $V_{\mathrm{f}}$ to $V_{\mathrm{s}}$ without first reaching the point $\mathrm{C}$ involves thermal activation of the lattice to a configuration where the energy of the system is the same on both sides, i.e. the crossing point of $V_{\mathrm{f}}$ and $V_{\mathrm{s}}$. The energy difference between the crossing point and $V_{f}\left(k_{\|}\right)$at $Q=0$ represents the potential barrier or the activation energy for self-trapping. The self-trapping barrier is large at $k_{\|}=0$, decreases with $k_{\|}$until reaching point $\mathrm{C}$ where there is no barrier, then turns around to increase with $k_{\|}$. Therefore the self-trapping rate will have a strong dependence on $k_{\|}$. On the other hand, the rate at which the self-trapped electrons form will be an averaged sum over self-trapping rates from all $k_{\|}$values [66].

In fact this picture is supported by the wavelength dependence of the rise time of the localized state. With $300 \mathrm{~nm}$ pump/ 600nm probe, a wide range of $k_{\|}$states can be populated (Fig. 6.1 on page 96), and they can all decay into the localized state giving rise to a $360 \mathrm{fs}$ rise. If a narrower range of high $k_{\|}$states can be selectively populated, the rise time should become shorter since only states with high $k_{\|}$and fast self-trapping rate decay into the localized state. This prediction is born out by the results obtained for $335 \mathrm{~nm}$ pump/670 nm probe by which only states with $k_{\|} \sim 0.15-0.17 \AA^{-1}$ can be populated, resulting in a faster rise by a factor of $\sim 2$. 
The above description of the self-trapping process by the crossing of two potential energy levels spanned by a generalized nucleas: coordinate is analogous to the treatment found in the theory for small polaron hopping [132] as well as the theory for electron transfer reactions [144]. In order to quantitatively model the self-trapping dynamics, we employed a recently developed electron transfer theory [145] based on path integral approach. An explanation of the theory is given in the next section.

\subsection{Electron Transfer Theory}

Electron transfer is one of the most fundamental and important processes in physical, chemical, and biological systems $[146,144]$. Rates of electron transfer in various systems span some twenty orders of magnitude. Experimental and theoretical research on this subject has been actively pursued for many decades, and both classical and quantum-mechanical interpretations have been proposed $[147,148,149,150,151]$. Traditional theoretical treatments have focused on electron transfer between weakly coupled electron transfer sites. In this limit, lowest order perturbation theory in the electronic coupling can be used, yielding the well-known golden rule results. Strongly interacting systems, however, are much less studied and an exact rate expression is not yet available. Furthermore, a general rigorous theory unifying two extreme cases has not been developed until recently and one often resorts to semiclassical treatments, such as making use of the Landau-Zener formula for the transition probability $[152,153,154]$ 
This section is devoted to explain and review the recent development of the spinboson Hamiltonian model of electron transfer by Stuchebrukhov and Song $[155,145]$. This model Hamiltonian in conjunction with path-integral techniques provides excellent tools for studying the strong coupling limit and for discussing a unified approach to adiabatic and nonadiabatic regimes. The path-integral approach $[156,157,158]$ is more convenient than the standard wavefunction formulation because it allows the inclusion of the coupling to the heat bath as an influence functional and because it makes the transition to the classical limit much easier. An excellent text on path integral and quantum theory of liquids has been written by Chandler [159].

After reviewing the theory, calculations under a few limiting cases is performed to test the validity of their theory and to demonstrate the effects of coupling strength, and the role of quantum modes.

\subsubsection{Spin-Boson Hamiltonian}

In electron transfer reactions the reactant and product electronic states can usually be approximated as a two electronic-state system: If the solvent and the nuclear motion of the the reactants and products are described as a harmonic bath, the electron transfer can be viewed as an electron jump between the two states modulated by a harmonic bath. This kind of system can be formally described by the spin-boson 
Hamiltonian $[160,161,159]$

$$
\begin{aligned}
& \mathcal{H}=\mathcal{H}_{\mathrm{TLS}}+\mathcal{H}_{\mathrm{SB}}+\mathcal{H}_{\mathrm{B}} \\
& =H_{\mathrm{if}} \sigma_{x}+\frac{\Delta \mathcal{E}}{2} \sigma_{z}+\sigma_{z} \sum_{i} c_{i} y_{i}+\sum_{i}\left(\frac{p_{i}^{2}}{2 m_{i}}+\frac{1}{2} m_{i} \omega_{i}^{2} y_{i}^{2}\right) \text {. } \\
& \sigma_{x}=\left[\begin{array}{ll}
0 & 1 \\
1 & 0
\end{array}\right] \quad \sigma_{z}=\left[\begin{array}{cc}
1 & 0 \\
0 & -1
\end{array}\right]
\end{aligned}
$$

Here $\mathcal{H}_{\mathrm{TLS}}, \mathcal{H}_{\mathrm{SB}}$, and $\mathcal{H}_{\mathrm{B}}$ are the Hamiltonian for the two-level system (TLS), spinboson coupling Hamiltonian, and bath Hamiltonian, respectively, $H_{\text {if }}$ is the coupling matrix element associated with resonance between unsolvated electron transfer species in the initial and final states, $\Delta \mathcal{E}$ is the thermodynamic driving force or exothermicity of the reaction, $\sigma_{x}$ and $\sigma_{z}$ are Pauli matrices in the two-state (reactant $\left.\mid+\right)$ and product $|-\rangle)$ representation, $y_{i}, p_{i}, m_{i}$, and $\omega_{i}$ are, respectively, the coordinate, momentum, mass and frequency of the $i$ th harmonic oscillator representing the bath. The term 'spin' is used since the two electronic states are taken as two states of a pseudospin variable, and 'boson' comes from that the harmonic bath follow the BoseEinstein statistics. In the absence of the transferring species, all modes of the bath are symmetric with no net polarization or displacements. The spin system coupled linearly to the linear combination of the bath modes, $q=\sum_{i} c_{i} y_{i}$.

As a function of $q$, the free energy surfaces in the nonadiabatic and adiabatic limits are shown in Fig. 6.8 on the facing page. If nuclei move very slowly such that the nuclear dynamics can be neglected, there is plenty of time spent in the curve- 
(A) Nonadiabatic

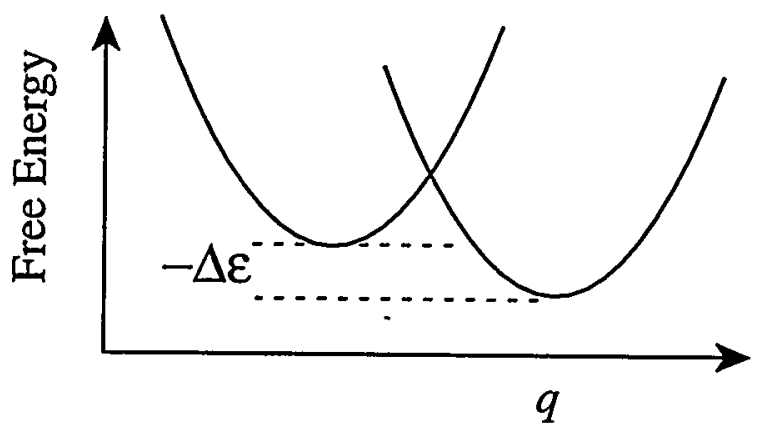

(B) Adiabatic

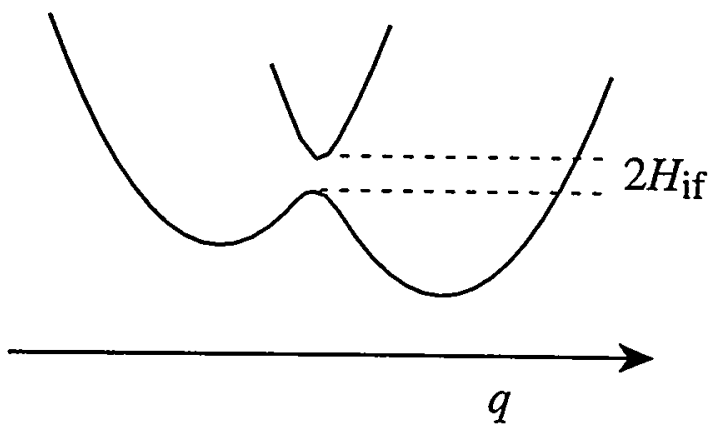

Figure 6.8: Free energy surfaces for the (A) nonadiabatic and (B) adiabatic limits.

crossing region for electron transfer. The reaction happens with high transmission coefficient. This is the adiabatic limit which corresponds to electronic coupling being strong enough to ensure that the system follows the lower pathway at all times. The opposite limit then is the nonadiabatic limit corresponding to weak electronic coupling. The limits for the nonadiabatic and adiabatic cases can be estimated by $H_{\text {if }} \ll k_{\mathrm{B}} T$ and $H_{\text {if }} \gg k_{\mathrm{B}} T$, respectively. If the bath modes can be treated classically, a valid approach in the high temperature limit, the electron transfer rate is given by $[146,144,132]$

$$
\begin{gathered}
k=A^{\prime} \mathrm{e}^{-\left(\Delta \mathcal{E}+E_{\mathrm{r}}\right)^{2} / 4 E_{\mathrm{r}} k_{\mathrm{B}} T}, \\
A^{\prime}= \begin{cases}\left(\frac{\pi}{\hbar^{2} E_{\mathrm{r}} k_{\mathrm{B}} T}\right)^{1 / 2} H_{\mathrm{if}}^{2} & \text { nonadiabatic, golden-rule } \\
\omega_{0} / 2 \pi & \text { adiabatic, Landau-Zener }\end{cases}
\end{gathered}
$$

where $E_{\mathrm{r}}$ is the reorganization energy and $\omega_{0}$ is the frequency of the harmonic poten- 
tial well.

To understand the development of the unified theory [145] that includes these two limits on equal footing and is able to treat the bath modes quantum-mechanically, the concept of the complex partition function is introduced next.

\subsubsection{Rate Constant and Partition Function}

In formulating a way to calculate rate constant, a relation between the escape rate, $k$, and the imaginary part of the free energy, $F$, for metastable systems is usually used. Consider the left hand side of the lower potential curve in Fig. 6.8B on page 115. It is a system with a localized metastable ground state and a saddle point through which the system can escape to the true ground state. At low temperatures the system is mainly in the low-lying metastable states. These are wavefunctions that vanish at $-\infty$, are standing waves normalized to 1 in the well, and give an exponentially small probability current at positive $q$. The nonconservation of probability current requires that the energy of the metastable level have an (exponentially small) imaginary part, as demonstrated long ago by Langer $[162,163]$. In his discussion, he showed that the partition function of a metastable system can be rigorously defined only as an analytic continuation in the parameter space from the region where the system is stable and well defined to the region where the system is metastable. He demonstrated this idea first with a model potential of the form $a q^{2}+b q^{4}$ (Fig. 6.9). For small positive $b$ this is simply a potential for a harmonic oscillator with a small quartic anharmonicity. The 

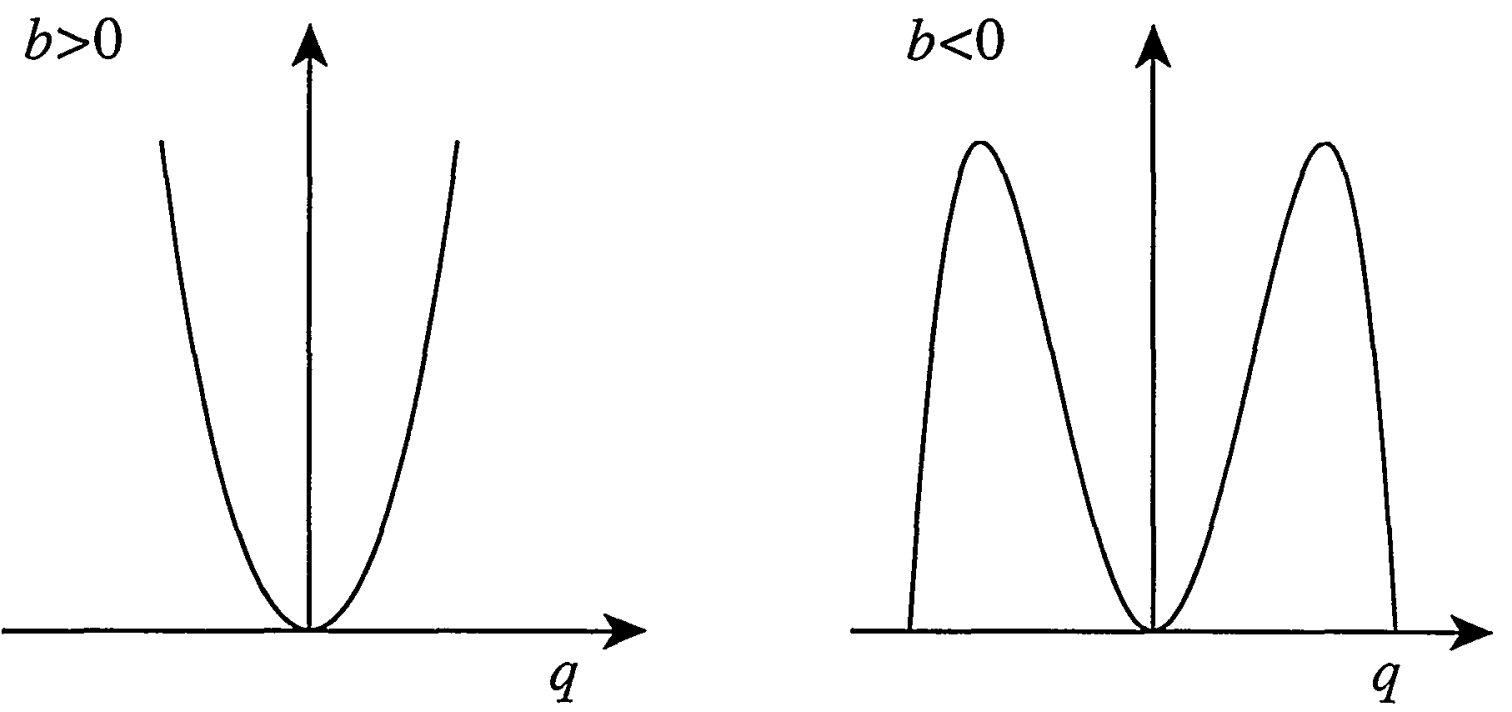

Figure 6.9: A model potential: $a q^{2}+b q^{4}$, where $a>0$.

wavefunction is symmetric and is a pure decaying exponential at infinity. However, if $b$ becomes negative, the potential bends over and becomes negative for large $q$. In this case, no strictly stationary eigenstate exists. The decaying exponential becomes a pure outgoing or incoming wave. This new boundary condition is non-Hermitian and must lead to a complex eigenvalue $\lambda$. The freedom of choice between incoming and outgoing waves at infinity reflects the fact that $b=0$ is a branch point of $\lambda(b)$. The energy of the metastable state $\lambda(b<0)$ is only defined by analytic continuation and its imaginary part is related the decay rate of the state. This concept has been generalized and followed up by many other papers $[164,165]$.

The rate-energy relation can be obtained by a simple argument. Consider the time-dependent wavefunction of the $n$th level, $\left|\psi_{n}(t)\right\rangle=\mathrm{e}^{-\mathrm{i} E_{n} t / \hbar}\left|\psi_{n}(0)\right\rangle$. If the energy $E_{n}$ is regarded as having a real part and a small imaginary part $E_{n}=E_{n}^{0}-\mathrm{i} \hbar \gamma_{n} / 2$, 
then the probability density of finding the system in the $n$th level will decay with a escape rate $\gamma_{n}$, i.e. $\left|\psi_{n}(t)\right|^{2}=\mathrm{e}^{-\gamma_{n} t}\left|\psi_{n}(0)\right|^{2}$. Therefore, the relation between $\gamma_{n}$ and the imaginary part of the energy is $\gamma_{n}=-(2 / \hbar) \operatorname{Im} E_{n}$. Taking a Boltzmann average of $\gamma_{n}$, the average rate of escape from all metastable levels becomes

$$
\begin{aligned}
k & =\sum \gamma_{n} \mathrm{e}^{-\beta E_{n}^{0}} / \sum \mathrm{e}^{-\beta E_{n}^{0}} \\
& =\left(\frac{2}{\beta \hbar}\right)\left(\operatorname{Im} \sum \mathrm{e}^{-\beta\left(E_{n}^{0}-\mathrm{i} \hbar \gamma_{n} / 2\right)} / \sum \mathrm{e}^{-\beta E_{n}^{0}}\right) \\
& =\left(\frac{2}{\beta \hbar}\right) \frac{\operatorname{Im} Z}{Z^{0}} \\
& =-\left(\frac{2}{\hbar}\right) \operatorname{Im} F,
\end{aligned}
$$

where $\beta=1 / k_{\mathrm{B}} T$, and the second equality holds for small $\gamma_{n}$, namely, $\beta \hbar \gamma_{n} \ll 1$. $Z$ denotes the complex partition function containing a real part $Z^{0}$ and a small imaginary part $Z^{\prime}$. The third equality follows from $F=-\beta \ln Z$ and retaining the expansion of natural logarithm to the first order in $Z^{\prime} / Z^{0}$.

Equation 6.8 is accurate for a low temperature regime where only metastable levels far below the barrier contribute to the rate through tunneling. As the temperature increases, the levels lying above or near the barrier top become important. For high enough temperatures, the rate will become dominated by transitions at and above the barrier top. Because of greater density of state there, a classical description is expected to become approximately valid. The temperature $T_{c}$ of the crossover between the quantum and classical regimes is estimated by $k_{\mathrm{B}} T_{c}=\hbar \omega_{b} / 2 \pi[165,166]$, where 
the barrier is approximated by an inverted parabola of frequency $\omega_{b}$. The prefactor in Eqn. 6.8 on the facing page becomes $\omega_{b} / \pi$ for $T>T_{c}$.

Applying to the electron transfer reaction, the forward reaction rate constant has the form $[167,155,145]$

$$
\begin{gathered}
k=A \frac{\operatorname{Im} \int_{-\mathrm{i} \infty}^{+\mathrm{i} \infty} Z(R) d R}{Z_{R}^{0}} \\
A= \begin{cases}1 / \beta \hbar & \text { if } T<T_{c} \\
\omega_{b} / 2 \pi & \text { if } T \geq T_{c}\end{cases}
\end{gathered}
$$

where $Z(R)$ is the partition function of the whole system with fixed centroid of the reaction coordinate, $R$, analytically continued into the complex plane. $Z_{R}^{0}$ is the partition function of the reactant. The quantum centroid coordinate method, introduced by Gillan [166], can be used to effectively sample the infrequent events in a quantum system and hence acquire information on real time dynamics [168]. The main physical idea underlying this method is in separation of statistical and pure dynamical contributions to the reaction rate. In its application to electron transfer reactions it is necessary to treat centroid reaction coordinate as a complex variable $[167,145]$ in order to have a well defined integral over $R$ in Eqn. 6.10. The strategy of calculation is then the following. First, calculate the partition function for a fixed real centroid coordinate, then make analytic continuation, and integrate over the contour running from $-\mathrm{i} \infty$ to $+\mathrm{i} \infty$. The contour can be deformed to pass the saddle point of 
the integrand which gives the largest contribution to the integral and represents the transition state of the reaction.

\subsubsection{Partition Function and Equivalent Ising Model}

The partition function of the whole system is given by

$$
Z=\left\langle+\left|\operatorname{Tr} \mathrm{e}^{-\beta \mathcal{H}}\right|+\right\rangle+\left\langle-\left|\operatorname{Tr} \mathrm{e}^{-\beta \mathcal{H}}\right|-\right\rangle=Z_{+}+Z_{-}
$$

where the trace is taken over all of the bath coordinates. The electronic coupling in Eqn. 6.4 on page 114 is treated as a perturbation and the Hamiltonian is written as $\mathcal{H}=\mathcal{H}_{0}+\mathcal{H}^{\prime}$, where $\mathcal{H}^{\prime}=H_{\mathrm{if}} \sigma_{x}$ and $\mathcal{H}_{0}$ is the rest of the Hamiltonian. That is, in the two-state representation, the Hamiltonian is written as

$$
\mathcal{H}=\left[\begin{array}{cc}
\mathcal{H}_{0+} & 0 \\
0 & \mathcal{H}_{0-}
\end{array}\right]+\left[\begin{array}{cc}
0 & H_{\mathrm{if}} \\
H_{\mathrm{if}} & 0
\end{array}\right], \quad \mathcal{H}_{0 \pm}=\mathcal{H}_{B} \pm\left(\frac{\Delta \mathcal{E}}{2}+\sum_{i} c_{i} y_{i}\right)
$$

and $\mathcal{H}_{B}$ is the harmonic bath Hamiltonian defined in Eqn. 6.4. In the following, the partition function will be expanded as a infinity sum over all perturbation orders. Physically this infinity sum should converge because the partition function should exist. Also, the transmission coefficient will eventually converge to 1 for the adiabatic case. It can not increase forever with the electronic coupling. 
The Boltzmann operator can be expanded as

$$
\mathrm{e}^{-\beta \mathcal{H}}=\mathrm{e}^{-\beta \mathcal{H}_{0}} \sum_{n=0}^{\infty}(-1)^{n} \int_{0}^{\beta} d \tau_{n} \ldots \int_{0}^{\tau_{2}} d \tau_{1} \mathcal{H}^{\prime}\left(\tau_{n}\right) \ldots \mathcal{H}^{\prime}\left(\tau_{1}\right)
$$

where $\mathcal{H}^{\prime}(\tau)=\mathrm{e}^{\tau \mathcal{H}_{0}} \mathcal{H}^{\prime} \mathrm{e}^{-\tau \mathcal{H}_{0}}$. The partition function then reads

$$
\begin{aligned}
& Z_{+}=\left\langle+\left|\operatorname{Tr} \mathrm{e}^{-\beta \mathcal{H}}\right|+\right\rangle \\
& =\operatorname{Tr} \sum_{n=0}^{\infty}\left(H_{\text {if }}\right)^{2 n} \int_{0}^{\beta} d \tau_{2 n} \cdots \int_{0}^{\tau_{2}} d \tau_{1} \mathrm{e}^{-\left(\beta-\tau_{2 n}\right) \mathcal{H}_{0+}} \mathrm{e}^{-\left(\tau_{2 n}-\tau_{2 n-1}\right) \mathcal{H}_{0-}} \ldots \mathrm{e}^{-\left(\tau_{2}-\tau_{1}\right) \mathcal{H}_{0-}} \mathrm{e}^{-\left(\tau_{1}-0\right) \mathcal{H}_{0+}}
\end{aligned}
$$

In this expression, the $n=1$ term corresponds to propagate $|+\rangle$ by $\mathcal{H}_{0+}$ for time $\tau_{1}$, make a transition to $|-\rangle$, propagate by $\mathcal{H}_{0-}$ for the time period $\tau_{2}-\tau_{1}$, make a transition back to $|+\rangle$, then propagate by $\mathcal{H}_{0+}$ for the rest of time $\beta-\tau_{2}$. The higher $n$ terms correspond to doing this $|+\rangle \leftrightarrow|-\rangle$ transition $2 n$ times and one power of the coupling $H_{\text {if }}$ is picked up at each transition. Pictorially, this process is illustrated in Fig. 6.10 on the next page. Due to the trace property, these "kinks" come in pairs.

Therefore, it is convenient to define a $\tau$-dependent Hamiltonian

$$
\begin{gathered}
\mathcal{H}_{0 n}(\tau)=\mathcal{H}_{B}+\frac{\sigma_{n}(\tau)}{2}\left(\frac{\Delta \mathcal{E}}{2}+\sum_{i} c_{i} y_{i}\right) \\
\sigma_{n}(\tau)= \begin{cases}1 & \text { if } \tau \in\left[\tau_{2 i-1}, \tau_{2 i-2}\right), 1 \leq i \leq n+1 \\
-1 & \text { if } \tau \in\left[\tau_{2 i}, \tau_{2 i-1}\right), 1 \leq i \leq n\end{cases}
\end{gathered}
$$


(A) $n=1$

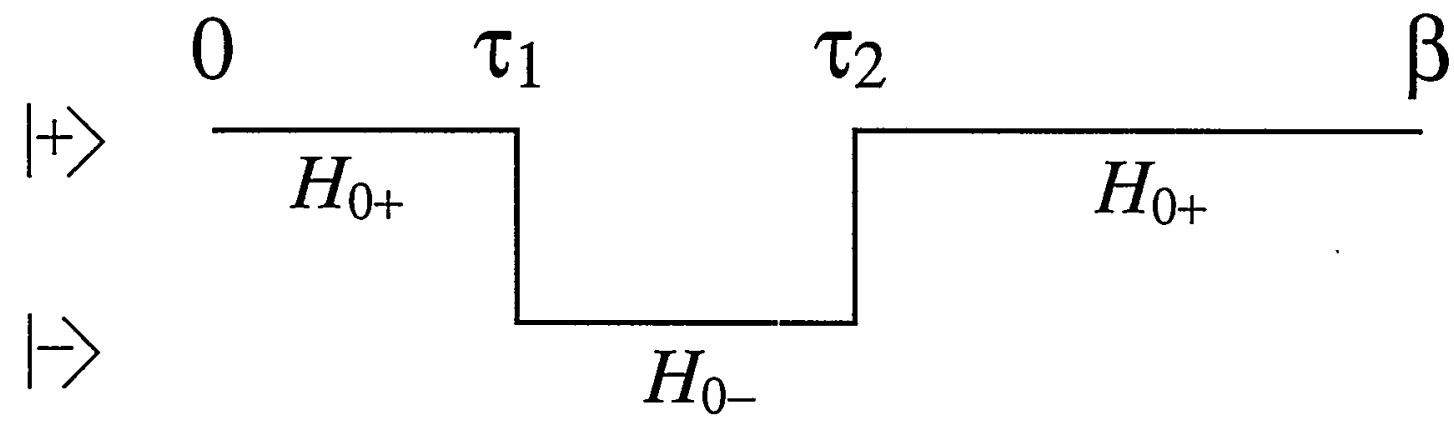

(B) $n=3$

0

$\beta$

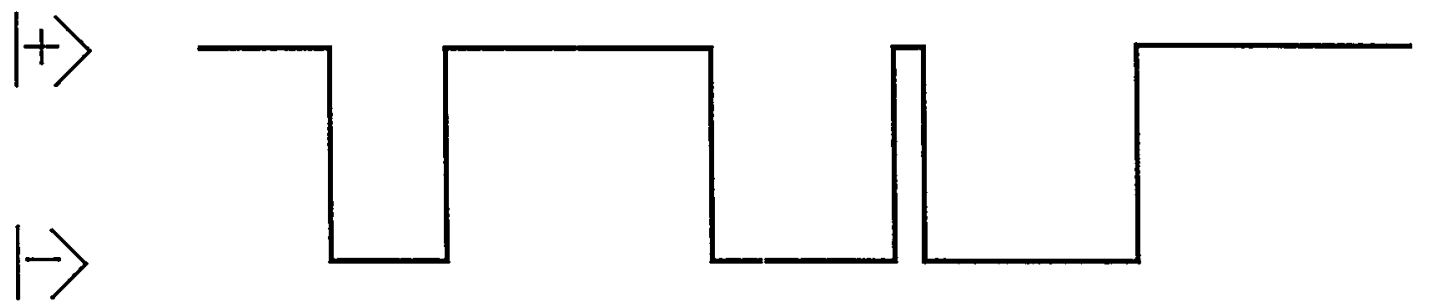

Figure 6.10: Quantum path for the spin $\sigma_{n}(\tau)$ 
where $0 \leq \tau \leq \beta, \tau_{0}=0$, and $\tau_{2 n+1}=\beta$. Thus, $\sigma_{n}(\tau)$ changes sign sequentially $2 n$ times at the points $\tau_{1}, \tau_{2}, \ldots, \tau_{2 n}$ and it obeys a periodic boundary condition, i.e. $\sigma_{n}(0)=\sigma_{n}(\beta)$, due to the trace property. This quantity explicitly describes the quantum paths of the two-state electronic coordinate. The partition function can be written in terms of $\mathcal{H}_{0 n}(\tau)$

$$
\begin{aligned}
Z_{+} & =\operatorname{Tr} \sum_{n=0}^{\infty}\left(H_{\mathrm{if}}\right)^{2 n} \int_{0}^{\beta} d \tau_{2 n} \cdots \int_{0}^{\tau_{2}} d \tau_{1} \exp \left[\sum_{j=1}^{2 n+1}\left(\tau_{j}-\tau_{j-1}\right) \mathcal{H}_{0 n}(\tau)\right] \\
& =\operatorname{Tr} \sum_{n=0}^{\infty}\left(H_{\mathrm{if}}\right)^{2 n} \int_{0}^{\beta} d \tau_{2 n} \cdots \int_{0}^{\tau_{2}} d \tau_{1} \exp \left[\int_{0}^{\beta} \mathcal{H}_{0 n}(\tau) d \tau\right]
\end{aligned}
$$

A similar procedure can be done for the $|-\rangle$ state. Integrating out the bath, cumulant expanding the harmonic variables $\left\{y_{i}\right\}$, the partition function takes the form of

$$
\begin{gathered}
Z_{ \pm}=Z_{B} \sum_{n=0}^{\infty}\left(H_{\mathrm{if}}\right)^{2 n} \int_{0}^{\beta} d \tau_{2 n} \cdots \int_{0}^{\tau_{2}} d \tau_{1} \exp \left[\frac{1}{2} \int_{0}^{\beta} \int_{0}^{\beta} d \tau d \tau^{\prime} \sigma_{n}(\tau) \sigma_{n}\left(\tau^{\prime}\right) \tilde{C}\left(\tau-\tau^{\prime}\right)\right. \\
\left.\mp \frac{\Delta \mathcal{E}}{2} \int_{0}^{\beta} \sigma_{n}(\tau) d \tau\right]
\end{gathered}
$$

where $Z_{B}=\operatorname{Tr} \mathrm{e}^{-\beta \mathcal{H}_{B}}$ is the partition function of the bath, and

$$
\begin{aligned}
\tilde{C}\left(\tau-\tau^{\prime}\right) & =\sum_{i=1}^{N} c_{i}^{2}\left\langle y_{i}(\tau) y_{i}\left(\tau^{\prime}\right)\right\rangle_{B} \\
& =\int_{0}^{\infty} d \omega J(\omega) \frac{\hbar}{2 \pi} \frac{\cosh \left[\beta \hbar \omega / 2-\omega\left|\tau-\tau^{\prime}\right|\right]}{\sinh (\beta \hbar \omega / 2)}
\end{aligned}
$$

is the Euclidean time correlation function of the bath and the spectral density, $J(\omega)$, 
is defined as

$$
J(\omega)=\sum_{i=1}^{N} \pi \delta\left(\omega-\omega_{i}\right) \frac{c_{i}^{2}}{m_{i} \omega_{i}}
$$

Thus, the total partition function can be written in the form of a path integral over the electronic coordinate $\sigma(\tau)$

$$
Z=Z_{B} \int \mathcal{D} \sigma(\tau) \mathrm{e}^{S[\sigma(\tau)]}
$$

where

$$
\int \mathcal{D} \sigma(\tau)=\sum_{\sigma(0)= \pm 1} \sum_{n=0}^{\infty} \int_{0}^{\beta} \frac{d \tau_{2 n}}{\beta} \cdots \int_{0}^{\tau_{2}} \frac{d \tau_{1}}{\beta} .
$$

The first sum over $\sigma(0)$ is the summation over two possibilities to start a trajectory. When a trajectory starts in the $|+\rangle$ state, i.e. when $\sigma(0)=1$, it gives contribution to the reactant partition function, $Z_{+}$, otherwise, the trajectory contribute to product partition function, $Z_{-}$. The second sum is the summation over all possible number of jumps between the two states and the integrals are over all possible values of $\tau_{i}$.

The action takes the form of

$$
S[\sigma(\tau)]=2 n \ln \left(H_{\mathrm{if}} \beta\right)+\frac{1}{2} \int_{0}^{\beta} \int_{0}^{\beta} d \tau d \tau^{\prime} \sigma_{n}(\tau) \sigma_{n}\left(\tau^{\prime}\right) \tilde{C}\left(\tau-\tau^{\prime}\right)-\frac{\Delta \mathcal{E}}{2} \int_{0}^{\beta} d \tau \sigma_{n}(\tau)
$$


Written in this way the expression for the partition function, except for the unimportant factor $Z_{B}$, coincides with the partition function of a 1-D Ising model [169] with long range interactions under a external magnetic field. This is a spin system of continuously distributed spins $\sigma(\tau)$ along the "coordinate" $\tau$ and the geometry of this coordinate is a circle of length of $\beta$ due to the periodic boundary condition. The first term in the action corresponds to the action of a two-level system in which the Hamiltonian is defined as $\mathcal{H}^{\prime}$ in Eqn. 6.12 on page 120. It describes the energy of the boundaries between domains of spins with opposite orientation. This contribution arises when the two spins of opposite sign are in immediate contact and and the factor $2 n$ accounts for the number of the domain boundaries. The second term is the contribution of the influence functional. The function $\tilde{C}\left(\tau-\tau^{\prime}\right)$ describes the interaction. of two spins located at $\tau$ and $\tau^{\prime}$. It can be a short-range or long-range interaction or it can contain both, depending on whether fluctuations in the bath mode are fast or slow. Because $\tilde{C}\left(\tau-\tau^{\prime}\right) \geq 0$, the type of interaction is ferromagnetic. In the third term of the action the external magnetic field, $H$, and the magnetization of the spin system, $M$, can be identified to be

$$
H=\frac{\Delta \mathcal{E}}{2} \quad \text { and } \quad M=\int_{0}^{\beta} \frac{d \tau}{\beta} \sigma_{n}(\tau)
$$

Sum over all possible trajectories is then the sum over all configurations of the spin system. The thermal electron transfer rate of interests is the relaxation rate of the 
corresponding magnetic system from the initial state of all up $\sigma_{n}(\tau)=1$ (or all down $\left.\sigma_{n}(\tau)=-1\right)$ to the state of statistical thermal equilibrium.

To calculate the rate the partition needs to be calculated with a fixed reaction coordinate $R$. It has been suggested that the spin coordinate $\sigma(\tau)$ is a good choice for the centroid coordinate of the electron transfer [170, 171]. Therefore, in this equivalent Ising model the centroid coordinate $R$ is taken to be the magnetization $M$ which characterizes the configuration of the spin system, or equivalently, and how the electron transfer reaction makes jumps between the reactant and product states. The partition function is then written as a function of magnetization

$$
Z(M)=Z_{B} \sum_{n=0}^{\infty} g_{n}(M) \mathrm{e}^{-\beta E_{n}(M)}
$$

Here $E_{n}(M)$ is an averaged energy of a configuration with $2 n$ domains and a given magnetization $M$. The quantity $g_{n}(M)$ is a degeneracy factor which takes into account the fact that there are many ways to change the domain size leaving the magnetization of a configuration unchanged.

In obtaining the total configuration energy $E_{n}(M)$, the calculation of the interaction energy, giving by the double integral in Eqn. 6.25 on page 124 except for the factor of $1 / \beta$, is a crucial step. In the work of Stuchebrukhov and Song [145], they went beyond the so-called dilute instanton gas approximation [172] (i.e. treating the domain boundary as if they are independent, a valid assumption when the number of 
domains $2 n$ is not very large and the total magnetization is far from its extreme values \pm 1 ), by consider a configuration where all domains of spins up and spins down are of equal size. They obtained an accurate approximation for the configuration energy valid for all values of $n$ and $M$. After obtaining the expression for $Z(M)$, analytically continue it into the complex plane $M$ and integrate over the contour running from $-\mathrm{i} \infty$ to $+\mathrm{i} \infty$ according to Eqn. 6.10 on page 119, the final formula for the reaction rate takes the form

$$
\begin{aligned}
k= & \frac{1}{\beta \hbar} \sum_{n=1}^{\infty}\left(H_{\mathrm{if}} \beta\right)^{2 n} \frac{1}{n !(n-1) !} \int_{-\infty}^{+\infty} \frac{d M}{2}\left(\frac{1+M^{2}}{4}\right)^{n-1} \\
& \times \exp \left\{-\frac{\beta \Delta \mathcal{E}}{2}(1+\mathrm{i} M)-2 n \int_{0}^{\infty} \frac{d \omega}{\pi} \frac{J(\omega)}{\omega^{2}}\left[\operatorname{coth}\left(\frac{\beta \hbar \omega}{2 n}\right)-\frac{\cos \left(\frac{M \beta \hbar \omega}{2 n}\right)}{\sinh \left(\frac{\beta \hbar \omega}{2 n}\right)}\right]\right\} .
\end{aligned}
$$

Here a low temperature regime where the prefactor in Eqn. 6.10 on page 119 is $A=1 / \beta$ has been assumed. several limiting cases for the general Eqn. 6.28 will be discussed below.

\subsubsection{Limiting Cases}

\section{Single Quantum Mode Formula}

In many practical cases, Eqn. 6.28 can be further simplified by making use the fact that the low and high frequency modes in the system are well separated. The low 
frequency modes can be treated classically, and the high frequency modes quantum mechanically. If a further assumption is made that there is only a single quantum oscillator with a frequency $\omega_{\mathrm{q}}$ and all other modes are classical, Eqn. 6.28 takes a simple form [173]

$$
\begin{aligned}
& k=\frac{1}{\hbar \beta} \sum_{n=1}^{\infty} \frac{\pi^{1 / 2}}{2^{2 n-2} n !(n-1) !}\left(\beta H_{\mathrm{if}}\right)^{2 n} \sum_{m=-\infty}^{\infty}\left[\mathrm{e}^{-n S \operatorname{coth} \frac{\beta \hbar \omega_{\mathrm{q}}}{2 n}} \mathrm{I}_{|m|}(\alpha(n)) \mathrm{e}^{\frac{m \beta \hbar \omega_{\mathrm{q}}}{2 n}}\right] \\
& \times\left\{\mathrm{e}^{-\beta\left(\Delta \mathcal{E}+E_{\mathrm{rc}}+\frac{m \hbar \omega_{\mathrm{q}}}{n}\right)^{2} / 4 E_{\mathrm{rc}}} \sum_{l=0}^{n-1} \frac{\mathrm{C}_{l}^{n-1}(-1)^{l}}{\left(\beta E_{\mathrm{rc}}\right)^{\frac{l i+1}{2}}} \mathrm{H}_{2 l}(\gamma(n, m))\right\},
\end{aligned}
$$

where $S=E_{\mathrm{rq}} / \hbar \omega_{\mathrm{q}}$ and the quantities $E_{\mathrm{rc}}$ and $E_{\mathrm{rq}}$ are, respectively, the classical and quantum reorganization energies. They are related to the classical and quantum activation energy by $E_{\mathrm{rc}}=4 E_{\mathrm{ac}}$ and $E_{\mathrm{rq}}=4 E_{\text {aq }}$. The classical reorganization energy $E_{\mathrm{rc}}$ is defined by the low frequency part of the spectrum,

$$
E_{\mathrm{rc}}=\sum_{i=1}^{N_{c}} \frac{2 c_{i}^{2}}{m_{i} \omega_{i}^{2}}=\frac{2}{\pi} \int_{0}^{\omega_{c}} d \omega \frac{J(\omega)}{\omega},
$$

where $\hbar \omega_{c} \ll k_{\mathrm{B}} T$ is the upper bound of the classical frequency. The quantum reorganization energy $E_{\mathrm{rq}}$ is related to the dimensionless equilibrium coordinate shift, $\Delta$, of the quantum mode by

$$
E_{\mathrm{rq}}=\frac{1}{2} \hbar \omega_{\mathrm{q}} \Delta^{2}
$$


The quantity $S=\Delta^{2} / 2$ is a measure of the electron-vibration coupling strength. $\mathrm{I}_{|m|}(x)$ is the modified Bessel function, $\alpha(n)=n S / \sinh \left(\beta \hbar \omega_{\mathrm{q}} / 2 n\right), \mathrm{C}_{l}^{n-1}$ is the bino-

mial coefficient, $\mathrm{H}_{2 l}(x)$ is the Hermite polynomial, and $\gamma(n, m)=\left[\frac{\beta\left(\Delta \mathcal{E}+m \hbar \omega_{\mathrm{q}} / n\right)^{2}}{4 E_{\mathrm{rc}}}\right]^{\frac{1}{2}}$. This is a useful formula that will be used to study the effect of adiabaticity and quantum correction.

\section{Nonadiabatic Case}

The sum over $n$ in Eqn. 6.28 and Eqn. 6.29 is a sum over all perturbation orders in the coupling $H_{\text {if }}$. When $H_{\text {if }}$ is small, only the first term of the expansion needs to be taken into account. The $n=1$ term in Eqn. 6.28 coincides exactly with the well-know exact quantum mechanical expression for the nonadiabatic rate $[146,151]$.

A more standard expression is obtained from the $n=1$ term in Eqn. 6.29:

$$
k=\left(\frac{\pi \beta}{\hbar^{2} E_{\mathrm{rc}}}\right)^{1 / 2} H_{\mathrm{if}}^{2} \sum_{m=-\infty}^{\infty}\left[\mathrm{e}^{-S \operatorname{coth} \frac{\beta \hbar \omega_{\mathrm{q}}}{2}} \mathrm{I}_{|\mathrm{m}|}(\alpha(1)) \mathrm{e}^{\frac{\mathrm{m} \beta \hbar \omega_{\mathrm{q}}}{2}}\right] \mathrm{e}^{-\beta\left(\Delta \mathcal{E}+E_{\mathrm{rc}}+m \hbar \omega_{\mathrm{q}}\right)^{2} / 4 E_{\mathrm{rc}}}
$$

For a low temperature such that $\beta \hbar \omega_{\mathrm{q}} \gg 1, \alpha(1) \ll 1$, the above formula takes the most popular form in the literature [174]

$$
k=\left(\frac{\pi \beta}{\hbar^{2} E_{\mathrm{rc}}}\right)^{1 / 2} H_{\mathrm{if}}^{2} \sum_{m=0}^{\infty}\left[\mathrm{e}^{-S} \frac{1}{m !} S^{m}\right] \mathrm{e}^{-\beta\left(\Delta \mathcal{E}+E_{\mathrm{rc}}+m \hbar \omega_{\mathrm{q}}\right)^{2} / 4 E_{\mathrm{rc}}}
$$

where the terms with negative $m$ 's were omitted because of containing an additional factor $\exp \left(-|m| \beta \hbar \omega_{\mathrm{q}}\right)$. This formula corresponds to the case that the reactant is in 
its vibrational ground state, but the product may be produced in higher vibrational states. By comparing to the golden-rule rate expression with all bath modes being classical

$$
k=\left(\frac{\pi \beta}{\hbar^{2} E_{\mathrm{rc}}}\right)^{1 / 2} H_{\mathrm{if}}^{2} \mathrm{f}^{-\beta\left(\Delta \mathcal{E}+E_{\mathrm{rc}}\right)^{2} / 4 E_{\mathrm{rc}}}
$$

we can see the effects of the quantum mode in Eqn. 6.33. The rate is a weirhted sum of all possible transitions to the final vibrational excited states. The quantity $m$ is the total vibrational quanta involved in the process, i.e. $m \hbar \omega_{\mathrm{q}}$ is the vibrational energy of the initial state minus the final state. The terms in the square bracket is a Franck Condon nuclear overlap function. It is the square of the overlap integral of the ground and $m$ 'th level harmonic oscillator wavefunctions in the 'classical forbidden' region. That is, the reorganization of the quantum mode occurs through a subbarrier (tunneling) motion.

Equation 6.33 is widely used by experimentalists to study electron transfer reactions. For example, Closs and Miller [174] applied it in the experimental verification of the existence of Marcus inverted region [147]. Since Eqn. 6.33 is just a limiting case of Eqn. 6.29, Eqn. 6.29 is tested by reproduce the calculation in Closs and Miller's work (Fig. 6.11) and then used to examine the applicability of the usual nonadiabatic theory as shown in the next section. It is clear in Fig. 6.11 that the electron transfer rate goes through an inverted region as the thermodynamic driving force increases. 

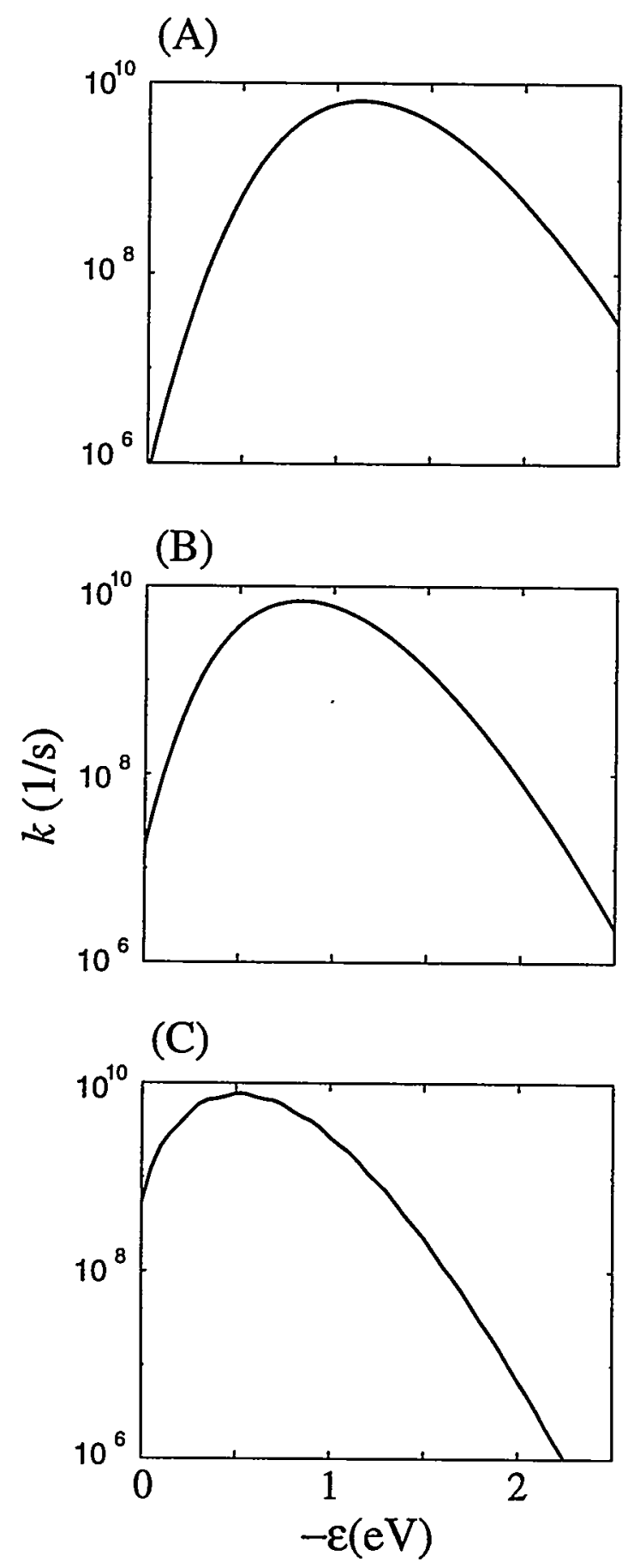

Figure 6.11: Testing Eqn. 6.29 by reproducing the calculation in Closs and Miller's work [174]. Parameters are $H_{\mathrm{if}}=6.2 \mathrm{~cm}^{-1}, \omega_{\mathrm{q}}=1500 \mathrm{~cm}^{-1}, E_{\mathrm{rq}}=0.45 \mathrm{eV}$. The value of $E_{\mathrm{rc}}$ are (A) 0.75 , (B) 0.45 , and (C) $0.15 \mathrm{eV}$. 
The substantial difference from the classical prediction of an inverted parabola results from the effect of nuclear tunneling which is more pronounced in the inverted region.

\section{Effects of Coupling Strength in the Normal and Inverted Regions}

The main purpose of this calculation is to examine the contributions of the higher order terms $(n>1)$ in Eqn. 6.29 on page 128 in the normal and inverted regions. These terms should be important for strong coupling system. Figure 6.12 shows the calculation with the parameters $E_{\mathrm{rq}}=0.45 \mathrm{eV}, E_{\mathrm{rc}}=0.75 \mathrm{eV}, \omega_{\mathrm{q}}=1500 \mathrm{~cm}^{-1}$ and $T=300 \mathrm{~K}^{2}$.

There are two interesting features. First, in both regions, the reaction rate initially increases quadratically for small couplings, as predicted by the nonadiabatic formula, but then develops big deviation as the coupling becomes stronger. In the normal region, the rate is higher than the nonadiabatic prediction and monotonically increases with the coupling. In the inverted region, however, the rate is lower than the nonadiabatic prediction and it reaches maximum and then decreases with further increase of the coupling. This behavior is a reflection of the fact that large electronic coupling causes the separation of the adiabatic surfaces (Fig. 6.12). The increase in surface separation lowers the barrier for the normal region, increases the chance of staying in the lower surface, and enhances the rate. Electron transfer in the inverted

\footnotetext{
${ }^{2}$ In Ref. [145] a similar calculation is done for the inverted region. However, the result in Ref. [145] can not be reproduced by using their reported parameters. There may have been typographical errors in that paper. Even so, the trends described in that paper is verified.
} 
(A) Normal region
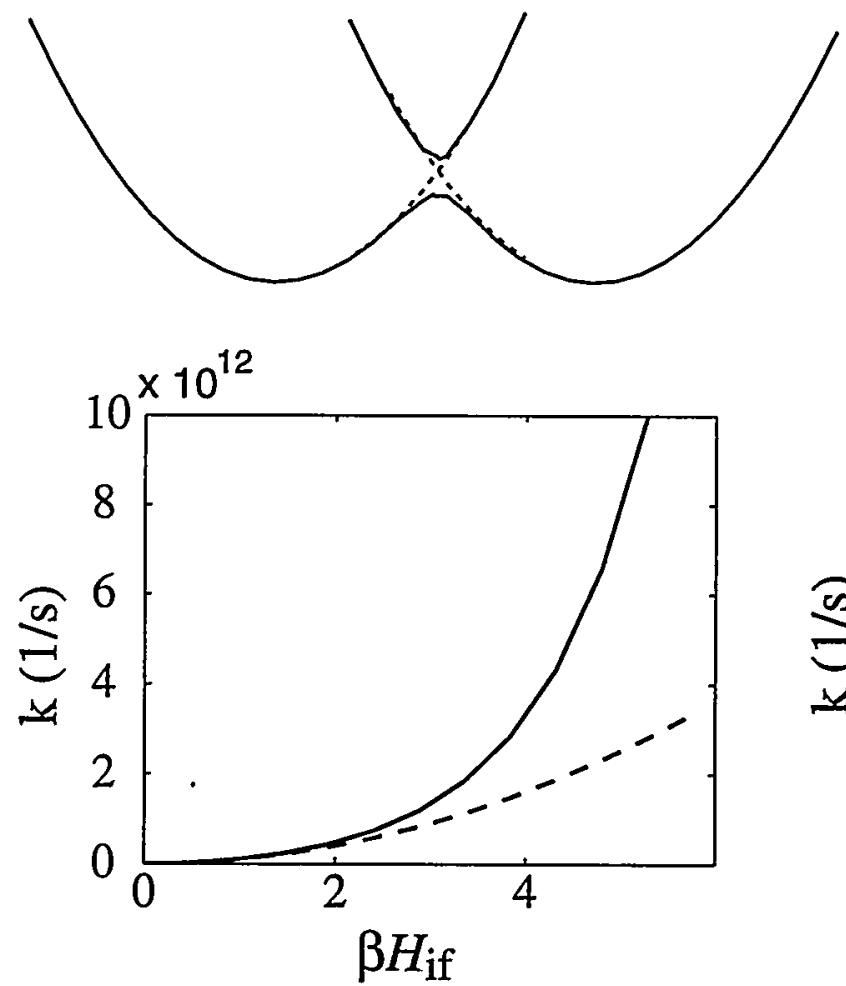

(B) Inverted region
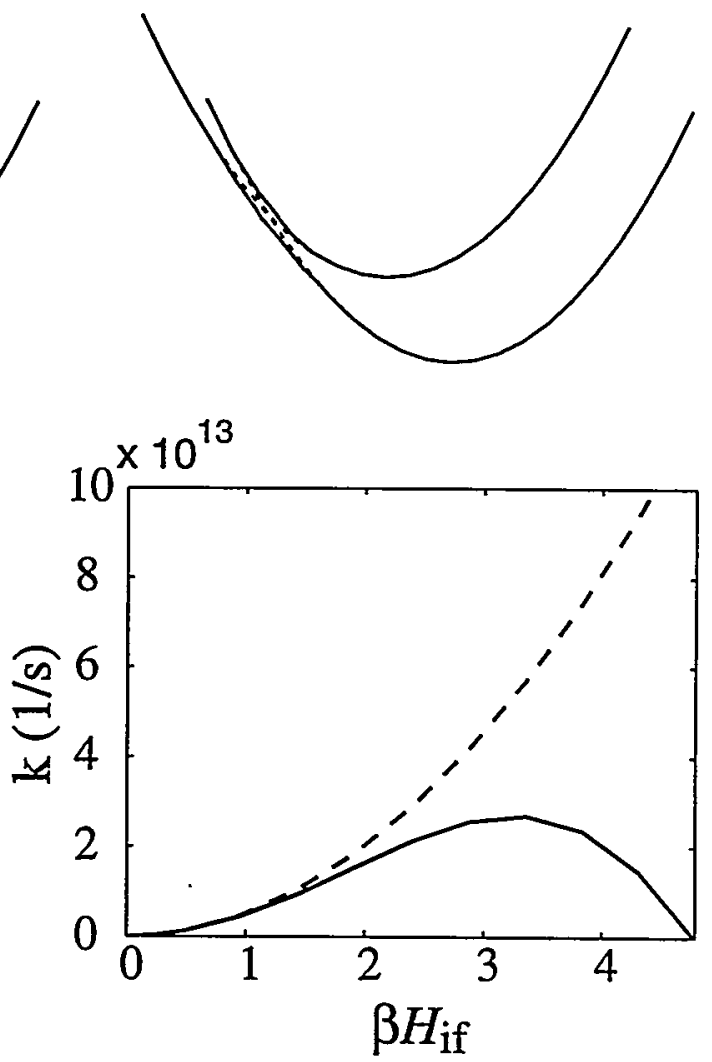

Figure 6.12: Reaction rate as a function of electron coupling $\beta H_{\text {if }}$ in the (A) normal region, $-\Delta \mathcal{E}=0.3 \mathrm{eV}$, and the (B) inverted region, $-\Delta \mathcal{E}=1.45 \mathrm{eV}$. Other parameters are $E_{\mathrm{rq}}=0.45 \mathrm{eV}, E_{\mathrm{rc}}=0.75 \mathrm{eV}, \omega_{\mathrm{q}}=1500 \mathrm{~cm}^{-1}$ and $T=300 \mathrm{~K}$. Solid lines are the rate calculated by Eqn. 6.29. Dashed lines are the $n=1$ nonadiabatic terms. 
region, however, has to cross between the surfaces. The increase in surface separation makes the crossing harder, competes with the enhancement due to stronger coupling, eventually overwhelms, and makes the rate decrease with coupling. Second, the nonadiabatic formula appears to be applicable not only for $H_{\text {if }} \beta \ll 1$ but also for a pretty wide range of coupling strength. With the above parameters the deviation from the full rate is about $4 \%$ for $H_{\text {if }} \beta \sim 1$ in both the normal and inverted regions. Equation 6.29 can be used to study the relative contribution from each $n$ term, and hence determine the adiabaticity of the reaction.

\section{Resonant Effects}

If the same parameters as those in Fig. $6.11 \mathrm{C}$ are used and the temperature is lowered from $298 \mathrm{~K}$ to $50 \mathrm{~K}$, an oscillatory dependence of rate on the thermodynamic driving force is revealed (Fig. 6.13). This peculiar dependence comes from the inclusion of a single quantum mode in the model. Here the first maximum corresponds to the usual classical inverted region due to the classical modes $\left(-\Delta \mathcal{E}=E_{\mathrm{rc}}\right)$. The following maxima come from a resonant effect due to the excitation of the quantum mode. Whenever the system has an energy high enough to excite a vibrational quanta, it can dump its energy to this mode more effectively due to resonance, and the rate is enhanced. These oscillations are analogous to vibrational structure of optical electronic transitions and they exist under the conditions of high $\hbar \omega_{\mathrm{q}}$, low $E_{\mathrm{rc}}$, and low $T$, i.e. when $\hbar \omega_{\mathrm{q}}>\left(4 E_{\mathrm{rc}} k_{\mathrm{B}} T\right)^{\frac{1}{2}}$ (see Eqn. 6.33 and 6.29). The interval between 

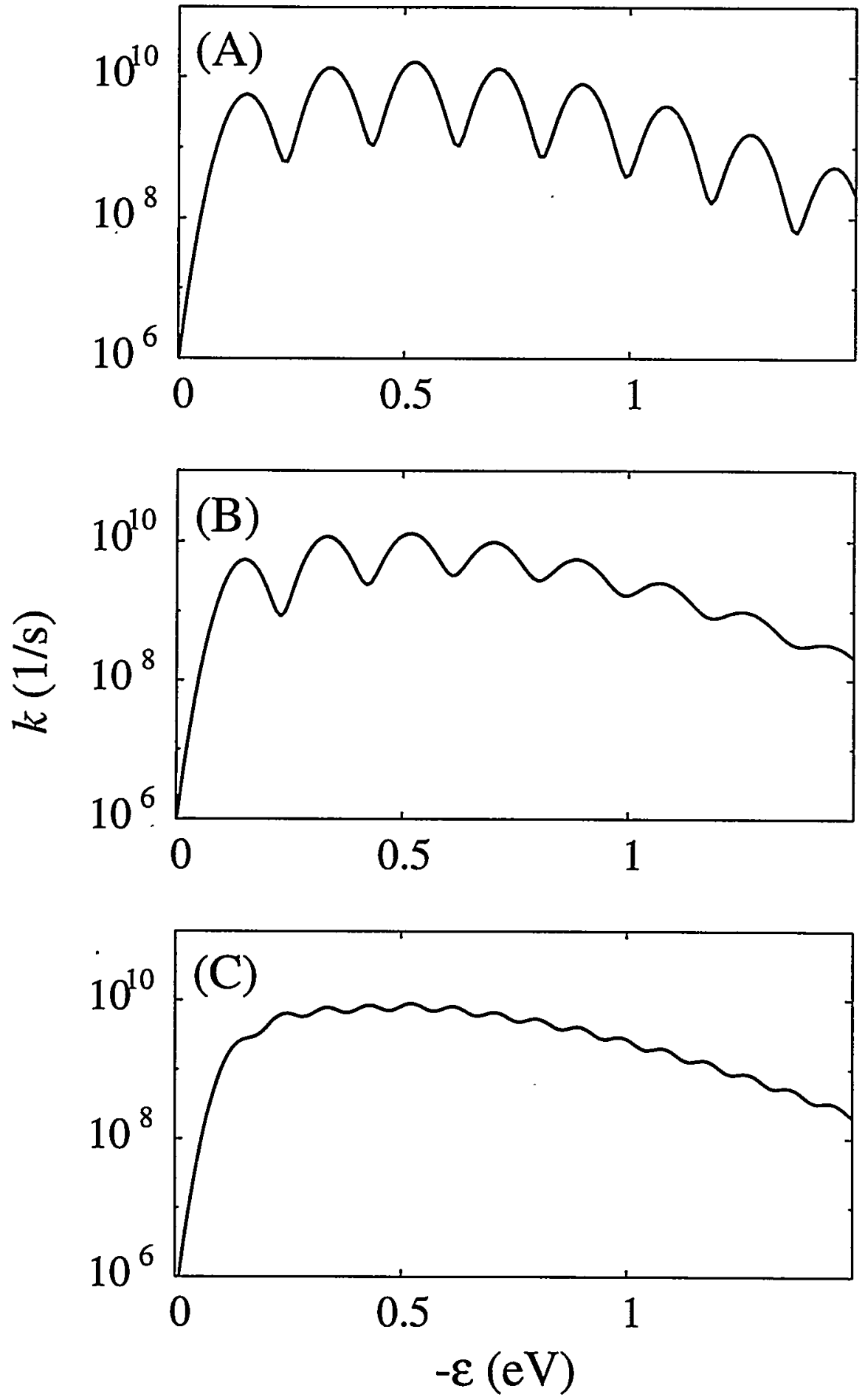

Figure 6.13: Oscillatory dependence of rate on the thermodynamic driving force. (A) Single quantum mode of frequency $1500 \mathrm{~cm}^{-1}$ : (B) Two quantum modes of frequencies 1650 and $1350 \mathrm{~cm}^{-1}$. (C) Two quantum modes of frequencies 2250 and $750 \mathrm{~cm}^{-1}$ with the same reorganization energy. 
maxima is $\hbar \omega_{\mathbf{q}}$. When the temperature is high or there exist more than two modes with different frequencies, the oscillatory behavior will be smeared out $[146,175]$. Figure 6.13 demonstrates the erosion of oscillations by including a second quantum mode of a different frequency.

\subsubsection{Remarks}

The work of Stuchebrukhov and Song represents one of the most recent development in the study of strongly interacting systems. The combination of the ideas of a complex partition function and a complex centroid reaction coordinate yields a general explicit formula for the rate valid in all orders of perturbation theory in electronic coupling. Inevitably, there are still many limitations on the present theory. For example, the results are only an approximation which is valid as long as Eqs. 6.86.10 on page 119 are valid. In fact, this might be the most catchy point in this way of calculating reaction rate, because the idea of a complex free energy, though natural enough for metastable systems, seems rather artificial for a system like the symmetric double well, where the full free energy must be real [166]. This problem should be addressed in the future. 


\subsection{Analysis of Self-Trapping Dynamics}

By inspecting the potential surfaces of the free and self-trapped states (Fig. 6.7 on page 108) and making an analogy between electron self-trapping processes and electron transfer reactions (Sect. 6.5), the strong $k_{\|}$-dependence of the self-trapping rate (Fig. 6.3A on page 98) can be understood and quantitatively modeled. Curves in the $V_{\mathrm{f}}$ manifold represent the different initial electronic states (in zero order) with translational symmetry labelled by $k_{\|}, V_{\mathrm{s}}$ represents the final state, and the lattice distortion $Q$ is the reaction coordinate. A self-trapping process starting from an initial state of particular $k_{\| \mid}$and band energy $E_{\| \mid}$corresponds to a reaction with exothermicity of $-\Delta \mathcal{E} \equiv E_{\|}+E_{\text {st }}$. The extent of the mixing between the initial and final states at the crossing point is determined by the matrix element $H_{\mathrm{fs}}$ which is the electronic coupling of the two states. Within the displaced harmonic potential approximation, assuming that the electronic coupling is independent of $k_{\|}$and weak $\left(H_{\mathrm{fs}} \ll k_{\mathrm{B}} T\right)$, and the system has a thermal energy higher than the nuclear vibrational energy, i.e. in the classical nonadiabatic limit, the rate is given by (see Eqn. 6.34) $[144,146,176]$

$$
k_{\mathrm{st}}=\left(\frac{\pi}{\hbar^{2} E_{\mathrm{rel}} k_{\mathrm{B}} T}\right)^{\frac{1}{2}} H_{\mathrm{fs}}^{2} \mathrm{e}^{-\left(\Delta \mathcal{E}+E_{\mathrm{rel}}\right)^{2} / 4 E_{\mathrm{rel}} k_{\mathrm{B}} T}
$$

and goes through the so-called Marcus inverted region when the exothermicity is equal to the lattice relaxation energy, $-\Delta \mathcal{E}=E_{\text {rel }}$.

The model discussed above treats the nuclear motion classically, a valid approach 
in the high temperature limit. At lower temperatures, the quantum-mechanical nature of nuclear motion needs to be taken into account. The lattice can go through the self-trapping barrier via tunneling. For small polaron formation in a molecular lattice the electron can interact with both intra- and intermolecular vibrations, forming molecular and lattice polarons, respectively. The interaction of an electron with intermolecular vibrations involves the displacement of molecules from equilibrium positions. Vibrational frequencies of these modes are very low, for example, $\sim 45 \mathrm{~cm}^{-1}$ for the Debye energy of a n-heptane layer ${ }^{3}$ It is adequate to treat them classically at our experimental temperature, $120 \mathrm{~K}$. The interaction with intramolecular vibrations involves changes in bond lengths and bond angles. At $120 \mathrm{~K}$ these high-frequency intramolecular modes will be frozen and require a quantum-mechanical treatment. To avoid introducing too many fitting parameters, we made the simplest assumption that self-trapping involves a single quantum-mechanical intramolecular mode with a frequency $\omega_{\mathrm{q}}$ and a reorganization energy $E_{\mathrm{rq}}$, and all other intermolecular modes are classical with a reorganization energy $E_{\mathrm{rc}}$. Without knowing the strength of the electronic coupling, $H_{\mathrm{fs}}$, it is hard to determine whether the process is adiabatic or non-adiabatic. Therefore, we analyzed the data using Eqn. 6.29 on page 128 which is the rate expression includes all perturbation orders within the single quantum mode assumption.

\footnotetext{
${ }^{3}$ The Debye frequency for a 2-D n-heptane lattice is estimated from the speed of sound and the surface mesh dimensions [82]. The speed of sound for solid n-heptane was inferred from the liquid measurements in Ref. [177] using the density of the solid.
} 
Figure 6.14A shows the self-trapping rates obtained for a series of photoemission angles at $120 \mathrm{~K}$ and plotted versus the exothermicity of the process [66]. In this analysis we attributed the delocalized state decay rate (after subtracting a rate for tunneling back into the metal which is independent of $k_{\|}$) to self-trapping. Exothermicity $-\Delta \mathcal{E}$ is determined by the photoelectron kinetic energy difference between the delocalized and localized states (see Fig. 6.2 on page 97). The solid line is a best fit of the data with parameters $H_{\mathrm{fs}}=91 \mathrm{~cm}^{-1}, E_{\mathrm{rc}}=0.057 \mathrm{eV}, E_{\mathrm{rq}}=0.18 \mathrm{eV}$, and $\hbar \omega_{\mathrm{q}}=750 \mathrm{~cm}^{-1}$. It is important to note that the first order term in Eqn. 6.29 is an exact expression for a quantum nonadiabatic process, and it accounts for nearly $96 \%$ of the fit in Fig. 6.14A. That is, this process at $120 \mathrm{~K}$ is reasonably well described by the nonadiabatic theory. For a comparison to the classical theory for nuclear motion, we also fit the data with Eqn. 6.35. A good fit is only obtained by making the temperature an adjustable parameter. However, the resulting temperature, $280 \mathrm{~K}$, is significantly higher than the experimental temperature, $120 \mathrm{~K}$. Therefore, we conclude that the formation of the 2-D small polaron is governed by quantum dissipative processes such as tunneling, and the classical theory is not applicable to this system.

It is encouraging that the parameters extracted from the fit are all consistent with known values for polarons in other molecular solids. In organic molecular crystals an electron interacts with the intermolecular vibrations via the charge-induced dipole interaction which is weak and short range. This interaction manifests itself chiefly through dynamic modulation of the polarization energy. This modulation is of the 

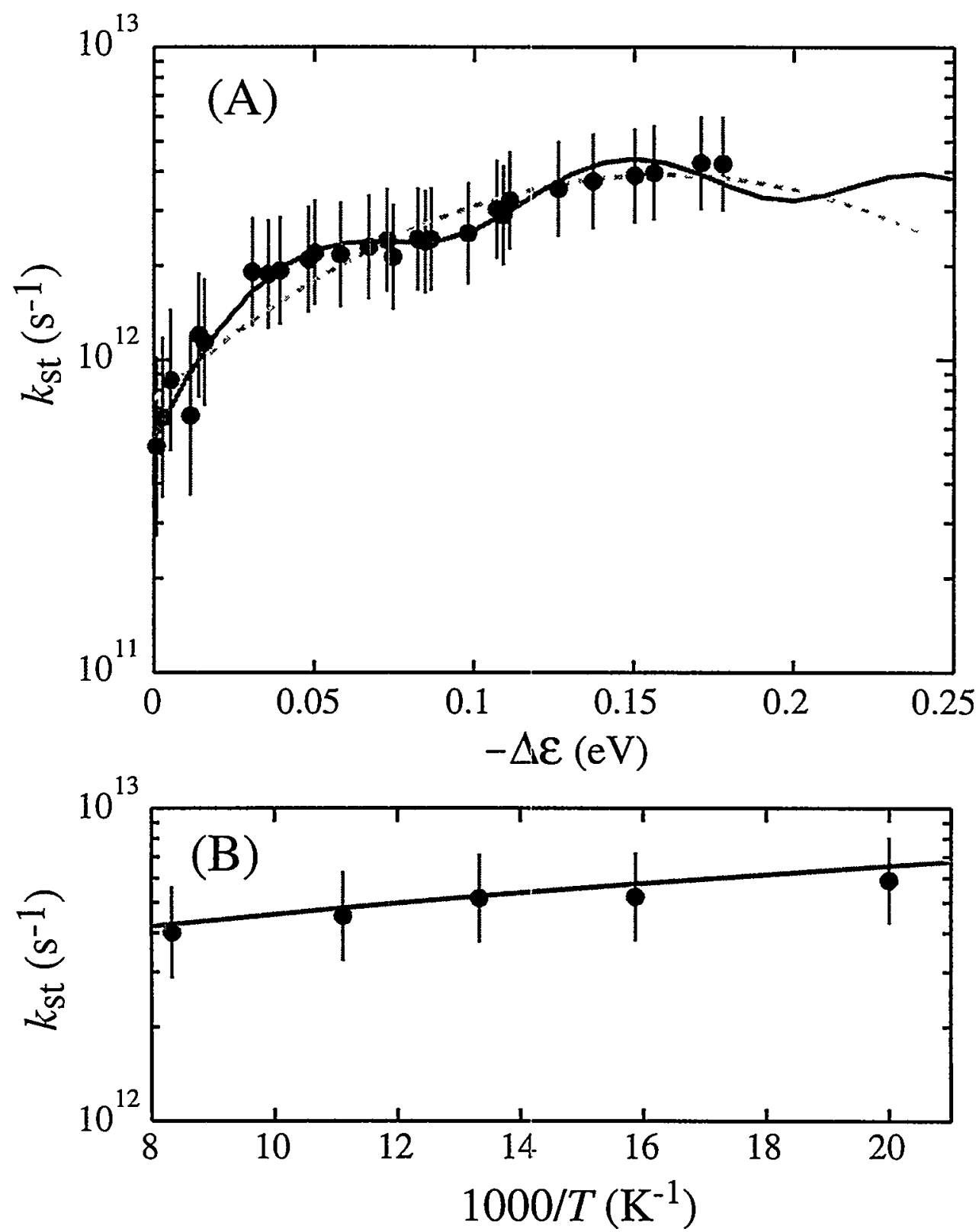

Figure 6.14: (A) Logarithmic plot of self-trapping rate of the delocalized state versus exothermicity for a bilayer at $120 \mathrm{~K}$. The $95 \%$ confidence limit for each data point is indicated by the vertical line. The solid line was computed by Eqn. 6.29 with the parameters $H_{\mathrm{fs}}=91 \mathrm{~cm}^{-1}, E_{\mathrm{rc}}=0.057 \mathrm{eV}, E_{\mathrm{rq}}=0.18 \mathrm{eV}$, and $\hbar \omega_{\mathrm{q}}=750 \mathrm{~cm}^{-1}$. The dashed line was computed by classical theory (Eqn. 6.35) with the parameters $H_{\mathrm{fs}}=77 \mathrm{~cm}^{-1}, E_{\text {rel }}=0.16 \mathrm{eV}$, and $T=280 \mathrm{~K}$. (B) Temperature dependence of self-trapping rates for a bilayer at $18^{\circ}\left(k_{\|}=0.21 \AA^{-1}\right)$. The solid line was computed by Eqn. 6.29 with $-\Delta \mathcal{E}=0.14 \mathrm{eV}$ and the same parameters as in (A). 
order of $0.03 \mathrm{eV}[178]$ and is consistent with the reorganization energy of $E_{\mathrm{rc}}=$ $0.057 \mathrm{eV}$. The determined $E_{\mathrm{rq}}=0.18 \mathrm{eV}$ is similar to the $0.15 \mathrm{eV}$ effective formation energy of a molecular polaron in polyacene crystals [178].

The high-frequency mode $\hbar \omega_{\mathbf{q}}=750 \mathrm{~cm}^{-1}$ suggests that an in-phase methylene rocking mode [179] is involved in forming the polaron. These modes are not thermally excited and they proceed from their initial to their final state equilibrium value by a subbarrier (tunneling) motion. The reorganization energy $E_{\mathrm{rq}}$ is related to the dimensionless equilibrium coordinate shift $\Delta$ by $E_{\mathrm{rq}}=\frac{1}{2} \hbar \omega_{\mathrm{q}} \Delta^{2}$. The oscillatory dependence of $k_{\mathrm{st}}$ on $-\Delta \mathcal{E}$ (Fig. $6.14 \mathrm{~A}$ ) comes from the inclusion of a single quantum-mechanical mode in the model. The first maximum corresponds to the usual classical inverted region resulting from the intermolecular modes $\left(-\Delta \mathcal{E}=E_{\mathrm{rc}}\right)$. Subsequent maxima originate from a resonant effect resulting from the excitation of the intramolecular mode [175] and the interval between maxima is $\hbar \omega_{\mathrm{q}}$ (see Fig. 6.14A).

With the use of the above parameters, the predicted and measured $k_{\mathrm{st}}$ for $\Delta \mathcal{E}=$ $-0.14 \mathrm{eV}$ are plotted as a function of temperature in Fig. 6.14B. The rates are very weakly dependent on temperature because of nuclear tunneling in the high-frequency modes, and this non-Arrhenius temperature dependence is fairly well reproduced by the theory. It should be note that the higher order terms become more important at lower temperatures. The first order term accounts for only $83 \%$ of the rate for the data point at $50 \mathrm{~K}$.

A comparison between $E_{\text {loc }}$ and $E_{\text {rel }}$ can tell us the relative strength of delocaliza- 
tion versus localization. Within the isotropic $2-\mathrm{D}$ tight binding approximation, $B$ is related to $m^{*}$ by $B=2 \hbar^{2} / m^{*} a^{2}$, where $a$ is the lattice constant. With $m^{*}=1.2 m_{\mathrm{e}}$ for the delocalized state, an estimation of $E_{\text {loc }}=0.25 \mathrm{eV}$ is obtained ${ }^{4}$. However, this approach may result in an overestimation $(\sim 1.5$ times) of the electron kinetic energy of strongly localized states [142]. The localization energy is thus comparable to the lattice relaxation energy $\left(E_{\mathrm{rel}}=E_{\mathrm{rc}}+E_{\mathrm{rq}}=0.23 \mathrm{eV}\right)$. This explains why the self-trapping energy (given by Eq. 6.3) is small for this system.

\subsection{Beyond the Single Quantum Mode}

\section{Assumption}

The application of the electron transfer theory under the single quantum mode assumption to model the electron self-trapping dynamics has been quite successful. The energy dependence of self-trapping rate at $120 \mathrm{~K}$ and the non-Arrhenius temperature dependence at $\Delta \mathcal{E}=-0.14 \mathrm{eV}$ are well reproduced with reasonable parameters. The assumption of single quantum mode, however, may be oversimplified. Naturally, there may be multiple quantum modes involved in self-trapping. In fact, theoretical calculation have shown that vibrational frequencies of many n-alkane molecules, in-

\footnotetext{
${ }^{4}$ Because of the three-fold symmetry of $\mathrm{Ag}(111)$ surface, n-heptane layers grown on $\mathrm{Ag}(111)$ can form domains with surface mesh axis in each domain being related to each other by $120^{\circ}$ rotation. The measured effective mass is an average value over different domains. For a crude estimation of $B$, we assume a square lattice of lattice constant $a$ that gives the same area as the n-heptane surface mesh dimensions.
} 
cluding $\mathrm{n}$-heptane, span a wide range from 1480 to $40 \mathrm{~cm}^{-1}$ [179]. Aside from that possibility, if only one intramolecular mode behaves quantum mechanically at $120 \mathrm{~K}$, as the temperature is lowered further, many modes in the continuous vibrational spectrum of the intermolecular modes that originally behave classically may now behave quantum mechanically and need proper treatment. At low temperatures and for strongly exothermic processes it is essential to consider explicitly the effect of the medium frequency distribution because the reorganization of quantum modes are important in these regions [146]. In this section a brief description of how to introduce the collective medium response into the rate expression is presented.

The theoretical treatment of frequency dispersion was carried out first by Ovchinnikov and Ovchinnikova $[180,151]$ and was investigated further by Dogonadze and coworkers $[181,182]$. Along the same line as Ref. $[146,183]$, consider the electrostatic interaction between the electrons and a continuous dielectric medium. The response of the dielectric to the electric field is defined by the dielectric function $\epsilon(\omega)$. Treating the medium as a collection of harmonic oscillators, the dielectric polarization of the medium is then described by the equilibrium positions of oscillators. When electron transfer reactions or electron self-trapping processes occur, the changes in electric fields from the initial state to the final state induce-a shift in the equilibrium position, $q_{i 0}$, of the dielectric polarization oscillator. This shift is related to the dielectric 
constant $[146]$

$$
\frac{1}{2} \sum_{i} \hbar \omega_{i} q_{i 0}^{2}=\frac{\int d \mathbf{r}\left|D^{i}-D^{f}\right|^{2}}{4 \pi^{2}} \frac{\operatorname{Im} \epsilon(\omega)}{|\epsilon(\omega)|^{2}}
$$

where $D^{i}$ and $D^{f}$ are the dielectric displacement vectors of the electrons in the initial and final states. Here the spatial dispersion, i.e. the dependence of $\epsilon$ on the wave vector, has been neglected. The left hand side of Eqn. 6.36 represents the reorganization energy $E_{\mathrm{r}}$ [146]. As discussed in Sect. 6.5.4, in the spin-boson model $E_{\mathrm{r}}$ is related to the spectral density $J(\omega)$ by [155]

$$
E_{\mathrm{r}}=\int_{0}^{\infty} \frac{2}{\pi} \frac{d \omega}{\omega} J(\omega)
$$

Comparing Eqn. 6.36 and Eqn. 6.37, the spectral density can be written as [183]

$$
J(\omega)=\frac{\int d \mathbf{r}\left|D^{i}-D^{f}\right|^{2}}{8 \pi} \frac{\operatorname{Im} \epsilon(\omega)}{|\epsilon(\omega)|^{2}}
$$

This result is valid under linear response theory [151]. In conjunction with Eqn. 6.28, one now has the full expression for the rate constant in terms of the entire dielectric spectral response.

If the experimentally measured $\epsilon(\omega)$ is available, the calculation of the rate will become straightforward. In fact, this idea has been applied to calculate the ferrousferric electron transfer rate in water [183]. There are also recent reports on the 
calculations of time-dependent Stokes shift and fluorescence spectra for the solvation of photoexcited coumarin in polar solvents $[184,185]$ based on experimental $\epsilon(\omega)$. The application of this model to the 2-D electron self-trapping studied here should also be fruitful except that the available $\epsilon(\omega)$ data were found only in the range of $0.3-300 \mathrm{~cm}^{-1}$ for liquid $\mathrm{n}$-heptane $[186,187]$. The dielectric-loss factor as a function of logarithmic frequency shows two separate peaks. The broad peak centered at $3 \mathrm{~cm}^{-1}$ corresponds to Debye absorption, while the stronger peak centered at $75 \mathrm{~cm}^{-1}$ corresponds to Poley absorptions. If $\epsilon(\omega)$ of $\mathrm{n}$-heptane solids also exhibits absorption in this or even higher frequency range, as our experimental temperature is lowered to $50 \mathrm{~K}$, these modes will behave quantum mechanically. The single quantum mode assumption and the assumption that classical modes and quantum modes are well separated in energy are no longer valid. This may explain why the single quantum mode calculation can not reproduce the dependence of $k_{\mathrm{st}}$ on $-\Delta \mathcal{E}$ at $50 \mathrm{~K}$ (Fig. 6.15) because it predicts a oscillatory dependence with a much larger amplitude than is observed experimentally. In the presence of many quantum modes, the oscillation will be smeared out.

Clearly, it will be useful to obtain dielectric data for n-alkane solids and extend the measurement to higher frequency. However, a proper theory for 2-D electron self-trapping at ultrathin n-alkane layers requires more than bulk dielectric data. An equation to calculate $\epsilon(\omega)$ for a 2-D slab from the bulk $\epsilon(\omega)$ is needed. For an isotropic 3-D system, the Clausius-Mossotti relation can be utilized to obtain the polarizability 


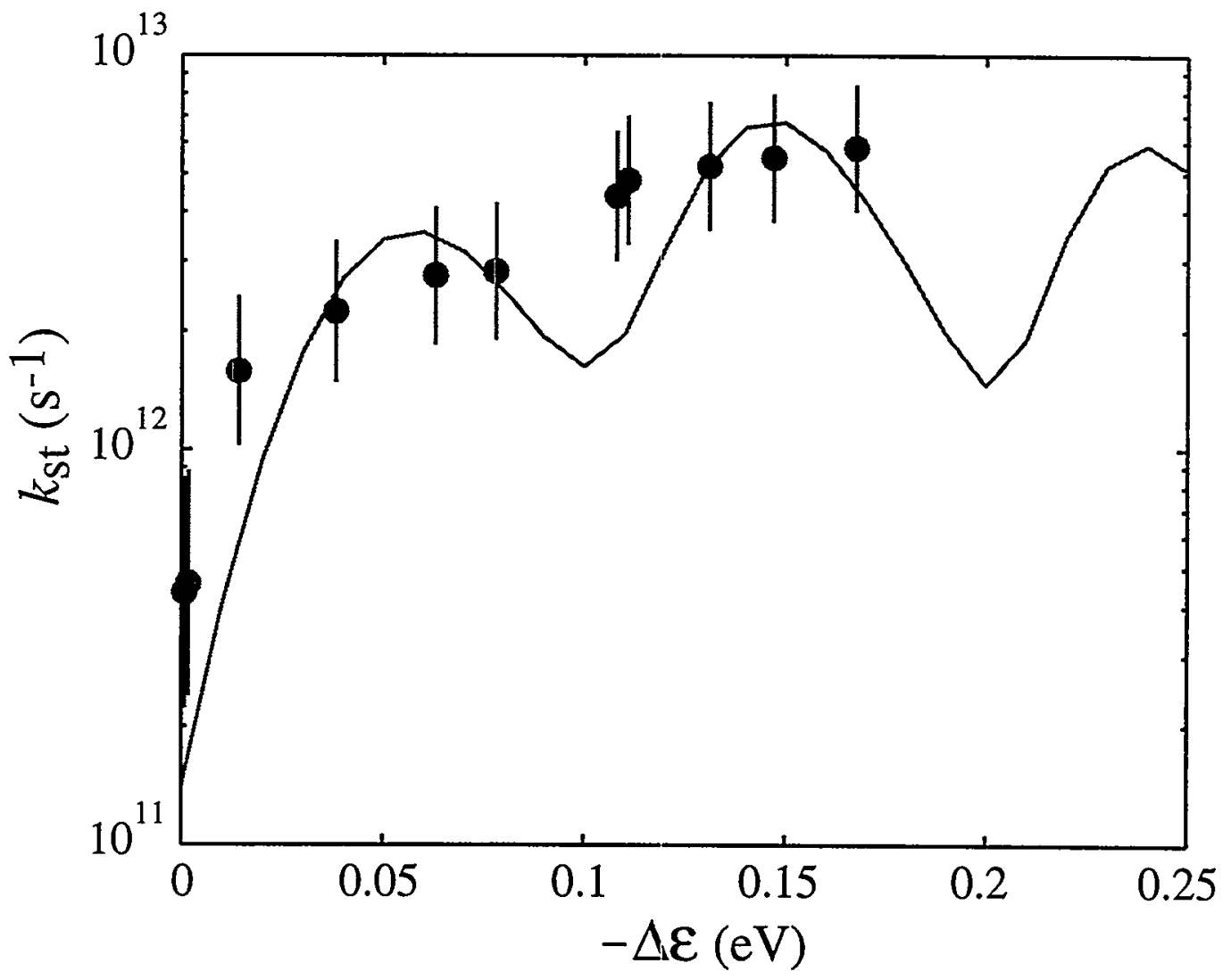

Figure 6.15: (A) Logarithmic plot of self-trapping rate of the delocalized state versus exothermicity for a bilayer at $50 \mathrm{~K}$. The $95 \%$ confidence limit for each data point is indicated by the vertical line. The solid line was computed by Eqn. 6.29 on page 128 with the same parameters as in Fig. 6.14 on page 140A. 
$\alpha(\omega)$ from bulk dielectric constant:

$$
\alpha(\omega)=\frac{3}{4 \pi N} \frac{\epsilon(\omega)-1}{\epsilon(\omega)+2} .
$$

It has been shown that the Clausius-Mossotti equation can be derived from a coarsegrained Gaussian model of a dielectric material $[188,189]$. Extending the derivation into a square grid of polarizable cells should give an equation suitable to link $\alpha(\omega)$ to the dielectric constant for a 2-D slab. The molecular nature of the medium can be treated by including the spatial dispersion of the dielectric constant, $\epsilon(\mathbf{k}, \omega)$. However, the experimental data for $\epsilon(\mathbf{k}, \omega)$ are usually not available. 


\section{Chapter 7}

\section{Conclusion}

The study of excited electron dynamics by femtosecond time- and angle-resolved TPPE has provided important insight into the electronic processes at metal-dielectric interfaces. For the bare metal surface, image state binding energies and lifetimes are dominated by their position with respect to the projected bulk band gap. Physisorption of dielectric overlayers significantly alters the behavior of image states. The work within this dissertation emphasizes two aspects of these effects and addresses the questions posted in Chapter 1.

First, we monitored the layer-by-layer evolution of the interfacial electronic structure and dynamics. Such a study provides information on the nature of the interfacial potential. It is found that the electron affinity of the overlayer material strongly affects the dynamics and energies of the interfacial excited states. The formation of a tunneling barrier or potential well at the interface due to the presence of the adlayer 
plays a very important role in determining the spatial distribution of the interfacial electrons as well as the electronic coupling across the interface.

We have also shown that an initially delocalized electron at the metal-alkane interface undergoes localization within a few hundred femtoseconds. The mechanism for localization is attributed to 2-D self-trapping. The analysis based on electron transfer theory reveals that small polaron formation involves the inter- and intramolecular vibrational modes of the overlayer, and their contributions to the lattice relaxation energy has been determined. The non-Arrhenius temperature dependence of the selftrapping rate indicates a strong quantum contribution. This study demonstrates that the ability to momentum-resolve the dynamics for a wide range of delocalized states provides detailed experimental information for the self-trapping process. Such information is usually inaccessible with transient absorption or other purely optical techniques that have been used to time-resolve self-trapping dynamics [190, 124].

Recently, the work by other members of the Harris group has extended to more reactive adsorbates, such as conjugated hydrocarbons, polar molecules, and chemisorbing self-assembled monolayers. These studies will further our understanding on the electronic processes for a wide variety of metal-dielectric interfaces. The best use of the results presented here and those still to come would be to test a sophisticated model of the metal-dielectric interface which takes into account the molecular parameters, realistic potentials near the interfaces, and the interaction of the layer with the metal. The energy, effective mass, and lifetime data will provide a rigorous test of such a model. 


\section{Bibliography}

[1] P. Avouris and R. E. Walkup, Annu. Rev. Phys. Chem. 40, 173-206 (1989).

[2] J. R. Sheats, H. Antoniadis, M. Hueschen, W. Leonard, J. Miller, R. Moon, D. Roitman, and A. Stocking, Science. 273, 884-888 (1996).

[3] Surface Science: The First Thirty Years, C. B. Duke, ed., (North-Holland, Amsterdam, 1994).

[4] F. J. Himpsel, Surf. Sci. Rep. 12, 1-48 (1990).

[5] R. J. Hamers, Annu. Rev. Phys. Chem. 40, 531-559 (1989).

[6] P. Avouris, Account. Chem. Res. 27, 159-165 (1994).

[7] K. Giesen, F. Hage, F. J. Himpsel, H. J. Riess, and W. Steinmann, Phys. Rev. Lett. 55, 300-3 (1985).

[8] T. Fauster and W. Steinmann, "Two-photon photoemission spectroscopy of Image states," in Photonic Probes of Surfaces, P. Halevi, ed., (Elsevier, Amsterdam, 1995), pp. 347-411. 
[9] I. E. Tamm, Phys. Z. Sowjetanion 1, 733 (1932).

[10] A. W. Maue, Z. Phys. 94, 717 (1935).

[11] W. Shockley, Phys. Rev. 56, 317 (1939).

[12] M. W. Cole and M. H. Cohen, Phys. Rev. Lett. 23, 1238-41 (1969).

[13] M. W. Cole, Phys. Rev. B 2, 4239-52 (1970).

[14] C. C. Grimes and T. R. Brown, Phys. Rev. Lett. 32, 280-3 (1974).

[15] P. Johnson and N. Smith, Phys. Rev. B 27, 2527-30 (1983).

[16] V. Dose, W. Altmann, A. Goldmann, U. Kolac, and J. Rogozik, Phys. Rev. Lett. 52, 1919-21 (1984).

[17] D. Straub and F. Himpsel, Phys. Rev. Lett. 52, 1922-4 (1984).

[18] P. M. Echenique and J. B. Pendry, J. Phys. C: Solid State Phys. 11, 2065-75 (1978).

[19] N. V. Smith, Phys. Rev. B 32, 3549-55 (1985).

[20] C. B. Harris, N.-H. Ge, R. L. Lingle, Jr., J. D. McNeill, and C. M. Wong, Annu. Rev. Phys. Chem. 48, 711-744 (1997).

[21] J. Richard M. Osgood and X. Wang, "Image states on single-crystal Metal surfaces," in Solid State Physics, H. Ehrenreich and F. Spaepen, eds., (Academic Press, New York, 1998), Vol. 51, pp. 1-80. 
[22] W. R. Smythe, Static and Dynamic Electricity (McGraw-Hill, New York, 1950).

[23] L. D. Landau and E. M. Lifshitz, Electrodynamics of Continuous Media (Pergamon, Oxford, 1984).

[24] E. G. McRae, Rev. Mod. Phys. 51, 541-68 (1979).

[25] N. V. Smith, Rep. Prog. Phys. 51, 1227-94 (1988).

[26] P. M. Echenique and J. B. Pendry, Prog. Surf. Sci. 32, 111-172 (1990).

[27] E. G. McRae and M. L. Kane, Surf. Sci. 108, 435-45 (1981).

[28] E. W. Plummer and W. Eberhardt, Adv. Chem. Phys. 49, 533 (1982).

[29] E. T. Goodwin, Proc. Cambridge Philos. Soc. 35, 205 (1939).

[30] T. Fauster, Appl. Phys. A A59, 479-86 (1994).

[31] K. Giesen, F. Hage, F. J. Himpsel, H. J. Riess, and W. Steinmann, Phys. Rev. $B$ 35, 971-4 (1987).

[32] D. Straub and F. J. Himpsel, Phys. Rev. B 33, 2256-62 (1986).

[33] P. M. Echenique, F. Flores, and F. Sols, Phys. Rev. Lett. 55, 2348-50 (1985).

[34] M. Aeschlimann, M. Bauer, and S. Pawlik, Chem. Phys. 205, 127-141 (1996).

[35] S. Ogawa and H. Petek, Surface. Sci. 358, 585-594 (1996). 
[36] P. L. de Andrés, P. M. Echenique, and F. Flores, Phys. Rev. B 39, 10356-8 (1989).

[37] R. W. Schoenlein, J. G. Fujimoto, G. L. Eesley, and T. W. Capehart, Phys. Rev. Lett. 61, 2596-9 (1988).

[38] R. W. Schoenlein, J. G. Fujimoto, G. L. Eesley, and T. W. Capehart, Phys. Rev. B 41, 5436-9 (1990).

[39] R. W. Schoenlein, J. G. Fujimoto, G. L. Eesley, and T. W. Capehart, Phys. Rev. B 43, 4688-4698 (1991).

[40] U. Hofer, I. L. Shumay, C. Reuss, U. Thomann, W. Wallauer, and T. Fauster, Science. 277, 1480-1482 (1997).

[41] P. L. de Andrés, P. M. Echenique, and F. Flores, Phys. Rev. B 35, 4529-32 (1987).

[42] L. Hedin and S. Lundqvist, in Solid State Physics (Academic Press, New York, 1969), Vol. 23, p. 1.

[43] M. Wolf, E. Knoesel, and T. Hertel, Phys. Rev. B 54, R5295-R5298 (1996).

[44] E. V. Chulkov, I. Sarria, V. M. Silkin, J. M. Pitarke, and P. M. Echenique, Phys. Rev. Lett. 80, 4947-4950 (1998).

[45] R. L. Lingle, Jr., N.-H. Ge, R. E. Jordan, J. D. Mcneill, and C. B. Harris, Chem. Phys. 205, 191-203 (1996). 
[46] Angle-Resolved Photoemission: Theory and Current Applications, S. D. Kevan, ed., (Elsevier, Amsterdam, 1992).

[47] W. Steinmann, Appl. Phys. A A49, 365-77 (1989).

[48] N. V. Smith and S. D. Kevan, "Introduction," in Angle-Resolved Photoemission: Theory and Current Applications, S. D. Kevan, ed., (Elsevier, Amsterdam, 1992), pp. 1-14.

[49] N. W. Ashcroft and N. D. Mermin, Solid State Physics (Saunders College Publishing, New York, 1976).

[50] K. Giesen, F. Hage, F. J. Himpsel, H. J. Riess, W. Steinmann, and N. V. Smith, Phys. Rev. B 35, 975-8 (1987).

[51] S. Schuppler, N. Fischer, T. Fauster, and W. Steinmann, Phys. Rev. B 46, 13539-47 (1992).

[52] R. Fischer, S. Schuppler, N. Fischer, T. Fauster, and W. Steinmann, Phys. Rev. Lett. 70, 654-657 (1993).

[53] W. Wallauer and T. Fauster, Surf. Sci. 331-333, 731-5 (1995).

[54] J. E. Ortega, F. J. Himpsel, R. Haight, and D. R. Peale, Phys. Rev. B 49, 13859-62 (1994).

[55] W. S. Fann, R. Storz, H. W. K. Tom, and J. Bokor, Phys. Rev. Lett. 68, 2834-7 (1992). 
[56] C. A. Schmuttenmaer, M. Aeschlimann, H. E. Elsayed-Ali, R. J. D. Miller, D. A. Mantell, J. Cao, and Y. Gao, Phys. Rev. B 50, 8957-60 (1994).

[57] S. Ogawa, H. Nagano, and H. Petek, Phys. Rev. B 55, 10869-77 (1997).

[58] J. Cao, Y. Gao, R. J. D. Miller, H. E. Elsayed-Ali, and D. A. Mantell, Phys. Rev. B 56, 1099-102 (1997).

[59] R. Haight and M. Baeumler, Phys. Rev. B 46, 1543-52 (1992).

[60] A. Rettenberger and R. Haight, Phys. Rev. Lett. 76, 1912-15 (1996).

[61] C. A. Schmuttenmaer, C. C. Miller, J. W. Herman, J. Cao, D. A. Mantell, Y. Gao, and R. J. D. Miller, Chem. Phys. 205, 91-108 (1996).

[62] M. Aeschlimann, M. Bauer, S. Pawlik, W. Weber, R. Burgermeister, D. Oberli, and H. Siegmann, Phys. Rev. Lett. 79, 5158-61 (1997).

[63] H. Petek, A. P. Heberle, W. Nessler, H. Nagano, S. Kubota, S. Matsunami, N. Moriya, and S. Ogawa, Phys. Rev. Lett. 79, 4649-52 (1997).

[64] J. D. McNeill, R. L. Lingle, Jr., N.-H. Ge, C. M. Wong, R. E. Jordan, and C. B. Harris, Phys. Rev. Lett. 79, 4645-4648 (1997).

[65] C. C. Miller, S. J. Diol, C. A. Schmuttenmaer, J. Cao, D. Mantell, R. J. D. Miller, and Y. Gao, J. Phys. D, Appl. Phys. 30, 1416-20 (1997). 
[66] N.-H. Ge, C. M. Wong, R. L. Lingle, Jr., J. D. McNeill, K. J. Gaffney, and C. B. Harris, Science 279, 202-205 (1998).

[67] R. Haight, Surf. Sci. Rep. 21, 277-325 (1995).

[68] H. Petek and S. Ogawa, Prog. Surf. Sci. 56, 239-310 (1997).

[69] D. F. Padowitz, W. R. Merry, R. E. Jordan, and C. B. Harris, Phys. Rev. Lett. 69, 3583-3586 (1992).

[70] W. R. Merry, R. E. Jordan, D. F. Padowitz, and C. B. Harris, Surface. Sci. 295, 393-401 (1993).

[71] W. R. Merry, Ph.D. thesis, University of California at Berkeley, 1992.

[72] C. V. Shank, R. L. Fork, R. Yen, R. H. Stolen, and W. J. Tomlinson, Appl. Phys. Lett. 40, 761-3 (1982).

[73] B. Nikolaus and D. Grischkowsky, Appl. Phys. Lett. 42, 1-2 (1983).

[74] R. H. Stolen, C. V. Shank, and W. J. Tomlinson, "Procedure for Calculating optical pulse Compression from Fiber-grating Combinations," in Ultrafast Phenomena IV, D. H. Auston and K. B. Eisenthal, eds., (Springer-Verlag, Berlin, 1984), pp. $46-48$.

[75] W. J. Tomlinson, R. H. Stolen, and C. V. Shank, J. Opt. Soc. Am. B 1, 139-49 (1984). 
[76] S. Oda, Y. Segawa, P. H. Kim, S. Namba, and N. Kodama, Jpn. J. Appl. Phys. 2, Lett. 28, L1977-8 (1989).

[77] N. Sarukura, Y. Ishida, T. Yanagawa, and H. Nakano, Appl. Phys. Lett. 57, 229-30 (1990).

[78] N. Sarukura and Y. Ishida, IEEE J. Quantum Electron. 28, 2134-41 (1992).

[79] T. B. Norris, Opt. Lett. 17, 1009-11 (1992).

[80] M. K. Reed, M. K. Steiner-Shepard, and D. K. Negus, Opt. Lett. 19, 1855-7 (1994).

[81] R. L. Lingle, D. F. Padowitz, R. E. Jordan, J. D. Mcneill, and C. B. Harris, Phys. Rev. Lett. 72, 2243-2246 (1994).

[82] L. E. Firment and G. A. Somorjai, J. Chem. Phys. 69, 3940-52 (1978).

[83] K. Giesen, F. Hage, F. J. Himpsel, H. J. Riess, and W. Steinmann, Phys. Rev. $B$ 33, 5241-4 (1986).

[84] S. D. Kevan and R. H. Gaylord, Phys. Rev. B 36, 5809-18 (1987).

[85] N. Fischer, S. Schuppler, R. Fischer, T. Fauster, and W. Steinmann, Phys. Rev. $B$ 47, 4705-13 (1993).

[86] J. D. McNeill, R. L. Lingle, Jr., R. E. Jordan, D. F. Padowitz, and C. B. Harris, J. Chem. Phys. 105, 3883-91 (1996). 
[87] A. Goldmann, V. Dose, and G. Borstel, Phys. Rev. B 32, 1971-80 (1985).

[88] B. Reihl, R. Schlittler, and H. Neff, Phys. Rev. Lett. 52, 1826-9 (1984).

[89] W. Boeck and D. Kolb, Surf. Sci. 118, 613-22 (1982).

[90] R. Paniago, R. Matzdorf, G. Meister, and A. Goldmann, Surf. Sci. 336, 113-22 (1995).

[91] T. Hertel, E. Knoesel, M. Wolf, and G. Ertl, Phys. Rev. Lett. 76, 535-538 (1996).

[92] Z. Wu, B. Quiniou, J. Wang, and R. M. Osgood, Jr., Phys. Rev. B 45, 9406-9 (1992).

[93] E. Knoesel, A. Hotzel, and M. Wolf, J. Electron Spectrosc. Relat. Phenom. 88-9, 577-84 (1998).

[94] J. Holzl and F. K. Schulte, "Work Function of Metals," in Solid Surface Physics, Vol. 85 of Springer Tracts in Modern Physics (Springer-Verlag, Berlin, 1979).

[95] S. J. Romanowski and J. A. N. F. Gomes, J. Electroanal. Chem. 373, 133-40 (1994).

[96] P. Heimann and H. Neddermeyer, Solid State Commun. 26, 279-82 (1978).

[97] J. A. Knapp, F. J. Himpsel, A. R. Williams, and D. E. Eastman, Phys. Rev. B 19, 2844-9 (1979). 
[98] N. E. Christensen, Phys. Rev. B 20, 3205-9 (1979).

[99] A. Goldmann and R. Matzdorf, Prog. Surf. Sci. 42, 331-50 (1993).

[100] T. Fauster, Appl. Phys. A A59, 639-44 (1994).

[101] R. Fischer and T. Fauster, Phys. Rev. B 51, 7112-7115 (1995).

[102] X. Y. Wang, R. Paiella, and R. M. Osgood, Jr., Phys. Rev. B-condensed. Matter. 51, 17035-17039 (1995).

[103] F. Manghi, P. Perfetti, and B. Reihl, Solid State Commun. 76, 1371-3 (1990).

[104] J. A. Stroscio, R. M. Feenstra, and A. P. Fein, Phys. Rev. Lett. 57, 2579-82 (1986).

[105] R. L. Lingle, Jr., J. D. McNeill, R. E. Jordan, and C. B. Harris, 1994, presented at Annu. Meet. Amer. Chem. Soc., 204th, Washington, D.C.

[106] A. O. Allen, "Drift Mobilities and Conduction Band Energies of Excess Electrons in Dielectric Liquids," NBS Report No. NSRDS-NBS 58, U.S. Dept. of Commerce (1976) .

[107] M. Cole, Phys. Rev. B 3, 4418-22 (1971).

[108] D. T. Colbert and W. H. Miller, J. Chem. Phys. 96, 1982 (1992).

[109] CRC Handbook of Chemistry and Physics, 71 ed., D. R. Lide, ed., (CRC Press, Boca Raton, 1990-1991). 
[110] D. M. Small, Handbook of Lipid Research 4: The Physical Chemistry of Lipids (Plenum Press, New York, 1986).

[111] S. C. Miller, A W.K.B.-Type Approximation to the Schrodinger Equation (1953).

[112] A. K. Ghatak, Modified Airy Function and WKB Solutions to the Wave Equation (NIST, Gaithersburg, 1991).

[113] W. H. Miller, personal communication.

[114] C. Reuß, Diplomarbeit, Universität München, 1994.

[115] J. D. Jackson, private communication (unpublished).

[116] J. D. McNeill, Ph.D. thesis, University of California at Berkeley, in preparation.

[117] G. H. Wannier, Elements of Solid State Theory (University Press, Cambridge, 1959).

[118] G. Zimmerer, "Creation, Motion and Decay of Excitons in Rare-Gas Solids," in Excited-State Spectroscopy in Solids, U. Grassano and N. Terzi, eds., (NorthHolland, Amsterdam, 1987), pp. 37-102.

[119] T. Ando, A. B. Fowler, and F. Stern, Rev. Mod. Phys. 54, 437-672 (1982).

[120] P. W. Adams and M. A. Paalanen, phys. Rev. Lett. 61, 451-54 (1988).

[121] C. C. Grimes and G. Adams, Phys. Rev. Lett. 42, 795-98 (1979). 
[122] A. E. Y., Phys. Rev. Lett. 52, 1449-52 (1984).

[123] E. T. Goodwin, Proc. Cambridge Philos. Soc. 35, 221 (1939).

[124] K. S. Song and R. T. Williams, Self-Trapped Excitons (Springer-Verlag, Berlin, 1993).

[125] Z. Liu and B. Berne, J. Chem. Phys. 99, 9054-69 (1993).

[126] N.-H. Ge, C. M. Wong, and C. B. Harris, Account. Chem. Res. (1998), submitted.

[127] C. M. Wong, J. D. McNeill, K. J. Gaffney, N.-H. Ge, A. D. Miller, S. H. Liu, and C. B. Harris, J. Phys. Chem. (1998), submitted.

[128] C. M. Wong, Ph.D. thesis, University of California at Berkeley, in preparation.

[129] R. L. Lingle, D. F. Padowitz, R. E. Jordan, J. D. Mcneill, and C. B. Harris, "Localization of Electrons at Interfaces," in Reaction Dynamics in Clusters and Condensed Phases, J. Jortner, R. D. Levine, and B. Pullman, eds., (Kluwer Academic Publishers, Dordrecht, 1994), pp. 169-178.

[130] R. Fischer, T. Fauster, and W. Steinmann, Phys. Rev. B 48, 15496-15499 (1993).

[131] L. D. Landau, Phys. Z. Sowjentunion 3, 664 (1933).

[132] T. Holstein, Ann. Phys. 8, 343 (1959). 
[133] A. L. Shluger and A. M. Stoneham, J. Phys.: Condens. Matter 5, 3049-3086 (1993).

[134] W. Hayes and A. M. Stoneham, Defects and Defect Processes in Nonmetallic Solids (Wiley, New York, 1985).

[135] Desorption Induced by Electronic Transitions, A. R. Burns, E. B. Stechel, and D. R. Jennison, eds., (Springer, Berlin, 1993).

[136] J. G. Bednorz and K. A. Muller, Z. Phys. B 64, 189 (1986).

[137] Anharmonic Properties of High-Tc Cuprates, D. M. et al., ed., (World Scientific, Singapore, 1995).

[138] T. L. Gilbert, Lecture Notes for NATO Summer School in Solid State Physics, Ghent, Belgium, 1966, unpublished, as referenced in [133, 124].

[139] D. Emin and T. Holstein, Phys. Rev. Lett. 36, 323-6 (1976).

[140] Y. Toyozawa and Y. Shinozuka, J. Phys. Soc. Jpn. 48, 472-8 (1980).

[141] Y. Toyozawa, "Electrons, Holes and Excitons in Deformable Lattice," in Relaxation of Elementary Excitations, R. Kubo and E. Hanamura, eds., (SpringerVerlag, Berlin, 1980), pp. 3-18.

[142] M. Ueta, H. Kanzaki, K. Kobayashi, Y. Toyozawa, and E. Hanamura, Excitonic processes in solid (Springer-Verlag, Berlin, 1986). 
[143] H. Sumi, "Peculiarities in Exciton-polaron Formation and self-trapping in Low Dimensions," in Excitonic Processes in Condensed Matter, Proc. SPIE 2362, J. Singh, ed., (SPIE, Bellingham, 1995), pp. 108-119.

[144] R. A. Marcus and N. Sutin, Biochim. Biophys. Acta 811, 265 (1985).

[145] A. A. Stuchebrukhov and X. Y. Song, J. Chem. Phys. 101, 9354-9365 (1994).

[146] J. Ulstrup, Charge Transfer Processes in Condensed Media (Springer-Verlag, Berlin, 1979).

[147] R. A. Marcus, J. Chem. Phys. 24, 966 (1956).

[148] R. A. Marcus, Ann. Rev. Phys. Chem. 15, 155 (1964).

[149] V. G. Levich and R. R. Dogonadze, Dokl. Acad. Nauk. SSSR 124, 123 (1959).

[150] R. R. Dogonadze, A. M. Kuznetsov, and T. A. M. Marsagishvili, Electrochim. Acta 25, 1 (1980).

[151] A. Ovchinnikov and M. Ovchinnikova, Soviet Physics - Doklady 14, 447-8 (1969).

[152] L. D. Landau, Phys. Z. Sowjentunion 2, 46 (1932).

[153] C. Zenar, Proc. R. Soc. London, Ser. A 137, 696 (1932).

[154] C. Zenar, Proc. R. Soc. London, Ser. A 140, 660 (1933). 
[155] X. Y. Song and A. A. Stuchebrukhov, J. Chem. Phys. 99, 969-978 (1993).

[156] R. P. Feynman, Statistical Mechanics (Addison Wesley, Reading, 1972).

[157] R. P. Feynman and A. R. Hibbs, Quantum Mechanics and Path Integrals (McGraw-Hill, New York, 1965).

[158] G. Parisi, Statistical Field Thory (Addison Wesley, Reading, 1988).

[159] D. Chandler, "Theory of Quantum Processes in Liquids," in Les Houches, Session LI, 1989, Part I, Liquids, Freezing and Glass Transition, D. Levesque, J. P. Hansen, and J. Zinn-Justin, eds., (Elsevier, New York, 1991), pp. 193-285.

[160] R. A. Kuharski, J. S. Bader, D. Chandler, M. Sprik, M. L. Klein, and R. W. Impey, J. Chem. Phys. 89, 3248-57 (1988).

[161] J. S. Bader, R. A. Kuharski, and D. Chandler, J. Chem. Phys. 93, 230-236 (1990).

[162] J. S. Langer, Ann. Phys. 41, 108 (1967).

[163] J. S. Langer, Ann. Phys. 54, 258 (1969).

[164] C. G. Callan, Jr. and S. Coleman, Phys. Rev. D 16, 1762-8 (1977).

[165] I. Affleck, Phys. Rev. Lett. 46, 388-91 (1981).

[166] M. J. Gillan, J. Phys. C: Solid State Phys. 20, 3621-41 (1987). 
[167] A. A. Stuchebrukhov, J. Chem. Phys. 95, 4258-4276 (1991).

[168] G. A. Voth, D. Chandler, and W. H. Miller, J. Chem. Phys. 91, 7749-60 (1989).

[169] D. Chandler, Introduction to Modern Statistical Mechanics (Oxford, New York, 1987).

[170] J. N. Gehlen and D. Chandler, J. Chem. Phys. 97, 4958-4963 (1992).

[171] J. N. Gehlen, D. Chandler, H. J. Kim, and J. T. Hynes, J. Phys. Chem. 96, 1748-1753 (1992).

[172] A. J. Leggett, S. Chakravarty, A. T. Dorsey, M. P. A. Fisher, A. Garg, and W. Zwerger, Rev. Mod. Phys. 59, 1-85 (1987).

[173] X. Y. Song, private communication.

[174] G. L. Closs and J. R. Miller, Science 240, 440 (1988).

[175] S. Efrima and M. Bixon, Chem. Phys. 13, 447-60 (1976).

[176] T. Holstein, Ann. Phys. 8, 325 (1959).

[177] A. Wortman, Master's thesis, University of California at Berkeley, 1958.

[178] E. A. Silinsh and V. Čápek, Organic Molecular Crystals : Interaction, Localization, and transport phenomen (American Institute of Physics, New York, 1994). 
[179] R. G. Snyder, J. Chem. Phys. 47, 1316 (1967).

[180] A. Ovchinnikov and M. Ovchinnikova, Soviet Physics - JETP 29, 688-93 (1969).

[181] R. R. Dogonadze, A. M. Kuznetsov, M. A. Vorotyntsev, and M. G. Zaqaraia, J. Electroanal. Chem. 75, 315 (1977).

[182] R. R. Dogonadze, A. M. Kuznetsov, and M. A. Vorotyntsev, Z. Phys. Chem. $25,1(1976)$.

[183] X. Y. Song and R. A. Marcus, J. Chem. Phys. 99, 7768-7773 (1993).

[184] C. P. Hsu, X. Y. Song, and R. A. Marcus, J. Phys. Chem. B. 101, 2546-2551 (1997).

[185] C. P. Hsu, Y. Georgievskii, and R. A. Marcus, J. Phys. Chem. A. 102, 26582666 (1998).

[186] W. G. Scaife, J. K. Vij, G. Evans, and M. Evans, J. Phys. D, Appl. Phys. 15, 1279-83 (1982).

[187] J. K. Vij, IL Nuovo Cimento 2D, 751-62 (1983).

[188] X. Y. Song, D. Chandler, and R. A. Marcus, J. Phys. Chem. 100, 11954-11959 (1996).

[189] D. Chandler, lecture notes for statistical mechanics. 
[190] S. Iwai, T. Tokizaki, A. Nakamura, K. Tanimura, N. Itoh, and A. Shluger, Phys. Rev. Lett. 76, 1691-4 (1996). 